\author{
Universidade de São Paulo \\ Instituto de Física
}

\title{
Espectroscopia do Todo-Charme Tetraquark
}

\author{
Vinícius Rodrigues Debastiani
}

Orientador: Prof. Dr. Fernando Silveira Navarra

Banca Examinadora:

Prof. Dr. Fernando Silveira Navarra (IFUSP)

Prof. Dr. Fabiana Carvalho (UNIFESP)

Prof. Dr. Tobias Frederico (ITA)

Dissertação de mestrado apresentada ao Instituto de Física para a obtenção do título de Mestre em Ciências 


\section{FICHA CATALOGRÁFICA \\ Preparada pelo Serviço de Biblioteca e Informação do Instituto de Física da Universidade de São Paulo}

Debastiani, Vinícius Rodrigues

Espectroscopia do Todo-Charme Tetraquark. São Paulo, 2016.

Dissertação (Mestrado) - Universidade de São Paulo. Instituto de Física. Depto. Física Experimental.

Orientador: Prof. Dr. Fernando Silveira Navarra

Área de Concentração: Física.

Unitermos: 1. Tetraquark; 2. Equação de Schrodinger; 3. Partículas (Física Nuclear); 4. Quark; 5. Spin.

USP/IF/SBI-039/2016 


\title{
University of Sao Paulo \\ Institute of Physics
}

\section{Spectroscopy of the All-Charm Tetraquark}

\author{
Vinícius Rodrigues Debastiani
}

Supervisor: Prof. Dr. Fernando Silveira Navarra

Master's dissertation submitted to the Institute of Physics in partial fulfillment of the requirements for the degree of Master of Science

Evaluation committee:

Prof. Dr. Fernando Silveira Navarra (IFUSP)

Prof. Dr. Fabiana Carvalho (UNIFESP)

Prof. Dr. Tobias Frederico (ITA) 


\section{Acknowledgements}

We would like to thank professor F. F. Schöberl for the Mathematica notebook used in the numerical calculations. Also, we would like to thank the support and the interesting discussions with professors Fernando S. Navarra, Eulogio B. Oset, Manoel R. Robilotta, Alessio Mangiarotti, Marina Nielsen, Alberto Martínez Torres, Kanchan Khemchandani, Renato Higa, Walter A. de S. Pedra, Sylvio R. A. Canuto, Juan M. Nieves Pamplona, Manoel J. Vicente Vacas and many others.

The discussions with many friends were also important and I would like to thank Fábio Chibana, Guilherme R. Germano, Lucas M. Cornetta, Leandro Milhomens da Fonseca, Bruno D. da Silva Moreira, David A. Fogaça, Samuel M. Sanches Júnior, Luiz P. de Oliveira, Andre V. Giannini, Diego Spiering Pires, Jorgivan M. Dias, among many others from the Instituto de Física of Universidade de São Paulo (IFUSP) and also Pedro Fernandez Soler, Astrid N. Hiller Blin, David Eduardo Saul Sala, Francesca Aceti, Miguel Albaladejo Serrano, Javier Fuentes Martin, among many others from the Instituto de Física Corpuscular of Universitat de València (IFIC-UV/CSIC).

Finally, none of this would be possible without the support of my parents Wladimir and Cássia, and my sister Bruna, all of which I wish to specially thank.

During this work the student received financial support from the Conselho Nacional de Desenvolvimento Científico e Tecnológico (CNPq) (Brazil) and from Generalitat Valenciana in the Programa Santiago Grisolía (Spain). 


\section{Resumo}

\section{DEBASTIANI, V. R. Espectroscopia do Todo-Charme Tetraquark.}

Dissertação de Mestrado - Instituto de Física, Universidade de São Paulo, São Paulo, Brasil, 2016.

Introduzimos um método não-relativístico para estudar a espectroscopia de estados ligados hadrônicos compostos por quatro quarks charme, na figura de diquark-antidiquark. Resolvendo numericamente a equação de Schrödinger com dois potenciais diferentes inspirados no potencial de Cornell, de uma maneira semelhante aos modelos de quarkonium pesado para mésons, nós fatoramos o problema de 4 corpos em três sistemas de 2 corpos: primeiro o diquark e o antidiquark, que são compostos por dois quarks (antiquarks) em um estado de antitripleto de cor. No próximo passo eles são considerados como os blocos para construir o tetraquark, onde a sua interação leva a um singleto de cor. Termos dependentes de spin (spin-spin, spin-órbita e tensor) são usados para descrever o desdobramento do espectro e a separação entre estados com diferentes números quânticos. Atenção especial é dada à interação do tensor entre duas partículas de spin 1, com uma discussão detalhada da estratégia adotada. A interação spin-spin é tratada perturbativamente no primeiro modelo e incluída no potencial de ordem zero no segundo. A contribuição de cada termo de interação também é analisada e comparada. Dados experimentais recentes de estados bem estabelecidos de mésons de charmonium são utilizados para fixar os parâmetros de ambos os modelos (em um procedimento de ajuste minimizando $\chi^{2}$ ), obtendo uma reprodução satisfatória do espectro do charmonium. As diferenças entre modelos são discutidas no contexto do charmonium, diquarks e tetraquarks. Nós concluímos que quase todas as ondas $\mathrm{S}$ e $\mathrm{P}$ (e as respectivas primeiras excitações radiais) do todo-charme tetraquark composto por diquarks de spin 1 estão entre 5.8 e $7 \mathrm{GeV}$, acima do limite de dissociação espontânea em pares de charmonium de baixa energia como dois $\eta_{c}$ ou $J / \psi$, o que sugere que esses poderiam ser os canais ideais para procurar por esses estados, e desenvolver o atual conhecimento de estados multiquarks.

Palavras-Chave: Tetraquark, Equação de Schrödinger, Partículas (Física Nuclear), Quark, Spin. 


\section{Abstract}

\section{DEBASTIANI, V. R. Spectroscopy of the All-Charm Tetraquark.}

Master's Dissertation - Institute of Physics, University of Sao Paulo, Sao Paulo, Brazil, 2016.

We introduce a non-relativistic framework to study the spectroscopy of hadronic bound states composed of four charm quarks in the diquark-antidiquark picture. By numerically solving the Schrödinger equation with two different Cornell-inspired potentials in a similar way of heavy quarkonium models of mesons, we factorize the 4-body problem into three 2-body systems: first the diquark and the antidiquark, which are composed of 2 quarks (antiquarks) into a color antitriplet state. In the next step they are considered as the tetraquark building blocks, where their interaction leads to a color singlet. Spin-dependent terms (spin-spin, spin-orbit and tensor) are used to describe the splitting structure of the spectrum and account for different quantum numbers of each state. Special attention is given to the tensor interaction between two particles of spin 1, with a detailed discussion of the adopted strategy. The spin-spin interaction is addressed perturbatively in the first model and included in the zeroth-order potential in the second one. The contribution of each interaction term is also analysed and compared. Recent experimental data of reasonably well-established charmonium mesons are used to fix the parameters of both models (with a fitting procedure minimizing $\chi^{2}$ ), obtaining a satisfactory reproduction of charmonium spectrum. The differences between models are discussed in the charmonium, diquark and tetraquark context. We conclude that almost all the $\mathrm{S}$ and $\mathrm{P}$ waves (and respective first radial excitations), of the all-charm tetraquark composed by spin 1 diquarks are in the range between 5.8 to $7 \mathrm{GeV}$, above the threshold of spontaneous decay in low-lying charmonium pairs, like two $\eta_{c}$ or $J / \psi$, what suggests that this could be the ideal channels to look for these states, and develop the current understanding of multiquark states.

Keywords: Tetraquark, Schrödinger Equation, Particles (Nuclear Physics), Quark, Spin. 
x ABSTRACT 


\section{Contents}

$\begin{array}{ll}\text { Abstract } & \text { ix }\end{array}$

Contents $\quad$ xii

Preface $\quad$ xiii

1 Introduction $\quad 1$

1.1 QCD, Quarks and Hadrons . . . . . . . . . . . . . . . . . . . 1

1.2 Exotics . . . . . . . . . . . . . . . . . . . 3

1.3 The All-Charm Tetraquark . . . . . . . . . . . . . . . . . . . . 4

2 The Tetraquark Model $\quad 9$

2.1 The Non-Relativistic Framework . . . . . . . . . . . . . . . . . . . . . . . . 10

2.2 Constituents Velocities . . . . . . . . . . . . . . . . . . 12

2.3 The Hamiltonian . . . . . . . . . . . . . . . . . . . . . . . . . . . . . . 12

2.3.1 Model 1 . . . . . . . . . . . . . . . . . . 13

2.3 .2 Model $2 \ldots \ldots \ldots \ldots \ldots$

2.4 Color Factor $\kappa_{s} \ldots \ldots \ldots \ldots \ldots \ldots \ldots$

2.4 Quark - Antiquark . . . . . . . . . . . . . 15

2.4.2 Quark-Quark .................... 21

2.5 The Coupling Constant $\alpha_{s} \ldots \ldots \ldots \ldots \ldots$. . . . . . . . . . . 24

2.6 Wavefunction at the Origin . . . . . . . . . . . . . 25

2.6 .1 Generic $|\Psi(0)|^{2} \ldots \ldots \ldots$

$2.6 .2 \quad$ S-wave $|\Psi(0)|^{2} \ldots \ldots \ldots \ldots \ldots$

2.6.3 Discussion for $|\Psi(0)|^{2} \ldots \ldots \ldots \ldots \ldots$. . . . . . . . . . . 27

2.7 The Tetraquark Wavefunction . . . . . . . . . . . . . . . . . . 28

2.7.1 Angular Momentum Coupling . . . . . . . . . . . . . . . . . 28

2.7.2 $J^{P C}$ Quantum Numbers in the Tetraquark . . . . . . . . . . . . 32

3 Spin-Dependent Interactions 35

3.1 Spin-Spin . . . . . . . . . . . . . . . . . . . . 36

3.2 Spin-Orbit . . . . . . . . . . . . . . . . . 37

3.3 Tensor . . . . . . . . . . . . . . . . . . . . . 38 
3.3.1 Two spin $1 / 2$ particles . . . . . . . . . . . . . . . . 40

3.3 .2 Two spin 1 particles . . . . . . . . . . . . . . . . . . 41

3.4 The Spin-Average . . . . . . . . . . . . . . . . . . 55

$\begin{array}{lll}4 & \text { Mesons } & 57\end{array}$

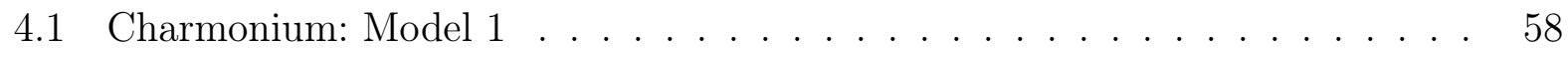

4.1.1 Model 1: Fit with 13 input . . . . . . . . . . . . . . 59

4.1.2 Model 1 with constant $V_{0}$ : Fit with 13 input . . . . . . . . . 63

4.1.3 Model 1: Fit with 6 input $(1 \mathrm{~S}$ and $1 \mathrm{P}) \ldots \ldots . \ldots . \ldots 6$

4.1.4 Model 1 with modified Spin-Orbit: Fit with 6 input (1S and 1P) . . . 64

4.1.5 Model 1: Fit with 11 input for comparison with model $2 \ldots 6$

4.2 Charmonium: Model $2 \ldots \ldots \ldots$. . . . . . . . . . . . . 68

4.2.1 Model 2: Fit with 13 input . . . . . . . . . . . . . . . 69

4.2.2 Model 2: Fit with 11 input for comparison with model 1 . . . . . . 73

4.3 Charmonium: Comparison with Experimental Data . . . . . . . . . . . . 74

$\begin{array}{lll}5 & \text { Diquarks } & 77\end{array}$

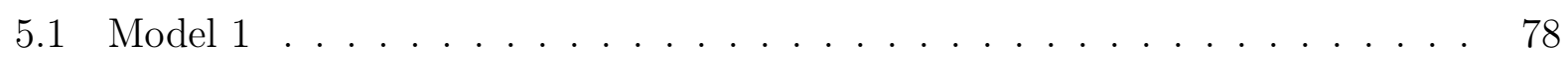

5.2 Model 1 with modified Spin-Orbit . . . . . . . . . . . . . . 80

5.3 Model $2 \ldots \ldots \ldots \ldots$. . . . . . . . . . . . . . . . . . . . . . . . .

5.4 Comparisons with the Literature . . . . . . . . . . . . . . 82

6 The All-Charm Tetraquark $\quad 85$

6.1 Model $1 \ldots \ldots \ldots$. . . . . . . . . . . . . . . . . 85

6.2 Model 1 with modified Spin-Orbit . . . . . . . . . . . . . 88

6.3 Model $2 \ldots \ldots \ldots$. . . . . . . . . . . . . . . . . . . 90

6.4 Comparisons with the Literature . . . . . . . . . . . . . . . . 93

$\begin{array}{ll}\text { Conclusion } & 95\end{array}$

$\begin{array}{ll}\text { A Extra Tables: Charmonium } & 97\end{array}$

A.1 Model 1: FIT with 11 input . . . . . . . . . . . . . . . . . 97

A.2 Model 2: FIT with 11 input . . . . . . . . . . . . . . . 99

$\begin{array}{ll}\text { Bibliography } & 101\end{array}$ 


\section{Preface}

The present work is organized as follows:

Chapter 1 presents some general ideas about the theoretical scenario involved in the description of bound states of quarks, with special attention to the subject of exotic hadrons, which have been object of increasingly efforts both in experimental and theoretical works. A discussion about the history of the all-charm tetraquark in literature is presented with the motivations for its study.

Chapter 2 introduces the essence of the theoretical framework presenting the two models used to describe charmonium mesons and the tetraquark as a diquark-antidiquark bound state.

Chapter 3 presents in detail the spin interactions and the method used to calculate their contributions. Special attention is given to the coupling of angular momentum and the problem of calculating the tensor interaction between two spin 1 particles.

Chapter 4 presents the application of both models to the charmonium mesons $c \bar{c}$, where a fitting procedure is performed with recent experimental data in order to obtain the best set of parameters to reproduce it. The method is discussed together with the comparison between models.

Chapter 5 presents the results and discussions about diquarks composed of two charm quarks, using the adaptation to color antitriplets of the parameters obtained from the fit of both models to charmonium mesons. A brief comparison with other works is included.

Chapter 6 presents the results for the all-charm tetraquark $[c c][\bar{c} \bar{c}]$ using the previous results for the diquarks and the parameters from charmonium, with both models. A brief comparison with other works is included too.

Finally we draw the conclusions, discussing the strong and weak points of the adopted models, the approximations employed, the relation of our estimates with experimental data, the possible improvements and extensions of the present work, and the alternatives in the modeling of tetraquarks. 


\section{Chapter 1}

\section{Introduction}

\subsection{QCD, Quarks and Hadrons}

The current understanding of nature in Physics comprises four different types of forces [1]: the Electromagnetic, the Weak, the Strong and the Gravitational. The first two can be unified into what is called the Electroweak force, which together with the strong force and the theory of Elementary Particles compose the Standard Model, that accounts for most (but not all) of our comprehension of what the universe is made of, and which are the laws that govern the interaction of its constituents. Gravity is still an outsider in the Standard Model, and there are many different attempts to unify all the forces in a great theory of everything. Many other unresolved questions, like Dark Matter in the Cosmology subjects, or the compatibility of General Relativity and Quantum Mechanics remain as large investigation fields.

When dealing with particles interactions, we usually encounter situations where very small scales and relativistic velocities are involved. Therefore, Quantum Mechanics and Special Relativity play an important role in this context. The general tool to describe phenomena in these conditions is Quantum Field Theory, which accounts for the quantization of particles and fields. The quantization of the interactions themselves, by quantizing the fields, is usually called Second Quantization. To illustrate that, we can think about the Electromagnetic force: in "Classical" Quantum Mechanics, the interaction of the electron with the proton in the Hydrogen atom is well described with a "classical" electromagnetic field, that can be put in the form of a static potential into the Schrödinger equation to solve for the quantized bound state. Going a little further, we can obtain a better description using Relativistic Quantum Mechanics, with the Dirac equation instead of the Schrödinger one, but still using a "classical" static potential. But if we go even further and quantize the electromagnetic field, the interaction can be described by the exchange of photons, the quanta of the electromagnetic interaction, in a completely relativistic formalism. That approach was beautifully organized in what is called Quantum ElectroDynamics, or QED, one of the most successful theories in Physics, which has predicted and experimentally confirmed phenomena with very high accuracy, (the Lamb Shift or Casimir effect, for example).

Also in the context of Quantum Field Theory, we have a theory to describe the strong interaction, which is called Quantum ChromoDynamics, or QCD. It can describe interactions between quarks, elementary particles that carry color charges and are the basic constituents, for example, of the protons and neutrons that form the nuclei of atoms. The interaction between nucleons is also related to the strong force at larger scales. 
In the Standard Model the mediators of the interactions are called Gauge Bosons: the photons are responsible for the electromagnetic interaction, the $\mathrm{Z}$ and $\mathrm{W}$ bosons for the weak interaction, and the gluons for the strong interaction. Bosons have integer spins, and obey the Bose-Einstein statistics. Recently, the existence of the Higgs boson was confirmed by LHC experiments. This boson is related with the Higgs Field and a process that gives mass for elementary particles.

Apart from bosons, the elementary particles are fermions, which mean they have spin semi-integer and therefore obey the Fermi-Dirac statistics. Leptons are elementary particles that interact only through electroweak interactions: we have the electron, muon and tau, in increasingly mass order, each one with its respective neutrino. More specifically, electron, muon, and tau have mass and electric charges, while neutrinos barely have mass and no electric charges; they are directly related to weak interaction and decays. Quarks are elementary particles that carry color charges, electric charges (which are fractions of the elementary charge of the electron) and flavor. In order of increasing mass, we have six flavors of quarks: up and down $(u$ and $d)$, strange $(s)$, charm $(c)$, bottom $(b)$ and top $(t)$.

Bound states of quarks are called Hadrons, where quarks are kept together by strong interaction. There are two basic structures of hadrons: the mesons, which are bound states of a quark and an antiquark and the baryons, which are bound states of three quarks (or three antiquarks for an antibaryon). These constituents quarks are actually called the valence quarks. Protons and neutrons are examples of baryons, while interesting examples of mesons are the pions $\pi$, (discovered in the study of cosmic rays, which had a important role if the development of Nuclear Physics with the works of Yukawa), and the $J / \psi$, a charmonium meson $c \bar{c}$, which was very important in the consolidation of the quark model.

There is a difference between the force inside hadrons and between hadrons. When talking about the interaction between quarks due to the color charges and exchange of gluons, we are talking about the fundamental strong interaction. But there is also a residual strong interaction that accounts for the interaction between protons and neutrons bound as a nucleus, for example. This interaction is usually described by the exchange of mesons, not gluons, the most common case is the exchange of pions.

An important property of QCD related to color charges is the color confinement. There are three color charges (and the respective anticolors): red, green, and blue. Observables are always in the color singlet state, that is like a "white", or "blank" color, which can be imagined (in a pictorial way!) as the same amount of color and anticolor, for example in a meson composed of red and antired color; or in a baryon composed of the three colors together: red, green and blue, which also results in "white". So quarks can never be seen alone, because they carry color charges that must be confined. (We will discuss color states in detail in Section 2.4).

Gluons are the mediators of the strong interactions and they are massless vector bosons (spin 1). They carry no electric charge, only color and anticolor charges. The 3 colors can combine with 3 anticolors into an octet and a singlet. But the $S U(3)$ color symmetry of QCD doesn't allow the existence of the color singlet gluon. So we have only 8 color types of gluons, each one with a color configuration from the color octet. It is important to see some of the crucial differences between QCD and QED. The mediators of the electromagnetic interactions, the photons, carry no electric charge, so they can't interact with themselves, they only mediate the interaction between objects that actually carry electric charges, like electrons. Since gluons do carry color charges, besides mediating the interaction of particles that carry color charges, like quarks, gluons can interact with other gluons. And also because gluons carry color charge, sometimes the color of interacting particles can change in a QCD vertex, once it can be "carried" by the gluon to the other particle; the analogue can't happen 
in QED because the photon can't carry electric charges. The number of possibilities in QCD is much bigger than in QED; these examples give us some ideas to appreciate the complexity of Quantum Chromodynamics.

Another important feature of QCD is the asymptotic freedom. The general idea of asymptotic freedom relies on the property that the coupling constant of strong interactions decreases as the scale of energy increases, or similarly, as the scale of length decreases. That allows a perturbative treatment of QCD to describe many process.

In QED, the perturbative treatment of interactions can be understood by the series expansion of the amplitude of interaction in terms of the fine structure constant $\alpha$. This way one can rely on the lowest-order Feynman diagrams to study the main effect of a certain process. Higher order diagrams can be included, revealing new properties of the interaction, but their contribution is expected to decrease as the order increases. The technical details of these calculations are in the scope of Quantum Field Theory and Renormalization techniques that need to be applied in order to deal with the divergent behaviour of such amplitudes. In this work we shall not discuss in such terms, but only to show some results and qualitatively justify their employment in quarkonium models.

The inclusion of the one-loop diagram in electron-muon scattering for example, (when from the virtual exchanged photon an electron-positron pair is created and annihilated back to a photon), leads to the running coupling constant, which is a function of the exchanged momentum $\left|q^{2}\right|$. By adding up higher order diagrams, with one, two, three, ..., infinity number of loops we find the geometric series that can be explicitly summed and leads to a good approximation of the behaviour of the electromagnetic running coupling constant $\alpha_{e}\left(\left|q^{2}\right|\right)$, which is relatively constant at low momentum exchange, but increases with highly relativistic process, where $\left|q^{2}\right|$ is large. This shows that the vacuum acts like a dielectric medium, screening the electric charge. When high momentum exchange is involved, the charges get closer, and the screening effect is lower. This property is sometimes called vacuum polarization.

In QCD we have a similar phenomena: quark-antiquark loops are created from the virtual gluon exchanged. But since gluons carry color charge as well, new higher order diagrams must be included, as gluon pair loops or even gluon vertices. It turns out that the quark-antiquark loops lead to color charge confinement for small $\left|q^{2}\right|$, and the gluon corrections leads to asymptotic freedom in highly relativistic process (large $\left|q^{2}\right|$ ). The strong force running coupling constant $\alpha_{s}\left(\left|q^{2}\right|\right)$ from QCD increases for long distances and decreases for small distances. Because of that we can treat highly energetic process of QCD in a perturbative fashion with lowest-order Feynman diagrams.

\subsection{Exotics}

Apart from $q \bar{q}$ mesons and $q q q$ baryons, others structures are compatible with the QCD framework. Multiquark states with four or more quarks have already been suggested decades ago $[2,3,4]$. At first, the discussions on the tetraquark configurations were based on the MIT bag model, considering only light states. Later on, the tetraquark picture was extended to other quark models and heavy quarks were included $[5,6]$.

From the point of view of effective or residual interactions, one can simply imagine that a state of four quarks can be described through the interaction of two mesons $q \bar{q}-q \bar{q}$, five quarks states through the interaction of a meson and a baryon $q \bar{q}-q q q$ and six quarks states as the interaction of two baryons $q q q-q q q$ (two nucleons for instance, like the deuteron), and so on. 
But from the point of view of fundamental interactions (with quarks as degrees of freedom and bound states described with exchange of color objects), there are still other compatible multiquark structures, one of them is our object of study: a compact tetraquark $[q q][\bar{q} \bar{q}]$ whose constituents are diquarks, not mesons. These diquarks are assumed to be in a non-singlet color state, and therefore the diquark-antidiquark structure is expected to be a strongly bound state due to gluon exchange, in contrast with the meson molecule, which is described as a loosely bound state of two mesons (which are color singlets, and might interact through pion exchange, for example). Many other structures have been suggested in the literature, like hybrid states with gluon field excitations as degrees of freedom, among many others.

This discussion was revived in the past decade due to new experimental data that strongly suggest the existence of these multiquark states. That is because these new measured states present quantum numbers, masses and decay channels and widths that can not be explained with the regular mesons or baryons models, (therefore they are called Exotics), but it seems to be possible to treat explain many of these states with models of diquark-antidiquark tetraquarks or meson molecules. Nowadays, the existence of exotic hadrons has already been established $[7,8]$, since some of these new states were even found to be charged, what reinforces the exotic idea $[9,10]$.

In the charmonium context $[11,12]$, many of theses states are produced in B-meson factories. These machines are $e^{+} e^{-}$colliders which operates on a center of mass energy of $10.600 \mathrm{MeV}$, just enough to produce $B$ meson pairs, whose decay produces charmonium. Working in different B factories, the BABAR, BELLE and BESIII collaborations produced a large amount of data on charmonium spectra. Since 2003, new charmonium states have been discovered. Some of these states, like the $X(3943)$, can be explained as regular $c \bar{c}$ mesons, but many others, as the $X(3872)$, do not fit in the predictions of the conventional $c \bar{c}$ spectrum. The discovery of the $X(3872)$ was very important to bring back the discussion on multiquark states [13]. The majority of the models proposed to explain these new charmonium states consider them as four quarks in the charm sector $(c q \bar{c} \bar{q}, q \in u, d, s)$.

In the meson molecule approach some important references are $[14,15,16,17,18,19,20$, 21]. The alternative model of four quarks bound in a compact tetraquark was also estimulated by the new discoveries on charmonium [22]. In particular, the diquark-antidiquark picture gained lots of attention in the works of Maiani et al. [23, 24, 25, 26, 27].

The approach to exotics with QCD Sum Rules is also common, for example in Ref. [28] tetraquarks containing bottom quarks are also considered, and in Ref. [29] a tetraquark with double open charm is discussed.

\subsection{The All-Charm Tetraquark}

We have chosen to focus on tetraquarks composed of a single flavor (only charm quarks) using a diquark-antidiquark model $[c c][\bar{c} \bar{c}]$, which we will denote by $T_{4 c}$ or "The All-Charm Tetraquark". This configuration introduces additional statistical restrictions, which decreases the number of possibilities in quantum numbers, and allows one to avoid algebraic complications or inaccurate oversimplifications of the interactions. Also, all-heavy tetraquarks aren't investigated as much as the heavy-light proposals for the X, Y, Z exotics; therefore giving them special attention might introduce a bit of originality.

There are other reasons to focus in the diquark-antidiquark model. For instance, most of the experimental information on exotic candidates accumulated since the discovery of the $X(3872)$ in 2003 comes from B decays, where the molecular and tetraquark pictures still have a balanced competition. But the recent and forthcoming production of exotics in proton 
proton collisions is a promising testing ground for the diquark-antidiquark structure, because it is extremely difficult to produce molecules in this type of reaction, as it has been shown in Ref. [8]. Also, it is possible to produce four charm quarks in the high energy collisions happening, for example, at LHC.

The first discussions on the all-charm tetraquark appeared in 1975, by Iwasaky [30, 31]. Next, Chao discussed the $T_{4 c}$ in the diquark-antidiquark picture with orbital excitations and its possible production in $e^{+} e^{-}$annihilation [32].

Later, a variational method with Gaussian trial wavefunctions was employed to study narrow tetraquarks in Ref. [4], including the all-charm, using a 4-body coordinate system, assuming 2-body forces with potentials describing the exchange of color octets, and nonstable $c \bar{c} c \bar{c}$ states were obtained. Similar variational approaches, with quasi-logarithmic potentials [33], and another inspired in mesons and baryons wavefunctions [34], also studied four-quark systems with charm in the following years. The MIT bag model was also used to derive potentials for these multiquarks states, and a possible bound state of four charm quarks was obtained in the Born-Oppenheimer approximation. However, a similar work with the MIT bag and better treatment of the kinetic energy, improving the B.-O. approximation, found that the same state would be non-stable [35, 36].

A purely chromomagnetic model (where only a constant hyperfine potential acts without radial dependence) was employed in Ref. [37] to study the all-heavy tetraquark, but it also didn't lead to bound states. Another variational calculation, now using harmonic oscillator basis and the Bhaduri potential [38, 39, 40] didn't favor the existence of $Q \bar{Q} Q \bar{Q}$ bound states.

The harmonic oscillator basis was also used in a more recent study specifically about the all-charm tetraquark by Lloyd and Vary [41]. They used a non-relativistic Hamiltonian in a 4-body fashion inspired by the one gluon exchange interaction. The diagonalization procedure leads to several close-lying bound states, predicting masses around $6 \mathrm{GeV}$ for deeply bound states.

This is a typical value also encountered in Ref. [42] using hyperspherical harmonic formalism. They found possible bound states with masses around 6.515, 6.648, and 6.216 $\mathrm{GeV}$ and quantum numbers $0^{+-}$(exotic), $2^{+-}$(exotic) and $2^{++}$(nonexotic), respectively. The first and the last are found to be bound independently of the mass of the quark (bound for light quarks as well), while the $2^{+-}$and also another with $1^{+-}$are attractive only for quarks with larger mass. Since the states with exotic quantum numbers are below their corresponding thresholds (of decay in charmonium mesons pairs), they should have narrow widths. So if they are produced, the detection should be easy.

Lower mass values were obtained in Ref. [43], where the Bethe-Salpeter approach was employed and the $T_{4 c}$ with $J^{P C}=0^{++}$was found to have the mass $M_{T_{4 c}}=5.3 \pm 0.5 \mathrm{GeV}$, with dominant meson molecule component. It is even lower than the $\left(\eta_{c}\right)\left(\eta_{c}\right)$ threshold around $5.97 \mathrm{GeV}$, then the decay width may be small, since decay channels into D mesons and pairs of light mesons would necessarily involve internal gluon lines.

A lattice QCD study of the $Y$ (4260) [44] also investigated the $T_{4 c}$ and found states with $J^{P C}=1^{--}$both in meson molecule and diquark-antidiquark picture with masses $6.411 \mathrm{GeV}$ and $6.420 \mathrm{GeV}$, respectively. More recently, the lattice calculations of Refs. [45, 46] seem to disfavor the deeply bound $c \bar{c} c \bar{c}$ configuration, favoring the existence of a loosely bound $\left(\eta_{c}\right)\left(\eta_{c}\right)$ molecule.

The production of the $T_{4 c}$ was studied with single parton scattering in Refs. [47, 48], where the masses of the three S-wave states in the diquark-antidiquark picture were also estimated, resulting in 5.966, 6.051 and $6.223 \mathrm{GeV}$ for the $J^{P C}$ quantum numbers $0^{++}, 1^{+-}$ 
and $2^{++}$, respectively. In a very recent work (2016), the production of the $T_{4 c}$ was studied with double parton scattering using a version of the color evaporation model [49].

Also in 2016, tetraquarks composed only of heavy flavor charm and bottom were studied with color-magnetic model using the diquark-antidiquark picture [50], obtaining masses estimates for the S-wave tetraquarks with two methods: using effective constituents masses, resulting in masses below $6 \mathrm{GeV}$ for the $T_{4 c}$, and using meson pairs thresholds, resulting in masses above $6 \mathrm{GeV}$ for the $T_{4 c}$.

Another study from 2016 investigated the all-charm and all-bottom tetraquarks in the diquark-antidiquark picture using Moment Method in Sum Rule QCD [51], what once more reinforces the increasing interest on these multiquark configurations with four heavy quarks. As pointed in Ref. [51], the increase in energy of colliders makes it possible to produce two charmonium pairs where this all-charm tetraquark can arise. Their method predicts all masses of the $T_{4 c}$ above $6 \mathrm{GeV}$, and that most of these states are above their corresponding spontaneous decay threshold into two charmonium mesons, like $\eta_{c} \eta_{c}$ or $J / \psi J / \psi$, so these would be the ideal kind of channel to look for these states.

The majority of predictions for the $T_{4 c}$ have masses around $6 \mathrm{GeV}$, therefore lay well above the charmonium experimentally known range (which is concentrated within 3-4.5 $\mathrm{GeV}$ ). This energy gap makes the all-charm tetraquark an interesting probe for the search of exotics multiquarks, one more reason to focus in this particular tetraquark. The heavy-light tetraquark candidates in contrast (the $\mathrm{X}, \mathrm{Y}, \mathrm{Z}$ with charm quarks), are in the same mass range of conventional charmonium, what can be misleading in some cases, like the also very recent discussion of the $X(3915)$, which was assigned as the $\chi_{c 0}(2 P)(J=0)$ conventional charmonium by the Particle Data Group in 2014 [52], but recovered its position of exotic right back in $2015[53,54,55]$. However, the recently measured $\chi_{c 2}(2 P)(J=2)$ seems to be a conventional $c \bar{c}$ state (see Ref. [56] and references therein).

The search for exotic multiquark states contributes for our comprehension of role of the strong interaction in these nonconventional structures of matter. The all-charm tetraquark brings new opportunities to investigate in this subject.

A pictorial representation of the all-charm tetraquark in the diquark-antiquark scheme of our model can be seen in Figure 1.1. 


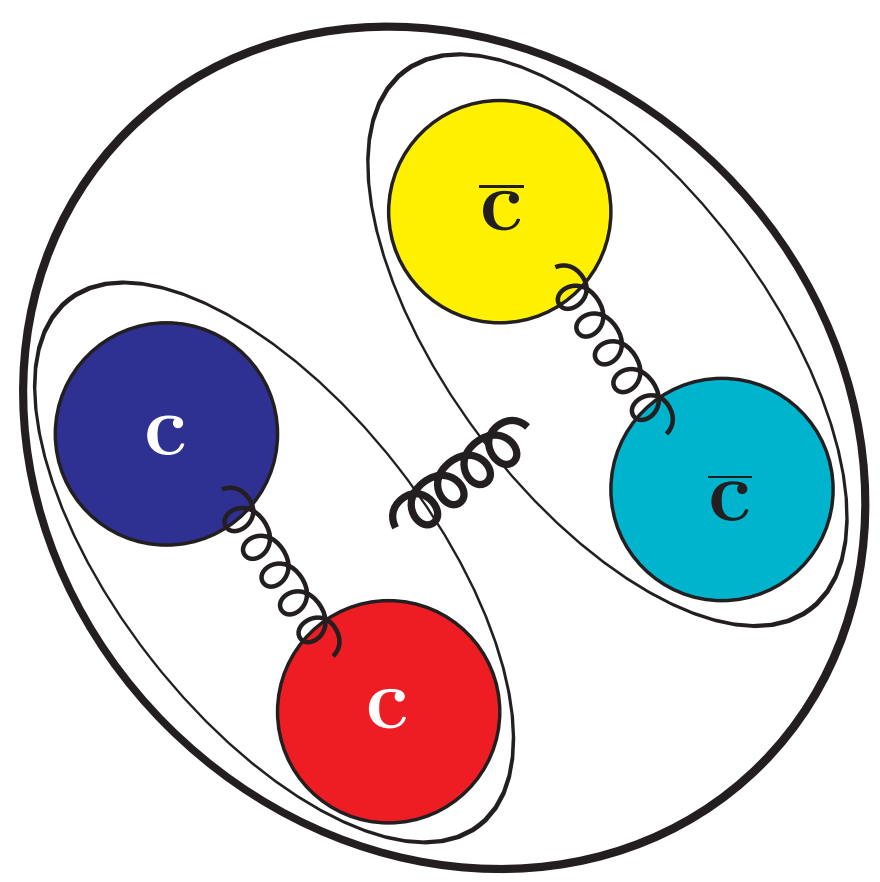

Figure 1.1: Pictorial representation of the all-charm tetraquark in the diquarkantiquark scheme. 


\section{Chapter 2}

\section{The Tetraquark Model}

When aiming at heavy tetraquark models, quark-antiquark mesons are of particular interest because they can be very similar to quark-quark states that compose the diquark, and also very similar to the diquark-antidiquark system if the later is considered as a 2-body problem to build the tetraquark.

Accordingly, in this chapter two versions of a non-relativistic potential model will be introduced following popular approaches on heavy quarkonium, similar to the ones that have been successfully employed to describe the spectrum of charmonium and bottomonium states since the 70's, like the Cornell works [57, 58], for example.

The main approximation of this tetraquark model is the factorization of the 4-body problem into three subsequent 2-body problems, with quark-quark bound as a diquark, antiquark-antiquark bound as an antidiquark, and finally diquark-antidiquark bound as the tetraquark. The inspiration of this factorization is the color structure behind it. The $S U(3)$ color symmetry of QCD allows quarks to form diquarks in an attractive color structure, and similarly the antiquarks can form an antidiquark in an attractive conjugate color structure. Then the diquark and antidiquark can form a color singlet bound state (that is also attractive), just like quark and antiquark can bound as a meson. In this way, the final tetraquark state respects the color confinement of QCD, and could in principle exist. Other color combinations and mixtures could be considered, but in the present model only the main contribution is taken into account in each step.

The presence of heavy flavor allows the perturbative treatment of QCD, considering only the lowest-order Feynman diagram in the constituents interactions. One of the most common functional form of the potentials employed in heavy quarkonium spectroscopy is the Coulomb plus linear, where the Coulomb term arises from a Lorentz vector exchange (essentially one gluon exchange) and the linear part responsible for confinement is usually associated with a Lorentz scalar exchange. Spin-dependent terms (spin-spin, spin-orbit and tensor) are included as relativistic corrections of these interactions, and they account for the splitting between states with different quantum numbers. In the first model we treat the three spin-dependent terms as perturbative corrections, causing only mass shifts, while in the second version we include the spin-spin interaction in the non-perturbative potential, what causes a slight difference between wavefunctions of states with different total spin, while the orbital corrections are still treated with perturbation theory.

A comprehensive deduction of the main aspects of the quarkonium models employed here can be found in Ref. [59], and for further discussions one could check Ref. [60]. 


\subsection{The Non-Relativistic Framework}

Usually, in heavy quark bound states the kinetic energy of the constituents is small if compared to their rest energy, hence a non-relativistic approach with static potentials can be a reasonable approximation. The procedure begins by solving the time independent Schrödinger equation to obtain the binding energy and wavefunction of each particular state:

$$
H \Psi=E \Psi \quad \longrightarrow \quad\left(T+V^{(0)}\right) \Psi=E \Psi
$$

where $T$ is the non-relativistic kinetic energy and $V^{(0)}$ is the (zeroth-order) non-perturbative potential.

It is convenient to work in the center of mass frame $(\mathrm{CM})$, because we are going to adopt a central potential (radially symmetric), and therefore we can use spherical coordinates to separate the radial and angular parts of the wavefunction.

Then our first goal is to solve radial equation, which can be written in function of only one radial coordinate $r$ that describes the relative distance separating the two particles.

The kinetic energy is written in terms of the relative momentum $\mathbf{p}$ and the reduced mass $\mu$ :

$$
T=\frac{\mathbf{p}^{2}}{2 \mu}, \quad \mu=\frac{m_{1} m_{2}}{m_{1}+m_{2}}=\frac{m}{2}, \quad \text { for } \quad m_{1}=m_{2}=m
$$

While the radial wavefunction $R(r)$ is easier to handle if we put it in its reduced form $y(r)$, with the following normalization:

$$
y(r)=r R(r), \quad \int_{0}^{\infty} r^{2} d r|R(r)|^{2}=\int_{0}^{\infty} d r|y(r)|^{2}=1
$$

So with this considerations, the canonical substitution $\mathbf{p} \rightarrow-i \nabla$, and adopting natural units $(\hbar=c=1)$, we are left with the following differential equation:

$$
\left[\frac{1}{2 \mu}\left(-\frac{d^{2}}{d r^{2}}+\frac{\ell(\ell+1)}{r^{2}}\right)+V^{(0)}(r)\right] y(r)=E y(r)
$$

We point out that in the second version of the model, when we include the spin-spin interaction as a non-perturbative term, the potential actually has spin operators, but as it will be discussed along the text, the spin operator will only contribute with a constant coefficient, just like the orbital momentum operator $\mathbf{L}^{2}$ generates the constant $\ell(\ell+1)$, therefore the potential ends up being a function only of the radial coordinate again.

Regarding the angular part of the wavefunction, we will consider only pure states where $\ell$ (orbital), $S$ (total spin), and $J$ (total angular momentum) are good quantum numbers. Then the wavefunction will be composed of a radial part, and an angular part which comes from the coupling of spherical harmonics and spin functions into a specific value of $J$, (but the values of $\ell$ and $S$ are kept fixed, so only the z-components mix).

From the relativistic point of view, only $J$ is a good quantum number, then in the most general case the total wavefunction could be a mix of different values of $\ell$ and $S$ that lead to the same $J$. The tensor operator for instance, has non-diagonal matrix elements that give rise to a mixing of states with $\ell$ differing by two units (a D-wave state can mix with a $\mathrm{S}$-wave, for example), but for the purpose of our model of tetraquarks (and for mesons or diquarks as well) it is enough to consider pure states, which account for the main contribution in the calculations. This will be further discussed in Section 2.7 and Chapter 3. 
There are still flavor and color parts on the complete wavefunction of the bound state. Since only single flavor states will be considered, the flavor part won't be used in any calculation, except indirectly, in the fact that the quark flavor is related to the quark mass. The color state will be considered separately and discussed in Section 2.4, because it only affects the values of parameters in the potential.

Then, by solving the eigenvalue equation (2.4), one can obtain the interaction energy $E$ and the wavefunction $y(r)$ of the 2-body system under consideration, where both depend on the number of nodes of the wavefunction $n$ (or principal quantum number $N=n+1$ ), on the orbital angular momentum number $\ell$, and in the case of the spin-spin correction included in $V^{(0)}$, they will also depend on the total spin $S$ and the constituents spins $S_{1}$ and $S_{2}$.

Since the Schrödinger equation has no analytical solution with the potentials we are going to use, one option is to solve it numerically. For that, we developed a code written in the software Mathematica based on Ref. [61]. The original notebook was built specially to solve Schrödinger equation for bound states, allowing one to choose as input the number os nodes, the orbital angular momentum, the mass values and precision. We improved it with many additional features that allows one to automatically compute the values of the spindependent corrections, root mean square radius, wavefunction at the origin, normalization factor, total mass, constituents velocities, expectation values of each term in the potential, and to plot the wavefunctions and potentials. We also adapted our program to perform a fitting procedure of the charmonium experimental data in order to determine the best sets of parameters to be used in the potentials (and its extensions to diquarks and tetraquarks), and another adaptation was made to automatically plot the mass spectrum compared to experimental values.

In the end, to calculate the total mass of a particular state we will sum the constituents rest mass to the interaction energy $E$ and the perturbative spin-dependent corrections.

For any charmonium meson $\left(c \bar{c}\right.$, where $\left.m_{c}=m_{\bar{c}}\right)$ we have:

$$
M(c \bar{c})=2 m_{c}+E_{c \bar{c}}+\left\langle V_{S p i n}^{(1)}\right\rangle_{c \bar{c}}
$$

Then for the diquark we will do the analogous calculation adapting the potential for its color structure (and the antidiquark calculation is equal to the one for diquark):

$$
M(c c)=2 m_{c}+E_{c c}+\left\langle V_{S p i n}^{(1)}\right\rangle_{c c}
$$

Lastly, considering the factorization of the 4-body problem into three 2-body problems, the all-charm diquark-antidiquark tetraquark (denoted by $T_{4 c}$ ), will be treated as a 2body system, with the masses of the diquark and antidiquark as input. Then besides the considerations regarding the potential parameters and spin interactions (discussed along the text), the calculation is again analogous to the charmonium:

$$
M\left(T_{4 c}\right)=m_{c c}+m_{\bar{c} \bar{c}}+E_{[c c][\bar{c} \bar{c}]}+\left\langle V_{\text {Spin }}^{(1)}\right\rangle_{[c c][\bar{c} \bar{c}]}
$$

One interesting point about the non-relativistic approach, is that even though the charmonium systems aren't completely non-relativistic, a surprisingly good reproduction of its mass spectrum can be obtained. As analysed in Ref. [62], where a charmonium model is developed with completely relativistic energy and also with non-relativistic kinetic energy, a good agrement with the experimental data can be obtained with both methods, just by using a different set of parameters in the effective potential employed. As we will show in Chapter 4, the second version of the model fits very well the charmonium spectrum, so (within our framework) it probably yields good estimates for the diquark masses, and also 
for the tetraquarks, when the system becomes even less relativistic due to the heavier mass of the diquarks, if compared to the charm quark mass.

\subsection{Constituents Velocities}

Another interesting information that can be used to evaluate the non-relativistic approximation is the velocity of the constituents in each of the systems in consideration, like the quark velocity inside the meson or the diquark, or the diquark velocity inside the tetraquark. As presented in Ref. [63] (where one can also find a numerical package for solving Schrödinger equation in heavy quarkonium context with the Numerov method), the velocity calculation can be performed using the kinetic energy, which can be calculated with two different methods.

First note that since we consider the system in the center of mass frame, the radial coordinate $\mathbf{r}=\mathbf{r}_{\mathbf{1}}-\mathbf{r}_{\mathbf{2}}$ is the distance between the constituents 1 and 2 . We denote the constituent velocity inside the bound state by $|\mathbf{v}| \equiv\left|\mathbf{v}_{\mathbf{1}}\right|=\left|\mathbf{v}_{\mathbf{2}}\right|$ that relates with the velocity relative to the CM by $\mathbf{v}_{\mathbf{r e l}}=2 \mathbf{v}_{\mathbf{1}}=-2 \mathbf{v}_{\mathbf{2}}$, and this velocity relates with the kinetic energy relative to the $\mathrm{CM}$ by:

$$
T=\frac{1}{2} \mu\left\langle\mathbf{v}_{\text {rel }}{ }^{2}\right\rangle=\frac{1}{2} \mu\left\langle(2 \mathbf{v})^{2}\right\rangle \quad \Longrightarrow \quad\left\langle\mathbf{v}^{2}\right\rangle=\frac{1}{2 \mu}\langle T\rangle
$$

Then we can calculate the mean square velocity using the Hamiltonian:

$$
\begin{aligned}
& E=\langle H\rangle=\langle T\rangle+\left\langle V^{(0)}(r)\right\rangle \quad \Longrightarrow \quad\langle T\rangle=E-\left\langle V^{(0)}(r)\right\rangle \\
& \left\langle\mathbf{v}^{2}\right\rangle=\frac{1}{2 \mu}\left(E-\left\langle V^{(0)}(r)\right\rangle\right)
\end{aligned}
$$

The Viral Theorem can be used instead of the Hamiltonian to obtain the kinetic energy:

$$
\begin{aligned}
& \langle T\rangle=\frac{1}{2}\left\langle\mathbf{r} \cdot \nabla V^{(0)}(\mathbf{r})\right\rangle \quad \Longrightarrow \quad\langle T\rangle=\frac{1}{2}\left\langle r \frac{d}{d r} V^{(0)}(r)\right\rangle \quad \text { for central potentials. } \\
& \left\langle\mathbf{v}^{2}\right\rangle=\frac{1}{4 \mu}\left\langle r \frac{d}{d r} V^{(0)}(r)\right\rangle
\end{aligned}
$$

Remember that for equal masses we have $\mu=m / 2$. For the diquark velocity considered as the constituent of the tetraquark (treated as a 2-body system), the calculation is analogous. In order to estimate these velocities we use the energy eigenvalue $\mathrm{E}$, without perturbative corrections. Since we use units where $c=\hbar=1$, these velocities will be already in the form of $v^{2} / c^{2}$, which is ideal to see how non-relativistic is the system.

\subsection{The Hamiltonian}

There are several models of quarkonium in the literature, which employ different theoretical assumptions and mathematical methods. We will adopt a simple model that allow us to obtain a reasonable agreement with charmonium experimental data and analyse many interesting features of the systems we are going to study, while the theoretical background is kept at modest level that can be reduced to mostly non-relativistic quantum mechanics. The deduction of the interactions used in this model can be found in Refs. [59, 60]. These interactions are also presented in Ref. [64] and in Ref. [65] one can find similar and alternative approaches including deductions, from where we got the idea of removing one term from the 
spin-orbit interaction. The modification of the spin-spin interaction of Model 1, which we will denote by Model 2, can be found in Refs. [66, 67] which are partially related with the relativistic model of Ref. [68]. That modification was also employed in Ref. [69].

\subsubsection{Model 1}

In the first approach we will denote the zero-order potential by $V_{1}^{(0)}(r)$, adopting a Coulomb plus linear form, where the Coulomb term is the main contribution of a vector exchange interaction (one gluon exchange), and the linear term is assumed to come from a scalar exchange, responsible for confinement, an effect not yet well understood in fundamental QCD.

$$
\begin{aligned}
V_{1}^{(0)} & =V_{V}^{(0)}+V_{S}^{(0)} \\
V_{1}^{(0)}(r) & =\kappa_{s} \frac{\alpha_{s}}{r}+b r
\end{aligned}
$$

In the above equation the parameter $\kappa_{s}$ (sometimes called "color factor", which can be negative or positive) is related to the color configuration of the system, and it will be calculated in Section 2.4. The parameter $\alpha_{s}$ is the QCD analogue (the index $s$ stands for "strong") to the fine structure constant of the eletromagnetic interactions, and it will be discussed in Section 2.5. The parameter $b$ is sometimes called the "string tension", and it is related to the strength of the confinement. One could also add a constant term, which act as a point-zero energy, like we encounter, for instance, in the quantum harmonic oscillator.

Except for the color factor $\kappa_{s}$, that can be calculated considering a specific color state and the gluon exchange, the $\alpha_{s}$ and $b$ can only be estimated with theoretical assumptions. Their values are somewhat constrained by QCD, lattice simulations and the quarkonium models themselves, where they are usually obtained from fitting the experimental data, using experimentally well-established states as input. The fitting procedure adopted in this work will be discussed in Chapter 4 .

Based on the Breit-Fermi Hamiltonian for one gluon exchange [59, 60, 65] we introduce tree spin-dependent terms, which will be treated with first-order perturbation theory in Model 1, by adding their matrix elements as corrections to the energy.

$$
V_{\text {Spin }}^{(1)}=V_{S S}^{(1)}+V_{L S}^{(1)}+V_{T}^{(1)}, \quad(\text { for model } 1)
$$

There are three spin-dependent terms: $V_{S S}^{(1)}$ (Spin-Spin), $V_{L S}^{(1)}$ (Spin-Orbit) and $V_{T}^{(1)}$ (Tensor), which will be discussed in detail in Chapter 3. For equal masses $m_{1}=m_{2}=m$, they are given by:

$$
\begin{aligned}
V_{S S}^{(1)} & =C_{S S}(r) \mathbf{S}_{\mathbf{1}} \cdot \mathbf{S}_{\mathbf{2}} \\
V_{L S}^{(1)} & =C_{L S}(r) \mathbf{L} \cdot \mathbf{S} \\
V_{T}^{(1)} & =C_{T}(r)\left(\frac{\left(\mathbf{S}_{\mathbf{1}} \cdot \mathbf{r}\right)\left(\mathbf{S}_{\mathbf{2}} \cdot \mathbf{r}\right)}{\mathbf{r}^{2}}-\frac{1}{3}\left(\mathbf{S}_{\mathbf{1}} \cdot \mathbf{S}_{\mathbf{2}}\right)\right)
\end{aligned}
$$

where the coefficients depend on the vector $V_{V}$ and scalar $V_{S}$ contributions of the potential 
on eq. (2.13), (the derivatives are explicitly calculated in Chapter 3):

$$
\begin{aligned}
C_{S S}(r) & =\frac{2}{3 m^{2}} \nabla^{2} V_{V}(r)=-\frac{8 \kappa_{s} \alpha_{s} \pi}{3 m^{2}} \delta^{3}(r) \\
C_{L S}(r) & =\frac{1}{2 m^{2}} \frac{1}{r}\left[3 \frac{d V_{V}(r)}{d r}-\frac{d V_{S}(r)}{d r}\right]=-\frac{3 \kappa_{s} \alpha_{s}}{2 m^{2}} \frac{1}{r^{3}}-\frac{b}{2 m^{2}} \frac{1}{r} \\
C_{T}(r) & =\frac{1}{m^{2}}\left[\frac{1}{r} \frac{d V_{V}(r)}{d r}-\frac{d^{2} V_{V}(r)}{d r^{2}}\right]=-\frac{12 \kappa_{s} \alpha_{s}}{4 m^{2}} \frac{1}{r^{3}}
\end{aligned}
$$

where $m$ is the constituent mass of the 2-body problem (charm quark, or diquark). The second term incorporated in the spin-orbit correction (proportional to the scalar contribution) is a Thomas precession which follows from the assumption that the confining interaction comes from a Lorentz scalar [59, 60, 66, 67].

These spin-dependent terms are proportional to $1 / \mathrm{m}^{2}$, what justifies their treatment as first-order perturbation corrections in heavy quark bound states, and the fact that they depend on the spin and orbital angular momentum makes them interesting to study the splitting structure of the spectrum and its dependence on the quantum numbers under consideration.

For simplicity, we won't consider momentum-dependent corrections, like the lowest-order relativistic correction in the kinetic energy proportional to $\mathbf{p}^{4}$, since reasonable agreement with experimental data can already be obtained with a proper choice of parameters. For the same reason spin-independent corrections (as the one presented in Ref. [60]) won't be considered either.

\subsubsection{Model 2}

In Chapter 4 we will present and discuss the application of this model to study the charmonium states (mesons $c \bar{c}$ ). We will see that a better agreement between predicted states and experimental data can be obtained by including the spin-spin interaction in the zeroth-order potential used in the Schrödinger equation (as done in Refs. [66, 67]), with the artifact of replacing the Dirac delta by a gaussian function which introduces a new parameter $\sigma$. The spin-spin term becomes:

$$
V_{S S}^{(0)}=-\frac{8 \pi \kappa_{s} \alpha_{s}}{3 m^{2}}\left(\frac{\sigma}{\sqrt{\pi}}\right)^{3} \mathrm{e}^{-\sigma^{2} r^{2}} \mathbf{S}_{\mathbf{1}} \cdot \mathbf{S}_{\mathbf{2}}
$$

Then the zeroth-order potential will be $V_{2}^{(0)}$ :

$$
\begin{aligned}
V_{2}^{(0)} & =V_{V}^{(0)}+V_{S}^{(0)}+V_{S S}^{(0)} \\
V_{2}^{(0)}(r) & =\kappa_{s} \frac{\alpha_{s}}{r}+b r-\frac{8 \pi \kappa_{s} \alpha_{s}}{3 m^{2}}\left(\frac{\sigma}{\sqrt{\pi}}\right)^{3} \mathrm{e}^{-\sigma^{2} r^{2}}\left(\mathbf{S}_{\mathbf{1}} \cdot \mathbf{S}_{\mathbf{2}}\right)
\end{aligned}
$$

When the term $\mathbf{S}_{\mathbf{1}} \cdot \mathbf{S}_{\mathbf{2}}$ acts on the wavefunction it will generate a constant factor (as discussed in Chapter 3), so we still have a potential as function of only the $r$ coordinate.

The spin-orbit and tensor corrections are still treated with perturbation theory, then:

$$
V_{\text {Spin }}^{(1)}=V_{L S}^{(1)}+V_{T}^{(1)}, \quad(\text { for model 2) }
$$




\subsection{Color Factor $\kappa_{s}$}

When describing bound states of quarks, we need to analyse the color part of the wavefunction that describes the color state of the constituents (quarks and/or antiquarks), in a similar way we describe the total spin state of fermions. In our model the color state is used to calculate the color factor that appears in the static potential.

The deduction of the "Coulomb" term of the Cornell potential can be obtained comparing the one gluon exchange in (perturbative) Quantum Chromodynamics (QCD) to the one photon exchange in Quantum Electrodynamics (QED), as discussed in Section 8.4 of the textbook [1] and presented in the text below. More compact calculations can be found in Refs. [59, 60, 65].

\subsubsection{Quark - Antiquark}

First let us analyse the amplitude of the interaction of a quark and an antiquark of different flavor that exchange one gluon. The first-order Feynman diagram of this process is shown in Figure 2.1:

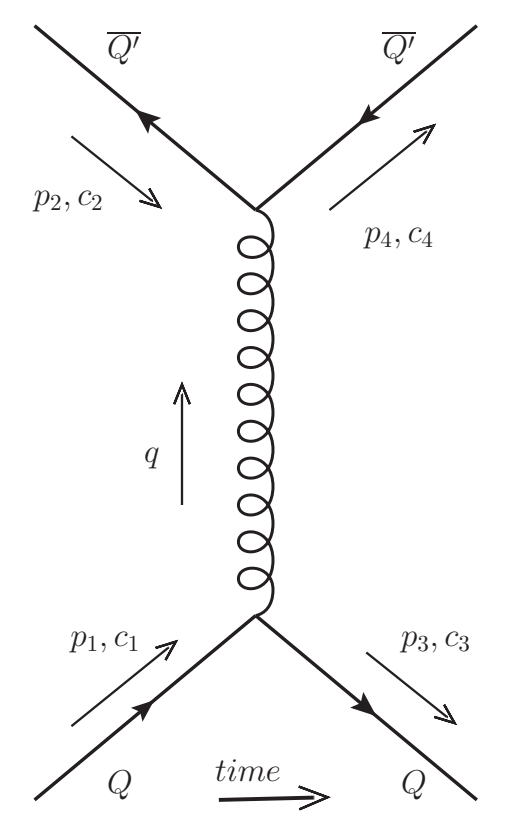

Figure 2.1: One gluon exchange diagram for $Q \overline{Q^{\prime}} \rightarrow Q \overline{Q^{\prime}}$.

Using the Feynman Rules of QCD [1] one can build the interaction amplitude:

$$
\mathfrak{M}_{q \bar{q}}=i\left[\bar{u}(3) c_{3}^{\dagger}\right]\left[-i g_{s} \frac{\lambda^{\alpha}}{2} \gamma^{\mu}\right]\left[u(1) c_{1}\right]\left[\frac{-i g_{\mu \nu} \delta^{\alpha \beta}}{q^{2}}\right]\left[\bar{v}(2) c_{2}^{\dagger}\right]\left[-i g_{s} \frac{\lambda^{\beta}}{2} \gamma^{\nu}\right]\left[v(4) c_{4}\right]
$$

where $q=p_{1}-p_{3}=p_{4}-p_{2}$.

Working it out we get the same amplitude of the QED process of electron-positron scattering, only changing $g_{e} \rightarrow g_{s}$ and with an additional "color factor" $f_{s}$ :

$$
\mathfrak{M}_{q \bar{q}}=-\frac{g_{s}^{2}}{q^{2}}\left[\bar{u}(3) \gamma^{\mu} u(1)\right]\left[\bar{v}(2) \gamma_{\mu} v(4)\right]\left[\frac{1}{4}\left(c_{3}^{\dagger} \lambda^{\alpha} c_{1}\right)\left(c_{2}^{\dagger} \lambda^{\alpha} c_{4}\right)\right]
$$




$$
f_{s}^{q-\bar{q}}=\frac{1}{4} \sum_{\alpha}\left(c_{3}^{\dagger} \lambda^{\alpha} c_{1}\right)\left(c_{2}^{\dagger} \lambda^{\alpha} c_{4}\right)
$$

The repeated $\alpha$ indices in the notation of eq. (2.25) imply a summation over the 8 gluon colors: $\alpha=1,2, \ldots, 8$, which is shown explicitly in eq. (2.26).

The color of each object can be written with the following fundamental representation:

$$
c=\left(\begin{array}{l}
1 \\
0 \\
0
\end{array}\right) \text { for red, }\left(\begin{array}{l}
0 \\
1 \\
0
\end{array}\right) \text { for blue, }\left(\begin{array}{l}
0 \\
0 \\
1
\end{array}\right) \text { for green. }
$$

The scattering of two unity electric charges $e$ in QED (due to one photon exchange), can be associated with the electromagnetic Coulomb potential:

$$
V_{\text {Coulomb }}= \begin{cases}-\frac{e^{2}}{4 \pi \epsilon_{0}} \frac{1}{r} & =-\alpha_{e} \hbar c \frac{1}{r} \quad \text { for opposite sign charges, } \\ +\frac{e^{2}}{4 \pi \epsilon_{0}} \frac{1}{r} & =+\alpha_{e} \hbar c \frac{1}{r} \quad \text { for same sign charges. }\end{cases}
$$

Similarly, the one gluon exchange between quark and antiquark (carrying color charge and anticolor charge, respectively) can be associated with a "Coulomb" potential, where the following connection is made (notice the minus sign stands for charge-anticharge interaction):

$$
-\frac{e^{2}}{\hbar c 4 \pi \epsilon_{0}}=-\alpha_{e}(\mathrm{QED}) \longleftrightarrow-f_{s}^{q-\bar{q}} \alpha_{s}(\mathrm{QCD})
$$

Then in order to clarify the discussion about the "color factor", we will define the parameter $\kappa_{s}$, in the "Coulomb" part of the Cornell potential in eq. (2.13), already with the minus sign included when the interaction is between charge and anticharge:

$$
\kappa_{s}=\left\{\begin{array}{ccc}
-f_{s}^{q-\bar{q}}, & \text { for } & q-\bar{q} \\
+f_{s}^{q-q}, & \text { for } & q-q \\
+f_{s}^{\bar{q}-\bar{q}} & \text { for } & \bar{q}-\bar{q}
\end{array}\right.
$$

So the Coulomb term, or vector contribution $V_{V}(r)=\kappa_{s} \alpha_{s} \frac{1}{r}$, in the Cornell potential (2.13) will be:

$$
\begin{aligned}
V_{V}^{q-\bar{q}}(r) & =-f_{s}^{q-\bar{q}} \alpha_{s} \frac{1}{r} \\
V_{V}^{q-q}(r) & =+f_{s}^{q-q} \alpha_{s} \frac{1}{r} \\
V_{V}^{\bar{q}-\bar{q}}(r) & =+f_{s}^{\bar{q}-\bar{q}} \alpha_{s} \frac{1}{r}
\end{aligned}
$$

where we already made $\hbar=c=1$. From now on, when we say "color factor", we mean $f_{s}$, apart from the + or - sign that must be included to get the $\kappa_{s}$ parameter. As we will see, 
the color factor $f_{s}$ can result to be negative.

This sign rule can be thought as an analogy to the Coulomb potential in classical electromagnetism. The interaction between quark-quark or antiquark-antiquark carries a + sign because it is an interaction between two color charges or two anticolor charges (respectively), analogous to the case of same sign charges in electromagnetic process (i.e. both positive or both negative electric charges, as one encounters, for example, in the electronmuon scattering: both negative). For quark-antiquark, the color-anticolor interaction implies an extra minus sign in the potential, analogous to the case of opposite electric charges (like in electron-positron scattering: negative and positive, respectively).

But since we are dealing with $S U(3)$ color symmetry, there will be more possibilities than the only two in the $U(1)$ symmetry of QED. The possible color states of a quark-antiquark system are obtained by the combination of 3 colors and 3 anticolors, while for quark-quark (antiquark-antiquark) we have 3 colors (anticolors) combined with 3 colors (anticolors).

The "anti" of antitriplet (in the following paragraph) means that the colors are combined into an antisymmetric triplet color state (see eq. (2.50) in the next section), in the same sense that two spin $1 / 2$ particles can combine into a spin 0 antisymmetric state. The "overline" bar in eq. (2.32) refers to the charge conjugated representation [70].

In group theory language the direct product of a color triplet (the three color charges of QCD) and an anticolor triplet results in the direct sum of a singlet and an octet, while the direct product of a color triplet and another color triplet results in the direct sum of an antitriplet and a sextet, and in the same way, the direct product of an anticolor triplet and another anticolor triplet results in the direct sum of an anticolor antitriplet and an anticolor sextet, which are the conjugates from the combinations obtained with color, therefore they can be combined to form a color singlet (see Refs. [70, 71, 72] for instance):

$$
\begin{aligned}
& |q \bar{q}\rangle: 3 \otimes \overline{3}=1 \oplus 8 \\
& |q q\rangle: 3 \otimes 3=\overline{3} \oplus 6 \\
& |\overline{q q}\rangle: \overline{3} \otimes \overline{3}=3 \oplus \overline{6}
\end{aligned}
$$

Remember that quark and antiquark can be combined into a color singlet state, which is an observable: the mesons $q \bar{q}$. Also three quarks (or three antiquarks) can be combined into a color singlet state: the baryons $q q q$, like protons and neutrons. With that in mind, the tetraquark color state is built in a similar manner as in a meson: first combining two quarks into a color antitriplet diquark and two antiquarks into an anticolor antitriplet antidiquark, then the diquark and antidiquark are combined into a singlet (therefore, observable) tetraquark! The same procedure could be done with the sextet states, but as we shall see after calculating the color factors, the antitriplet state is attractive, while the sextet is repulsive. The singlet color factor is also attractive. Even if one consider a four-to-four body interaction with all color combinations that appear, the triplet contribution tends to be dominant. Also, if still in the diquark factorization one consider a mixing of antitriplet and sextet, the sextet contribution usually appears mixed in a few states, while the antitriplet can alone build pure states [50]. In some cases the sextet contribution was investigated and found to be negligible (see Refs. [73, 74]).

Equation (2.32) tells us that a quark-antiquark system can be in an octet or a singlet color state (but only the singlet is an observable, due to color confinement). We will start with a simplified calculation of the octet color factor. The possible color combinations of the 
color octet are listed in eq. (2.33). These are also the color states of the 8 types of gluons:

$$
\begin{aligned}
|1\rangle_{8} & =(r \bar{b}+b \bar{r}) / \sqrt{2} & |5\rangle_{8} & =-i(r \bar{g}-g \bar{r}) / \sqrt{2} \\
|2\rangle_{8} & =-i(r \bar{b}-b \bar{r}) / \sqrt{2} & |6\rangle_{8} & =(b \bar{g}+g \bar{b}) / \sqrt{2} \\
|3\rangle_{8} & =(r \bar{r}-b \bar{b}) / \sqrt{2} & |7\rangle_{8} & =-i(b \bar{g}-g \bar{b}) / \sqrt{2} \\
|4\rangle_{8} & =(r \bar{g}+g \bar{r}) / \sqrt{2} & |8\rangle_{8} & =(r \bar{r}+b \bar{b}-2 g \bar{g}) / \sqrt{6}
\end{aligned}
$$

The color factor will depend only on the "color multiplet", for example, all the eight octet states of eq. (2.33) will result in the same color factor, characteristic of the color octet. The same is true for the antitriplet and sextet that we will see for the quark-quark interaction in Section 2.4.2. The simplified octet state $r \bar{b}$ leads to the octet color factor as well, and it is a good way to start the color factor calculations because it will be easy to handle. The quark will carry the color $r$ and the antiquark will carry the anticolor $\bar{b}$. In this case, color can not change in the vertex of the interaction because it must be conserved, and neither quark can carry the anticolor nor antiquark can carry the color. Then the final color state of each one will be the same after the interaction:

$$
c_{1}=c_{3}=r=\left(\begin{array}{l}
1 \\
0 \\
0
\end{array}\right), \quad c_{2}=c_{4}=\bar{b}=\left(\begin{array}{l}
0 \\
1 \\
0
\end{array}\right)
$$

Notice that there is no distinction between color and anticolor in this fundamental representation. But their nature will be considered in the color factor due to the order they appear when we build the amplitude based on the Feynman diagrams (compare the color factor for $q \bar{q}$ in eq. (2.26) with its analogue for $q q$ in eq. (2.49)).

In order to calculate the color factors we will need the Gell-Mann matrices, that are to $S U(3)$ what the Pauli matrices are to $S U(2) i$. e., they are the generators (when multiplied by a factor $1 / 2$ ) of the elements of that group of symmetry:

$$
\begin{gathered}
\lambda^{1}=\left(\begin{array}{lll}
0 & 1 & 0 \\
1 & 0 & 0 \\
0 & 0 & 0
\end{array}\right) \\
\lambda^{2}=\left(\begin{array}{ccc}
0 & -i & 0 \\
i & 0 & 0 \\
0 & 0 & 0
\end{array}\right) \quad \lambda^{3}=\left(\begin{array}{ccc}
1 & 0 & 0 \\
0 & -1 & 0 \\
0 & 0 & 0
\end{array}\right) \\
\lambda^{4}=\left(\begin{array}{lll}
0 & 0 & 1 \\
0 & 0 & 0 \\
1 & 0 & 0
\end{array}\right) \quad \lambda^{5}=\left(\begin{array}{ccc}
0 & 0 & -i \\
0 & 0 & 0 \\
i & 0 & 0
\end{array}\right) \quad \lambda^{6}=\left(\begin{array}{ccc}
0 & 0 & 0 \\
0 & 0 & 1 \\
0 & 1 & 0
\end{array}\right) \\
\lambda^{7}=\left(\begin{array}{ccc}
0 & 0 & 0 \\
0 & 0 & -i \\
0 & i & 0
\end{array}\right) \quad \lambda^{8}=\frac{1}{\sqrt{3}}\left(\begin{array}{ccc}
1 & 0 & 0 \\
0 & 1 & 0 \\
0 & 0 & -2
\end{array}\right)
\end{gathered}
$$

Using eq. (2.34) in eq. (2.26) and the Gell-Mann matrices (eq. 2.35), we get:

$$
\begin{aligned}
f_{8}^{q-\bar{q}} & \left.=\frac{1}{4}\left[\left(\begin{array}{lll}
1 & 0 & 0
\end{array}\right) \lambda^{\alpha}\left(\begin{array}{l}
1 \\
0 \\
0
\end{array}\right)\right]\left[\begin{array}{lll}
0 & 1 & 0
\end{array}\right) \lambda^{\alpha}\left(\begin{array}{l}
0 \\
1 \\
0
\end{array}\right)\right]=\frac{1}{4} \sum_{\alpha}\left(\lambda_{11}^{\alpha} \lambda_{22}^{\alpha}\right) \\
& =\frac{1}{4}\left(\lambda_{11}^{3} \lambda_{22}^{3}+\lambda_{11}^{8} \lambda_{22}^{8}\right)=\frac{1}{4}[(1)(-1)+(1 / \sqrt{3})(1 / \sqrt{3})]=-\frac{1}{6}
\end{aligned}
$$

So this is the color factor for the octet color state in $q \bar{q}$ interaction. Notice that we changed the subscript from "s" (that stands for "strong interaction") to give place to identify 
the multiplet ("8" for octet, "1" for singlet, ahead). Remember that there is an additional minus sign because we have "opposite" charges (color and anticolor). Then the $\kappa_{s}$ parameter in the potential will be:

$$
\kappa_{s}(\text { octet })=-f_{8}^{q-\bar{q}}=-\left(-\frac{1}{6}\right)=+\frac{1}{6}
$$

Now the color singlet state is:

$$
|1\rangle_{1}=(r \bar{r}+b \bar{b}+g \bar{g}) / \sqrt{3}
$$

Notice that besides being "colorless", by which we mean it has the "same amount" of each color and respective anticolor, it is also symmetric by the permutation of any two pairs of color-anticolor. The octet states $|3\rangle_{8}$ and $|8\rangle_{8}$ in eq. (2.33) are "colorless", but not color singlets.

The singlet color factor calculation is a bit trickier. Since the incoming quark-antiquark pair is in the color singlet state, the color factor starts as a sum of three terms:

$$
\begin{aligned}
f_{1}^{q-\bar{q}}= & \frac{1}{4} \frac{1}{\sqrt{3}}\left\{\left[c_{3}^{\dagger} \lambda^{\alpha}\left(\begin{array}{l}
1 \\
0 \\
0
\end{array}\right)\right]\left[\left(\begin{array}{lll}
(1 & 0 & 0
\end{array}\right) \lambda^{\alpha} c_{4}\right]+\left[c_{3}^{\dagger} \lambda^{\alpha}\left(\begin{array}{l}
0 \\
1 \\
0
\end{array}\right)\right]\left[\begin{array}{lll}
0 & 1 & 0
\end{array}\right) \lambda^{\alpha} c_{4}\right] \\
& \left.+\left[c_{3}^{\dagger} \lambda^{\alpha}\left(\begin{array}{l}
0 \\
0 \\
1
\end{array}\right)\right]\left[\left(\begin{array}{lll}
0 & 0 & 1
\end{array}\right) \lambda^{\alpha} c_{4}\right]\right\}
\end{aligned}
$$

In equation (2.39) we have put the initial color states $c_{1}$ and $c_{2}$. Now we have to consider that each one of the three terms can have any of the three "colorless" coloranticolor possibilities, but the outgoing quark-antiquark pair must still be in the singlet state. Therefore, we get 9 terms (each one carrying a summation in $\alpha$ ), which can be written compactly as:

$$
f_{1}^{q-\bar{q}}=\frac{1}{4} \frac{1}{\sqrt{3}} \frac{1}{\sqrt{3}} \sum_{\alpha}\left(\lambda_{i j}^{\alpha} \lambda_{j i}^{\alpha}\right)=\frac{1}{12} \operatorname{Tr}\left(\lambda^{\alpha} \lambda^{\alpha}\right)
$$

And using the following relation:

$$
\operatorname{Tr}\left(\lambda^{\alpha} \lambda^{\beta}\right)=2 \delta^{\alpha \beta} \Rightarrow \operatorname{Tr}\left(\lambda^{\alpha} \lambda^{\alpha}\right)=16
$$

We finally get the singlet color factor in $q \bar{q}$ interaction:

$$
f_{1}^{q-\bar{q}}=\frac{4}{3}
$$

Again, remember that there is an additional minus sign because we have "opposite" charges (color and anticolor). Then the $\kappa_{s}$ parameter in the potential will be:

$$
\kappa_{s}(\text { singlet })=-f_{1}^{q-\bar{q}}=-\left(\frac{4}{3}\right)=-\frac{4}{3}
$$

One could argue that for quarks of the same flavor there is another first-order diagram that could contribute to the amplitude, in which the $Q \bar{Q}$ virtually annihilates into a gluon: 


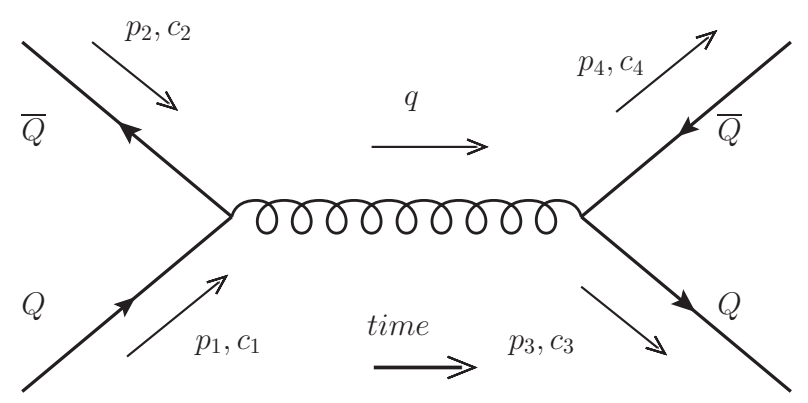

Figure 2.2: Virtual annihilation $Q \bar{Q} \rightarrow$ gluon $\rightarrow Q \bar{Q}$.

The amplitude for this diagram reads:

$$
\mathfrak{M}_{q \bar{q} \rightarrow g \rightarrow q \bar{q}}=i\left[\bar{v}(2) c_{2}^{\dagger}\right]\left[-i g_{s} \frac{\lambda^{\alpha}}{2} \gamma^{\mu}\right]\left[u(1) c_{1}\right]\left[\frac{-i g_{\mu \nu} \delta^{\alpha \beta}}{q^{2}}\right]\left[\bar{u}(3) c_{3}^{\dagger}\right]\left[-i g_{s} \frac{\lambda^{\beta}}{2} \gamma^{\nu}\right]\left[v(4) c_{4}\right]
$$

where $q=p_{1}+p_{2}=p_{3}+p_{4}$.

Once more we can extract the color factor by comparison with the analogue process in QED: the eletron-positron virtual annihilation. Working it out we get:

$$
\begin{gathered}
\mathfrak{M}_{q \bar{q} \rightarrow g \rightarrow q \bar{q}}=-\frac{g_{s}^{2}}{q^{2}}\left[\bar{v}(2) \gamma^{\mu} u(1)\right]\left[\bar{u}(3) \gamma_{\mu} v(4)\right]\left[\frac{1}{4}\left(c_{2}^{\dagger} \lambda^{\alpha} c_{1}\right)\left(c_{3}^{\dagger} \lambda^{\alpha} c_{4}\right)\right] \\
f_{s}^{q \bar{q} \rightarrow g \rightarrow q \bar{q}}=\frac{1}{4} \sum_{\alpha}\left(c_{2}^{\dagger} \lambda^{\alpha} c_{1}\right)\left(c_{3}^{\dagger} \lambda^{\alpha} c_{4}\right)
\end{gathered}
$$

Notice this color factor is different from that of one gluon exchange for $q \bar{q}$ in eq. (2.26), and from that of one gluon exchange for $q q$ in eq. (2.49) which we will see in Section 2.4.2.

For $q \bar{q}$ we saw that the color state can be an octet or a singlet, but since only the singlet can be a observable (meson), we will calculate the color factor of virtual annihilation only for the singlet color state. Using eq. (2.38) in eq. (2.46) we get:

$$
\begin{aligned}
f_{s}^{q \bar{q} \rightarrow g \rightarrow q \bar{q}} & =\frac{1}{4} \frac{1}{\sqrt{3}} \frac{1}{\sqrt{3}}\left[\left(\begin{array}{lll}
1 & 0 & 0
\end{array}\right) \lambda^{\alpha}\left(\begin{array}{l}
1 \\
0 \\
0
\end{array}\right)+\left(\begin{array}{lll}
0 & 1 & 0
\end{array}\right) \lambda^{\alpha}\left(\begin{array}{l}
0 \\
1 \\
0
\end{array}\right)+\left(\begin{array}{lll}
0 & 0 & 1
\end{array}\right) \lambda^{\alpha}\left(\begin{array}{l}
0 \\
0 \\
1
\end{array}\right)\right] \\
& \times\left[\left(\begin{array}{lll}
1 & 0 & 0
\end{array}\right) \lambda^{\alpha}\left(\begin{array}{l}
1 \\
0 \\
0
\end{array}\right)+\left(\begin{array}{lll}
0 & 1 & 0
\end{array}\right) \lambda^{\alpha}\left(\begin{array}{l}
0 \\
1 \\
0
\end{array}\right)+\left(\begin{array}{lll}
0 & 0 & 1
\end{array}\right) \lambda^{\alpha}\left(\begin{array}{l}
0 \\
0 \\
1
\end{array}\right)\right] \\
& =\frac{1}{12} \operatorname{Tr}\left(\lambda^{\alpha}\right) \operatorname{Tr}\left(\lambda^{\alpha}\right)=0
\end{aligned}
$$

Therefore we have shown that this virtual annihilation diagram doesn't contribute to the amplitude of the color singlet (meson) interaction, because its corresponding color factor is zero, which tells us that a singlet (meson) cannot couple to an octet (gluon). 


\subsubsection{Quark - Quark}

The amplitude of the one gluon exchange between two quarks of different flavor can be compared to the one photon exchange between an electron and a muon (which have the same charge sign), and the calculation of the color factor follows the same procedure we saw in Section 2.4.1 for quark-antiquark:

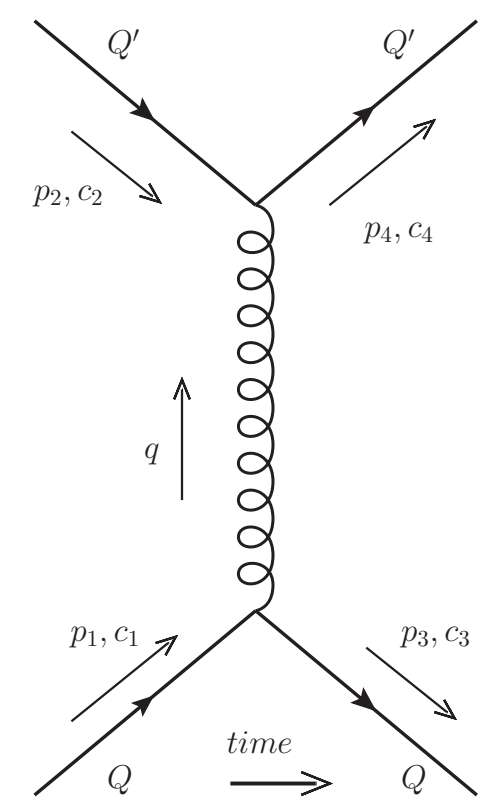

Figure 2.3: One gluon exchange diagram for $Q Q^{\prime} \rightarrow Q Q^{\prime}$.

$$
\begin{gathered}
\mathfrak{M}_{q q}=-\frac{g_{s}^{2}}{q^{2}}\left[\bar{u}(3) \gamma^{\mu} u(1)\right]\left[\bar{u}(4) \gamma_{\mu} u(2)\right]\left[\frac{1}{4} \sum_{\alpha}\left(c_{3}^{\dagger} \lambda^{\alpha} c_{1}\right)\left(c_{4}^{\dagger} \lambda^{\alpha} c_{2}\right)\right] \\
f_{s}^{q-q}=\frac{1}{4} \sum_{\alpha}\left(c_{3}^{\dagger} \lambda^{\alpha} c_{1}\right)\left(c_{4}^{\dagger} \lambda^{\alpha} c_{2}\right)
\end{gathered}
$$

Notice that now we have two particle spinors $u$ instead of a particle spinor $u$ and an antiparticle spinor $v$ (that was the case of $q \bar{q}$ ), and therefore the colors $c_{2}$ and $c_{4}$ are in exchanged positions.

The possible color combinations of the color antitriplet are:

$$
\begin{aligned}
|1\rangle_{\overline{3}} & =(r b-b r) / \sqrt{2} \\
|2\rangle_{\overline{3}} & =(b g-g b) / \sqrt{2} \\
|3\rangle_{\overline{3}} & =(g r-r g) / \sqrt{2}
\end{aligned}
$$

In order to calculate the antitriplet color factor, let us use the color state $|1\rangle_{\overline{3}}=(r b-$ $b r) / \sqrt{2}$ (using any of the others antitriplet color states in eq. (2.50) will result in the same antitriplet color factor). In this color configuration we will have $(r b-b r) \rightarrow(r b-b r)$ which will lead to four terms in the form $\left(c_{1}, c_{2}\right) \rightarrow\left(c_{3}, c_{4}\right)$, corresponding to $r b \rightarrow r b, r b \rightarrow-b r$, $-r b \rightarrow r b$ and $-b r \rightarrow-b r$ (the minus sign will cancel in this last one): 


$$
\begin{aligned}
& f_{\overline{3}}^{q-q}=\frac{1}{4} \frac{1}{\sqrt{2}} \frac{1}{\sqrt{2}}\left\{\left[\left(\begin{array}{lll}
1 & 0 & 0
\end{array}\right) \lambda^{\alpha}\left(\begin{array}{l}
1 \\
0 \\
0
\end{array}\right)\right]\left[\begin{array}{lll}
0 & 1 & 0
\end{array}\right) \lambda^{\alpha}\left(\begin{array}{l}
0 \\
1 \\
0
\end{array}\right)\right] \\
& \left.-\left[\left(\begin{array}{lll}
0 & 1 & 0
\end{array}\right) \lambda^{\alpha}\left(\begin{array}{l}
1 \\
0 \\
0
\end{array}\right)\right]\left[\begin{array}{lll}
1 & 0 & 0
\end{array}\right) \lambda^{\alpha}\left(\begin{array}{l}
0 \\
1 \\
0
\end{array}\right)\right] \\
& \left.-\left[\left(\begin{array}{lll}
1 & 0 & 0
\end{array}\right) \lambda^{\alpha}\left(\begin{array}{l}
0 \\
1 \\
0
\end{array}\right)\right]\left[\begin{array}{lll}
0 & 1 & 0
\end{array}\right) \lambda^{\alpha}\left(\begin{array}{l}
1 \\
0 \\
0
\end{array}\right)\right] \\
& \left.\left.+\left[\left(\begin{array}{lll}
0 & 1 & 0
\end{array}\right) \lambda^{\alpha}\left(\begin{array}{l}
0 \\
1 \\
0
\end{array}\right)\right]\left[\begin{array}{lll}
1 & 0 & 0
\end{array}\right) \lambda^{\alpha}\left(\begin{array}{l}
1 \\
0 \\
0
\end{array}\right)\right]\right\} \\
& =\frac{1}{8} \sum_{\alpha}\left(\lambda_{11}^{\alpha} \lambda_{22}^{\alpha}-\lambda_{21}^{\alpha} \lambda_{12}^{\alpha}-\lambda_{12}^{\alpha} \lambda_{21}^{\alpha}+\lambda_{22}^{\alpha} \lambda_{11}^{\alpha}\right)
\end{aligned}
$$

Notice that we can commute the two last terms to obtain the first two again, then:

$$
\begin{aligned}
f_{\frac{3}{3}}^{q-q} & =\frac{1}{4} \sum_{\alpha}\left(\lambda_{11}^{\alpha} \lambda_{22}^{\alpha}-\lambda_{12}^{\alpha} \lambda_{21}^{\alpha}\right) \\
& =\frac{1}{4}\left(\lambda_{11}^{3} \lambda_{22}^{3}+\lambda_{11}^{8} \lambda_{22}^{8}-\lambda_{12}^{1} \lambda_{21}^{1}-\lambda_{12}^{2} \lambda_{21}^{2}\right) \\
& =\frac{1}{4}[(1)(-1)+(1 / \sqrt{3})(1 / \sqrt{3})-(1)(1)-(-i)(i)]=-\frac{2}{3}
\end{aligned}
$$

This time, we have a "+" sign when changing to $\kappa_{s}$ because of "equal" sign charges (color and color). Therefore the $\kappa_{s}$ parameter in the potential will be equal to the color factor $f_{s}$ :

$$
\kappa_{s}(\text { antitriplet })=+f_{\frac{q}{3}}^{q-q}=+\left(-\frac{2}{3}\right)=-\frac{2}{3}
$$

Finally, for the color sextet we have the following color states:

$$
\begin{aligned}
|1\rangle_{6} & =r r \quad|2\rangle_{6}=b b \quad|3\rangle_{6}=g g \\
|4\rangle_{6} & =(r b+b r) / \sqrt{2} \\
|5\rangle_{6} & =(b g+g b) / \sqrt{2} \\
|6\rangle_{6} & =(g r+r g) / \sqrt{2}
\end{aligned}
$$

Then to calculate the sextet color factor, we will use the color state $|1\rangle_{6}=\operatorname{rr}$ (remember that using any of the others color sextet states in eq. (2.54) will result in the same sextet color factor). So using this state in eq. (2.49) we get:

$$
\begin{aligned}
f_{6}^{q-q} & \left.=\frac{1}{4}\left[\left(\begin{array}{ccc}
1 & 0 & 0
\end{array}\right) \lambda^{\alpha}\left(\begin{array}{l}
1 \\
0 \\
0
\end{array}\right)\right]\left[\begin{array}{lll}
1 & 0 & 0
\end{array}\right) \lambda^{\alpha}\left(\begin{array}{l}
1 \\
0 \\
0
\end{array}\right)\right]=\frac{1}{4} \sum_{\alpha}\left(\lambda_{11}^{\alpha} \lambda_{11}^{\alpha}\right) \\
& =\left(\lambda_{11}^{3} \lambda_{11}^{3}+\lambda_{11}^{8} \lambda_{11}^{8}\right)=[(1)(1)+(1 / \sqrt{3})(1 / \sqrt{3})]=+\frac{1}{3}
\end{aligned}
$$


Once more, due to the + sign for "equal charges", the $\kappa_{s}$ parameter in the potential will be equal to the color factor $f_{s}$ :

$$
\kappa_{s}(\text { sextet })=+f_{6}^{q-q}=+\left(+\frac{1}{3}\right)=+\frac{1}{3}
$$

One could argue that for identical quarks (same flavor) there is another first-order diagram, with "crossed" momentum:

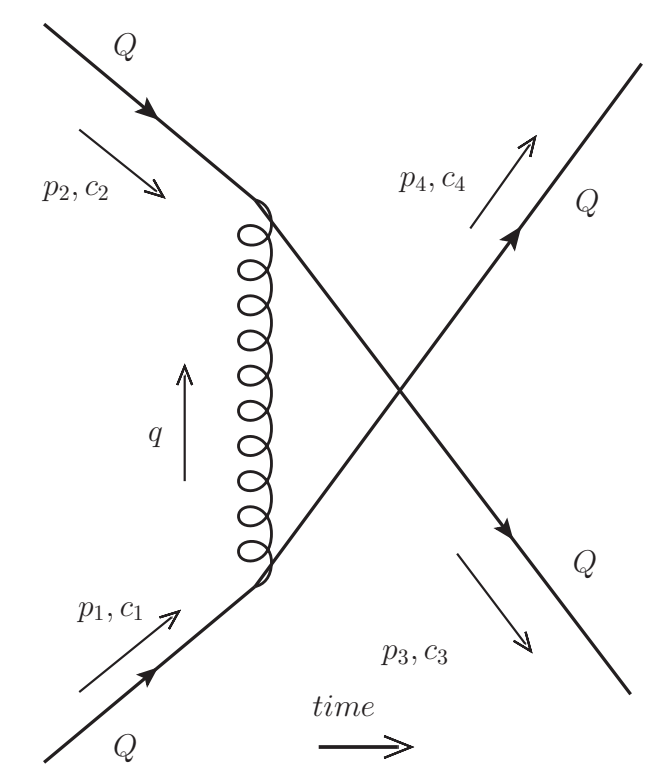

Figure 2.4: OGE diagram with "crossed momentum" for $Q Q \rightarrow Q Q$.

This one is not zero, but if we include it in the amplitude calculation, together with the corresponding statistical factor (dividing by all the possible initial states and summing over all the possible final states), we get exactly the same result as if we had only considered the "regular" diagram, so the previous calculations for the color factor are valid for identical quarks as well.

Lastly, we point out that everything done for quark-quark is similar for antiquarkantiquark interactions, just replacing colors by anticolors. Then the resulting structures of antiquark-antiquark are complementary to the quark-quark ones, as it can be seen in eq. (2.32). So if considered as bound structures (quark-quark as diquark and antiquark-antiquark as antidiquark) they can be combined to form a color singlet as well. Therefore, the diquarkantidiquark interaction (treated as a 2-body problem) is similar to the quark-antiquark, and the color factor to form the color singlet is the same as in a regular meson.

In conclusion, there are two most interesting cases between the four we saw: the singlet color factor (which is used in regular meson potentials, and will be used in our diquarkantidiquark interaction), and the antitriplet color factor, which is the attractive (and dominant) color interaction between quark-quark (and antiquark-antiquark) which we will use to build the diquarks (and antidiquarks), constituents of the final color singlet tetraquark bound state.

Summarizing: for quark-quark (or antiquark-antiquark) we have a positive sign in the Coulomb term of the Cornell potential, but the factor $f_{s}$ (for color antitriplet configuration) 
is negative:

$$
f_{\frac{q}{3}-q}^{q-q} f_{3}^{\bar{q}-\bar{q}}=-\frac{2}{3} \Rightarrow V_{V}(r)=+\left(-\frac{2}{3}\right) \frac{\alpha_{s}}{r}=-\frac{2}{3} \frac{\alpha_{s}}{r} \quad \Rightarrow \quad \kappa_{s}=-\frac{2}{3}
$$

While for quark-antiquark (or for diquark-antidiquark) we have a negative sign in the Coulomb term, but the factor $f_{s}$ (for color singlet configuration) is positive:

$$
f_{1}=+\frac{4}{3} \Rightarrow V_{V}(r)=-\left(+\frac{4}{3}\right) \frac{\alpha_{s}}{r}=-\frac{4}{3} \frac{\alpha_{s}}{r} \Rightarrow \kappa_{s}=-\frac{4}{3}
$$

This section results are listed in the Table 2.1.

Table 2.1: Color Factor for different color configurations.

\begin{tabular}{|r|c|c|r|r|}
\hline sign & $f_{s}$ & $\kappa_{s}$ & structure & color configuration \\
\hline- & $+4 / 3$ & $-4 / 3$ & $q-\bar{q}$ & color singlet \\
- & $-1 / 6$ & $+1 / 6$ & $q-\bar{q}$ & color octet \\
+ & $-2 / 3$ & $-2 / 3$ & $q-q$ & color antitriplet \\
+ & $+1 / 3$ & $+1 / 3$ & $q-q$ & color sextet \\
\hline
\end{tabular}

As an extra information, in the color factor $f_{s}$ calculation we always encounter summations over the product of the Gell-Mann matrices in the form $\lambda_{i j}^{a} \lambda_{k l}^{a}$. We can use a simple formula for this expression (from color Fierz identities, where $n_{c}=3$ is the number of colors):

$$
\sum_{\alpha} \lambda_{i j}^{\alpha} \lambda_{k l}^{\alpha}=2\left(\delta_{i l} \delta_{j k}-\frac{1}{n_{c}} \delta_{i j} \delta_{k l}\right) \Longrightarrow \sum_{\alpha} \lambda_{i j}^{\alpha} \lambda_{k l}^{\alpha}=2 \delta_{i l} \delta_{j k}-\frac{2}{3} \delta_{i j} \delta_{k l}
$$

\subsection{The Coupling Constant $\alpha_{s}$}

The behavior of the $\alpha_{s}\left(\left|q^{2}\right|\right)$ in QCD is more complex than its analogue in QED. We restrict ourselves to present some main results and an example of how to estimate it in the quarkonium context.

As discussed in Refs. [1], the running coupling constant can be expressed as a function of an arbitrary parameter of reference $\mu^{2}$, from the renormalization group equation. In the 1-loop approximation we have:

$$
\alpha_{s}\left(\left|q^{2}\right|\right)=\frac{\alpha_{s}\left(\mu^{2}\right)}{1+\left[\alpha_{s}\left(\mu^{2}\right) / 12 \pi\right]\left(11 n_{c}-2 n_{f}\right) \ln \left(\left|q^{2}\right| / \mu^{2}\right)}, \quad\left(\left|q^{2}\right| \gg \mu^{2}\right)
$$

where $n_{c}=3$ is the number of colors in QCD, $n_{f}$ is the number of active flavors, and it is related with loop corrections in the gluon exchange, as if a virtual pair of flavor up to $n_{f}$ could be created, in the same sense of eletron-positron loops in the one photon exchange in QED. The active flavors are the ones considered light in that scale $\left(m_{q} \ll \mu\right)$, so for chamonium we have $n_{f}=3$, since $u, d$ and $s$ are considered light.

In QED, the case $\mu^{2}=0$ is the large distance behavior, where $\alpha_{e}\left(\mu^{2}\right) \rightarrow \alpha_{e}\left(\left|q^{2}\right|=0\right) \cong$ $1 / 137)$. This is not a good reference point in perturbative QCD, because that is when $\alpha_{s}$ is large (due to color confinement), then perturbation theory is no longer applicable. In order to consider the interaction of only one gluon exchange we must have $\alpha_{s}\left(\mu^{2}\right)<1$. 
A common way of expressing $\alpha_{s}$ is through the introduction of a new variable $\Lambda$, which represents the energy scale of the process:

$$
\ln \left(\Lambda^{2}\right)=\ln \left(\mu^{2}\right)-12 \pi /\left[\left(11 n_{c}-2 n_{f}\right) \alpha_{s}\left(\mu^{2}\right)\right]
$$

Then $\alpha_{s}$ can be expressed in term of the exchanged momentum $\left|q^{2}\right|$ and $\Lambda^{2}$ :

$$
\alpha_{s}\left(\left|q^{2}\right|\right)=\frac{12 \pi}{\left(11 n_{c}-2 n_{f}\right) \ln \left(\left|q^{2}\right| / \Lambda^{2}\right)}, \quad\left(\left|q^{2}\right| \gg \Lambda^{2}\right)
$$

This is the 1-loop expression of the running coupling constant $\alpha_{s}$, but one can find in the literature derivations to higher orders.

The scale constant $\Lambda$ is usually assumed to be around a few hundred $\mathrm{MeVs}$ in quarkonium context. It is common to see in the literature values around $200 \mathrm{MeV}$ for charmonium. Also, there are different ways to estimated the momentum transfer: with a characteristic length scale [64], the kinetic energy [63], constituents masses, etc. One common point of reference is the value of this coupling constant in the energy scale of the boson $Z$. From the PDG [52] we have $m_{Z}=91.1876(21) \mathrm{GeV}$, and $\alpha_{s}\left(m_{Z}\right)=0.1185(6)$. This values could be used to evolve the coupling constant to a desired scale, or to estimate $\Lambda$ and apply the eq. (2.62).

In more sophisticated models of quarkonium, like the relativized potential model of Godfrey and Isgur [68] also used in Ref. [67], the coupling constant $\alpha_{s}$ is considered as a "running" parameter, that changes according to the energy scale of each bound state. But due to the phenomenological character of our model and its minimalist purpose, we will adopt the $\alpha_{s}$ as a constant in the potential, a common approach in many charmonium models.

The value of $\alpha_{s}$ will be obtained from a fitting procedure into the charmonium experimental data, where it is considered as a free parameter, allowed to vary within an acceptable range of values while the $\chi^{2}$ is minimized (in order to find the best set where the difference between model predictions and experimental input is minimal). The charm quark mass $m_{c}$, the string tension $b$, and the gaussian parameter $\sigma$ in model 2, will also be considered as free parameters in the fitting procedure, and once the best set is found, they are kept fixed to generate the whole mass spectrum.

\subsection{Wavefunction at the Origin}

The value of the square modulus of the wavefunction $|\Psi(0)|^{2}$ is an important piece of information, since the spin-spin splitting in model 1 is proportional to it (see Section 3.1), as are other quantities like decay rates which can be related to experimental data.

In these models of quarkonium, only S-wave states $(\ell=0)$ have non-zero value of the wavefunction at the origin. For states with orbital angular momentum $(\ell \neq 0)$, the centrifugal term in Schrödinger equation creates a "centrifugal barrier", which makes the wavefunction at the origin vanish. Thus, for $\ell \neq 0$ we will assume $|\Psi(0)|^{2}=0$ and for S-wave we have:

$$
|\Psi(0)|^{2}=\left|Y_{0}^{0}(\theta, \phi) R_{n, \ell}(0)\right|^{2}=\frac{\left|R_{n, \ell}(0)\right|^{2}}{4 \pi}, \quad \text { for } \quad \ell=0 .
$$

So the important quantity at stage is the square modulus of the radial wavefunction at the origin $\left|R_{n, \ell}(0)\right|^{2}$, which can be obtained from the numerical calculations.

Next we present two methods for obtaining the wavefunction at the origin, one based on the assumptions of the numerical method, that justifies how it vanishes for orbitally excited 
states, and another based on an analytical deduction for S-wave states.

\subsubsection{Generic $|\Psi(0)|^{2}$}

The numerical solution of the Schrödinger equation (see reference [61] for details) is based in the Runge-Kutta method, and the integration of the differential equation starts at the origin and goes on until a critical point is reached (where the program can assume that the wavefunction will asymptotically converge to zero with $r \rightarrow \infty$, ensuring it is normalizable).

In order to estimate its value at the origin, an expansion of the radial wavefunction in power series of $r$ is performed in the Schrödinger equation. Then with the restriction that the potential can't be more divergent at the origin than $1 / r^{2}$, one can take the limit $r \rightarrow 0$ and obtain the following result in terms of the (normalized) reduced radial wavefunction $y_{n, \ell}(r)=r R_{n, \ell}(r)$ :

$$
y_{n, \ell}(\delta \approx 0)=(\delta)^{\ell+1} N_{n, \ell} \quad \Rightarrow \quad\left|R_{n, \ell}(\delta)\right|=\frac{\delta^{\ell+1}}{\delta} N_{n, \ell}=\delta^{\ell} N_{n, \ell}=N_{n, \ell}, \quad \text { for } \quad \ell=0 .
$$

where $\delta$ represents the numerical "zero" (from where the program starts the numerical integration with the Runge-Kutta procedure), and it is defined with the precision chosen as input when one calls the function schroe in the program, that is: $\delta=h / 10$, where a reasonable choice can be $h=0.01$ (input), which is the step size in the numerical integration. The normalization factor of $y_{n, \ell}(r)$ is denoted $N_{n, \ell}$ and it can be calculated (with a numerical integration with Mathematica built-in functions) once we have obtained the function $y_{n, \ell}(r)$ within this method. So it is immediate that for $\ell=0$, whatever may be the value of $\delta$, the radial wavefunction will be exactly the normalization factor $N_{n, \ell}$ of $y_{n, \ell}(r)$. For $\ell \neq 0$ we still get that the wavefunction at the origin is approximately zero, and the bigger the value of $\ell$, or the smaller is the step size $h$, closer to zero it will be (that's why we will assume it is zero within this numerical procedure). Then the result from this analysis is:

$$
\left|R_{n, \ell}(\delta \approx 0)\right|^{2}=N_{n, \ell}^{2}=\left(\int_{x \min =\delta \sim 0}^{x \max \sim \infty}\left[y_{n, \ell}(r)\right]^{2} d r\right)^{-1}, \text { for } \ell=0 .
$$

Notice that the normalization factor is the same for $R_{n, \ell}(r)$ or $y_{n, \ell}(r)$. Just to be clear, the program works with $y_{n, \ell}(r)$ : as the integration of the Runge-Kutta method goes on, the values of $y_{n, \ell}(r)$ are obtained for points in the $r$ coordinate separated by the step size $h$. In the end these points are interpolated using a built-in function of the software Mathematica, and finally one has a continuous function $y_{n, \ell}(r)$, which still needs to be normalized, imposing the condition of eq. (2.3). The number $N_{n, \ell}$, which we denoted by normalization factor, is simply the factor by which we have to multiply $y_{n, \ell}(r)$ to make it a normalized function: $N_{n, \ell} y_{n, \ell}(r)$ is the normalized reduced radial wavefuntion. In the above equation (2.65) $y_{n, \ell}(r)$ is not yet normalized, that's why the inverse of the integral of its square is exactly the square of the normalization factor $N_{n, \ell}$, which happens to be also the value of the square of the radial wavefunction at the origin. In equation (2.64) we explicitly wrote the normalization factor $N_{n, \ell}$, because we said they were already normalized. In the next Section 2.6.2 we will suppress the indices $n, \ell$ for a lighter notation.

\subsubsection{S-wave $|\Psi(0)|^{2}$}

The previous method might seem dubious, but it does indeed yields approximately the same result of the method presented in this section, which is very common in the literature 
of quarkonium models, and whose deduction can be found in Section 5.3 of Ref. [60]. As we show next, one can obtain an interesting formula relating the wavefunction at the origin $|\Psi(0)|^{2}$ and the radial potential $V(r)$, for S-wave states $(\ell=0)$. The deduction begins with the Schrödinger equation in the form:

$$
-\nabla^{2} \Psi=2 \mu(E-V) \Psi
$$

and using the wavefunction for $\ell=0$ (S-wave):

$$
\Psi(\mathbf{r})=Y_{0}^{0}(\theta, \phi) R(r)=\frac{1}{\sqrt{4 \pi}} \frac{y(r)}{r} \Longrightarrow \nabla^{2} \Psi(\mathbf{r})=\frac{1}{\sqrt{4 \pi}} \frac{y^{\prime \prime}(r)}{r}
$$

then inserting eq. (2.67) in eq. (2.66), multiplying both sides by $\frac{y^{\prime}}{r \sqrt{4 \pi}}$ and integrating over all space:

$$
\begin{gathered}
-\int \mathrm{d}^{3} r \frac{y^{\prime \prime} y^{\prime}}{4 \pi r^{2}}=-\frac{1}{2} \int_{0}^{\infty} \mathrm{d} r\left(y^{\prime 2}\right)^{\prime}=-\left.\frac{1}{2}\left(y^{\prime 2}\right)\right|_{0} ^{\infty}=-\left.2 \pi\left(\Psi+r \Psi^{\prime}\right)^{2}\right|_{0} ^{\infty}=2 \pi|\Psi(0)|^{2} \\
=2 \mu \int \mathrm{d}^{3} r[E-V(r)] \frac{y y^{\prime}}{4 \pi r^{2}}=\mu \int_{0}^{\infty} \mathrm{d} r[E-V(r)]\left(y^{2}\right)^{\prime}=\mu \int_{0}^{\infty} \mathrm{d} r V^{\prime}(r)\left(y^{2}\right)=\mu\left\langle V^{\prime}\right\rangle(2)
\end{gathered}
$$

Notice that the "integral by parts trick" was used in the last step.

So for S-wave $(\ell=0)$ :

$$
|\Psi(0)|^{2}=\frac{\mu}{2 \pi}\left\langle V^{\prime}(r)\right\rangle \quad \Longrightarrow \quad|R(0)|^{2}=2 \mu\left\langle V^{\prime}(r)\right\rangle
$$

\subsubsection{Discussion for $|\Psi(0)|^{2}$}

We have seen two different ways to get the value of the square modulus of the radial wavefunction at the origin of the system (in the CM frame). The first one seemed dubious at a first glance, even though it goes quickly to zero when orbital momentum is non-zero, as expected. But with the second way, which is an "analytical" calculation for S-wave states, (therefore it seems more reliable), we usually get approximately the same values we get with the first method, only with a small difference, commonly after a few decimal digits. That difference gets smaller if we increase the precision of the calculations by reducing the step of the numerical integration $h$.

Due to practical reasons, we adopted $h=0.01$ in all calculations. Since the fitting procedure requires a huge number of calculations until it reaches the set of parameters that minimizes $\chi^{2}$, using smaller precision is only viable for single calculations (the fitting procedure is discussed in Chapter 4).

Let us analyse the sources of numerical error in each method:

1) Both have the same error inherent to the method used to obtain the wavefunction $y(r)$, in which there is an error from the Runge-Kutta method itself, as from the built-in functions of Mathematica used to solve the Schrödinger equation: the numerical integration and the interpolation of the points obtained with that method to get the continuous function $y(r)$.

2) - a) In the first method, there is the error from the numerical integration used to calculate the normalization factor square $N^{2}$ - and mind that in $N$ the potential and wavefunction properties are indirectly contained.

$2)$ - b) In the second method there is the error in taking the expectation value of the 
potential derivative, which is also a numerical integration. But to do that we need to use the normalized wavefunction, then the error from calculation $N$ will be contained in the second method. The value $R(\delta)$ might be implicit here too, because the program only gives the wavefunction between $x_{\min }=\delta$ (the "numerical zero") and $x_{\max }$ (the point where the program identifies that the wavefunction has already converged to zero within the precision chosen, so it is considered as the "numerical $\infty$ "). This range is also the region of integration when we calculate any expectation value. But since the second method has an "analytical" deduction to get the formula (2.69), and it is widely adopted in other quarkonium models (for instance, in Ref. [63]) it seems more appropriate to choose it as the standard form to get the wavefunction at the origin.

Withal, we don't have strong reasons to believe the second method is much better than the first one, even though it is surely more elegant. The best way to minimize any numerical error is to increase the precision of the calculations by reducing the step of the numerical integration $h$. In all the calculations performed, both values have always converged when we increased the precision, but with $h=0.01$ they were already close enough and the difference in the results were very small or negligible.

Considering all the discussions and many preliminary calculations, we have chosen to present our final results for S-wave states using the second method. For states with orbital angular momentum, we assumed that the wavefunction at the origin is zero.

Adopting a more sophisticated numerical method, for example Numerov instead of Runge-Kutta (as in Ref. [63]), could increase the precision of the calculations. But the physical assumptions of each model and the parameter set used in the potential affect much more the final results than the choice of the numerical method does.

Our choice, as we checked, is sufficient to reproduce the results of Refs. [63] and [66, 67] within the precision presented in these works (number of digits in the results).

\subsection{The Tetraquark Wavefunction}

\subsubsection{Angular Momentum Coupling}

Here we present the tetraquark wavefunction that we are going to use in the next sections, with emphasis in the angular part (spin and orbital), which is going to be important in the calculation of the tensor spin-dependent perturbative correction.

In the Schrödinger formalism, the time-dependent part of the wavefunction can be factorized as usual, and for our purpouse, the angular and radial parts will also be factorized. But since we are dealing with quarks, the wavefunction also has a flavor and a color part. Since we are aiming at a tetraquark composed only of charm quarks, the flavor part is very simple. The color part was discussed in Section 2.4. So the total wavefunction combines all of these:

$$
\Psi_{\text {total }}=\left\{\psi_{\text {spatial }} \otimes \chi_{\text {spin }} \otimes \phi_{\text {flavor }} \otimes \psi_{\text {color }}\right\} \chi_{\text {time }}
$$

We leave aside the time, flavor and color parts, and look only to the spatial and spin parts. We factorize the radial part from the angular part that combines orbital and spin (which are going to be coupled using Clebsh-Gordan coefficients):

$$
\psi_{\text {spatial }} \otimes \chi_{\text {spin }}=\psi(r)\left\{Y_{\ell}^{m}(\theta, \phi) \otimes \chi\left(s_{1}, s_{2}, s_{3}, s_{4}\right)\right\}
$$

We will use the indices 1 and 2 for the two quarks inside the diquark, and 3 and 4 for the two antiquarks inside the antidiquark (see Fig. 3.1 in Section 3.3). In accordance with our factorization of the 4-body system into three 2-body subsystems, we will build the 
angular part coupling the diquark and antidiquark, which are built coupling the quarks and antiquarks, respectively.

It will also be assumed that the angular part is composed of pure states, where total spin and orbital momentum are good quantum numbers, even though in a more rigourous (relativistic) treatment, only the total angular momentum $J$ would be conserved, and there could be a mixing of different values of $S$ and $\ell$ which can be coupled into the same value of $J$.

First, we introduce the notation for the spin of the diquark (and antidiquark). This is the well-known coupling between two spin $1 / 2$ particles, whose total can be 0 (singlet) or 1 (triplet):

$$
\begin{aligned}
\left|s_{1}, s_{2}, S_{d}\right\rangle & \equiv\left|s_{3}, s_{4}, S_{\bar{d}}\right\rangle \\
|0,0\rangle & =(\uparrow \downarrow-\downarrow \uparrow) / \sqrt{2} \\
|1,1\rangle & =\uparrow \uparrow \\
|1,0\rangle & =(\uparrow \downarrow+\downarrow \uparrow) / \sqrt{2} \\
|1,-1\rangle & =\downarrow \downarrow
\end{aligned}
$$

Always, $s_{1}=s_{2}=s_{3}=s_{4}=1 / 2$. Then $S_{d}$ will denote the total spin of the diquark and $S_{\bar{d}}$ the total for the antidiquark (both always 1 in our choices for the tetraquark). We write the possible couplings in a generic form $\left|S, M_{S}\right\rangle$, where $S$ is a total spin and $M_{S}$ is its $\mathrm{z}$-component. The arrows denote the spins of each constituent, in the order 1,2 for the diquark and 3, 4 for the antidiquark. As usual the arrow up denotes spin up: $\left|\frac{1}{2}, \frac{1}{2}\right\rangle$ and arrow down denotes spin down: $\left|\frac{1}{2},-\frac{1}{2}\right\rangle$.

As discussed in detail Chapter 5, we choose the diquark (composed of same flavor quarks), in the ground state (no orbital nor radial excitations) and in the color antitriplet state, which implies that its total spin has to be 1 in order to respect the Pauli exclusion principle (and the same for the antidiquark). Then, for the coupling of spin 1 diquark and antidiquark, we can have the total tetraquark spin as $S_{T}=0,1,2$.

First we will look only to the total spin wavefunctions, showing the three possible cases of total spin for the tetraquark, coupling the spin 1 diquark and antidiquark (with ClebshGordan coefficients corresponding to the $1 \times 1$ coupling) into the principal case (maximum z-component $M_{S_{T}}=S_{T}$ ). We use the index " 12 " for the diquark and " 34 " for the antidiquark, and we show it in terms of diquark and antidiquark spin basis, and also in terms of the two quarks and two antiquarks spin basis (each group of four arrows is always in the order "1234"):

$$
\begin{aligned}
& \left(S_{d}=1\right) \otimes\left(S_{\bar{d}}=1\right) \longrightarrow\left|S_{T}, M_{S_{T}}\right\rangle=|0,0\rangle_{S_{T}} \\
& =\frac{1}{\sqrt{3}}|1,1\rangle_{12} \otimes|1,-1\rangle_{34}-\frac{1}{\sqrt{3}}|1,0\rangle_{12} \otimes|1,0\rangle_{34}+\frac{1}{\sqrt{3}}|1,-1\rangle_{12} \otimes|1,1\rangle_{34} \\
& =\frac{1}{\sqrt{3}}|\uparrow \uparrow\rangle_{12} \otimes|\downarrow \downarrow\rangle_{34}-\frac{1}{\sqrt{3}}\left|\frac{\uparrow \downarrow+\downarrow \uparrow}{\sqrt{2}}\right\rangle_{12} \otimes\left|\frac{\uparrow \downarrow+\downarrow \uparrow}{\sqrt{2}}\right\rangle_{34}+\frac{1}{\sqrt{3}}|\downarrow \downarrow\rangle_{12} \otimes|\uparrow \uparrow\rangle_{34} \\
& =\frac{1}{\sqrt{12}}(2 \uparrow \uparrow \downarrow \downarrow+2 \downarrow \downarrow \uparrow \uparrow-\uparrow \downarrow \uparrow \downarrow-\uparrow \downarrow \downarrow \uparrow-\downarrow \uparrow \uparrow \downarrow-\downarrow \uparrow \downarrow \uparrow)
\end{aligned}
$$




$$
\begin{aligned}
& \left(S_{d}=1\right) \otimes\left(S_{\bar{d}}=1\right) \longrightarrow\left|S_{T}, M_{S_{T}}\right\rangle=|1,1\rangle_{S_{T}} \\
& =\frac{1}{\sqrt{2}}|1,1\rangle_{12} \otimes|1,0\rangle_{34}-\frac{1}{\sqrt{2}}|1,0\rangle_{12} \otimes|1,1\rangle_{34} \\
& =\frac{1}{\sqrt{2}}|\uparrow \uparrow\rangle_{12} \otimes\left|\frac{\uparrow \downarrow+\downarrow \uparrow}{\sqrt{2}}\right\rangle_{34}-\frac{1}{\sqrt{2}}\left|\frac{\uparrow \downarrow+\downarrow \uparrow}{\sqrt{2}}\right\rangle_{12} \otimes|\uparrow \uparrow\rangle_{34} \\
& =\frac{1}{2}(\uparrow \uparrow \uparrow \downarrow+\uparrow \uparrow \downarrow \uparrow-\uparrow \downarrow \uparrow \uparrow-\downarrow \uparrow \uparrow \uparrow)
\end{aligned}
$$

$$
\begin{aligned}
& \left(S_{d}=1\right) \otimes\left(S_{\bar{d}}=1\right) \longrightarrow\left|S_{T}, M_{S_{T}}\right\rangle=|2,2\rangle_{S_{T}} \\
& =|1,1\rangle_{12} \otimes|1,1\rangle_{34} \\
& =|\uparrow \uparrow\rangle_{12} \otimes|\uparrow \uparrow\rangle_{34} \\
& =\uparrow \uparrow \uparrow \uparrow
\end{aligned}
$$

The other cases with lower z-component $M_{S_{T}}$ can be constructed in the same way. These wavefunctions were inspired in the ones presented in Refs. [69, 72, 73, 74], and we generalized them to include orbital angular momentum between diquark and antidiquark.

Now we show how to couple the total spin wavefunctions to spherical harmonics in order to obtain a specific value of total angular momentum $J_{T}$ in the tetraquark. We start by coupling the total spin of the tetraquark $S_{T}$ with the orbital momentum $L_{T}$ (we will denote this orbital momentum by $L_{T}$ to make it clear that it is the one between the diquark and the antidiquark). We will also show only the principal case (maximum z-component $M_{J_{T}}=J_{T}$ ), which we use to calculate the expectation value of the tensor operator (the direction $\mathrm{z}$ is arbitray for the whole system, so the result should not depend on the z-component $M_{J_{T}}$ ).

For $L_{T}=1$ we have seven possibilities of $J_{T}$ if we are considering spin 1 diquark and antiquark: one for $S_{T}=0\left(J_{T}=1\right)$, three for $S_{T}=1\left(J_{T}=0,1,2\right)$ and three for $S_{T}=2$ $\left(J_{T}=1,2,3\right)$. We start with the notation $\left|S_{T}, M_{S_{T}}\right\rangle_{S_{T}} \otimes\left|L_{T}, M_{L_{T}}\right\rangle_{L_{T}}$ so the use of the Clebsh-Gordan coefficients is clear, then we open it into the diquark and antiquark spin basis, using the previous spin wavefunctions (or others with different z-components when necessary) and the notation $Y_{\ell}^{m}(\theta, \varphi)$ for the spherical harmonics. Finally, in the last step we open it in the quarks and antiquarks spin basis.

First we present the trivial coupling between total spin $S_{T}=0$ that was calculated in eq. (2.73), and orbital momentum $L_{T}=1$ (which implies $J_{T}=1$ ):

$$
\begin{aligned}
& {\left[\left(S_{d}=1\right) \otimes\left(S_{\bar{d}}=1\right) \rightarrow\left(S_{T}=0\right)\right] \otimes\left(L_{T}=1\right) \longrightarrow\left|J_{T}, M_{J_{T}}\right\rangle=|1,1\rangle_{J_{T}}} \\
& =|0,0\rangle_{S_{T}} \otimes|1,1\rangle_{L_{T}} \\
& =\left(\frac{1}{\sqrt{3}}|1,1\rangle_{12} \otimes|1,-1\rangle_{34}-\frac{1}{\sqrt{3}}|1,0\rangle_{12} \otimes|1,0\rangle_{34}+\frac{1}{\sqrt{3}}|1,-1\rangle_{12} \otimes|1,1\rangle_{34}\right) Y_{1}^{1}(\theta, \varphi) \\
& =\left(\frac{1}{\sqrt{12}}(2 \uparrow \uparrow \downarrow \downarrow+2 \downarrow \downarrow \uparrow \uparrow-\uparrow \downarrow \uparrow \downarrow-\uparrow \downarrow \downarrow \uparrow-\downarrow \uparrow \uparrow \downarrow-\downarrow \uparrow \downarrow \uparrow)\right) Y_{1}^{1}(\theta, \varphi)
\end{aligned}
$$


Next, we present one example with total spin $S_{T}=2, L_{T}=1$ and $J_{T}=2$ :

$$
\begin{aligned}
{\left[\left(S_{d}=1\right) \otimes\left(S_{\bar{d}}=1\right) \rightarrow\left(S_{T}=2\right)\right] \otimes\left(L_{T}=1\right) \longrightarrow\left|J_{T}, M_{J_{T}}\right\rangle=|2,2\rangle_{J_{T}} } \\
=\sqrt{\frac{2}{3}}|2,2\rangle_{S_{T}} \otimes|1,0\rangle_{L_{T}}-\frac{1}{\sqrt{3}}|2,1\rangle_{S_{T}} \otimes|1,1\rangle_{L_{T}} \\
=\sqrt{\frac{2}{3}}\left(|1,1\rangle_{12} \otimes|1,1\rangle_{34}\right) Y_{1}^{0}(\theta, \varphi) \\
\quad-\frac{1}{\sqrt{3}}\left(\frac{1}{\sqrt{2}}|1,1\rangle_{12} \otimes|1,0\rangle_{34}+\frac{1}{\sqrt{2}}|1,0\rangle_{12} \otimes|1,1\rangle_{34}\right) Y_{1}^{1}(\theta, \varphi) \\
=\sqrt{\frac{2}{3}}\left(|\uparrow \uparrow\rangle_{12} \otimes|\uparrow \uparrow\rangle_{34}\right) Y_{1}^{0}(\theta, \varphi) \\
-\frac{1}{\sqrt{3}}\left(\frac{1}{\sqrt{2}}|\uparrow \uparrow\rangle_{12} \otimes\left|\frac{\uparrow \downarrow+\downarrow \uparrow}{\sqrt{2}}\right\rangle_{34}+\frac{1}{\sqrt{2}}\left|\frac{\uparrow \downarrow+\downarrow \uparrow}{\sqrt{2}}\right\rangle_{12} \otimes|\uparrow \uparrow\rangle_{34}\right) Y_{1}^{1}(\theta, \varphi) \\
=\sqrt{\frac{2}{3}}(\uparrow \uparrow \uparrow \uparrow) Y_{1}^{0}(\theta, \varphi)-\frac{1}{\sqrt{3}}\left(\frac{1}{2}(\uparrow \uparrow \uparrow \downarrow+\uparrow \uparrow \downarrow \uparrow+\uparrow \downarrow \uparrow \uparrow+\downarrow \uparrow \uparrow \uparrow)\right) Y_{1}^{1}(\theta, \varphi)
\end{aligned}
$$

Notice that it was necessary to make use of another spin state $|2,1\rangle_{S_{T}}$, i. e., a total spin $S_{T}=2$ but with z-component $M_{S_{T}}=1$, which was built with the same procedure, coupling the spin 1 diquark and antidiquark, (then we added the intermediate step to show the change to the spin $1 / 2$ basis).

At last, we present one example with $S_{T}=1, L_{T}=1$ and $J_{T}=0$ (for brevity, we will skip the intermediate step between the spin 1 basis and the spin $1 / 2$ basis):

$$
\begin{aligned}
& {\left[\left(S_{d}=1\right) \otimes\left(S_{\bar{d}}=1\right) \rightarrow\left(S_{T}=1\right)\right] \otimes\left(L_{T}=1\right) \longrightarrow\left|J_{T}, M_{J_{T}}\right\rangle=|0,0\rangle_{J_{T}}} \\
& =\frac{1}{\sqrt{3}}|1,1\rangle_{S_{T}} \otimes|1,-1\rangle_{L_{T}}-\frac{1}{\sqrt{3}}|1,0\rangle_{S_{T}} \otimes|1,0\rangle_{L_{T}}+\frac{1}{\sqrt{3}}|1,-1\rangle_{S_{T}} \otimes|1,1\rangle_{L_{T}} \\
& =\frac{1}{\sqrt{3}}\left(\frac{1}{\sqrt{2}}|1,1\rangle_{12} \otimes|1,0\rangle_{34}-\frac{1}{\sqrt{2}}|1,0\rangle_{12} \otimes|1,1\rangle_{34}\right) Y_{1}^{-1}(\theta, \varphi) \\
& \quad-\frac{1}{\sqrt{3}}\left(\frac{1}{\sqrt{2}}|1,1\rangle_{12} \otimes|1,-1\rangle_{34}-\frac{1}{\sqrt{2}}|1,-1\rangle_{12} \otimes|1,1\rangle_{34}\right) Y_{1}^{0}(\theta, \varphi) \\
& \quad+\frac{1}{\sqrt{3}}\left(\frac{1}{\sqrt{2}}|1,0\rangle_{12} \otimes|1,-1\rangle_{34}-\frac{1}{\sqrt{2}}|1,-1\rangle_{12} \otimes|1,0\rangle_{34}\right) Y_{1}^{1}(\theta, \varphi) \\
& =\frac{1}{\sqrt{3}}\left(\frac{1}{2}(\uparrow \uparrow \uparrow \downarrow+\uparrow \uparrow \downarrow \uparrow-\uparrow \downarrow \uparrow \uparrow-\downarrow \uparrow \uparrow \uparrow)\right) Y_{1}^{-1}(\theta, \varphi) \\
& -\frac{1}{\sqrt{3}}\left(\frac{1}{\sqrt{2}}(\uparrow \uparrow \downarrow \downarrow-\downarrow \downarrow \uparrow \uparrow)\right) Y_{1}^{0}(\theta, \varphi) \\
& \quad+\frac{1}{\sqrt{3}}\left(\frac{1}{2}(\uparrow \downarrow \uparrow \uparrow+\downarrow \uparrow \downarrow \downarrow-\downarrow \downarrow \uparrow \downarrow-\downarrow \downarrow \uparrow)\right) Y_{1}^{1}(\theta, \varphi)
\end{aligned}
$$

This time we made use of other two different total spin 1 wavefunction: the ones with z-components 0 and $-1\left(|1,0\rangle_{S_{T}}\right.$ and $\left.|1,-1\rangle_{S_{T}}\right)$.

These examples can give us an idea of how apparently complicated it can be to take an expectation value of these states. Luckily, for the spin-spin (Sec. 3.1) and spin-orbit (Sec. 3.2) corrections, we don't need to use them since we can obtain the angular factors only from the spin and orbital quantum numbers. But for the tensor (Sec. 3.3), we only have a general result in terms of eigenvalues of the interaction between two spin $1 / 2$ particles (which in our 
case, would be inside the diquark (and inside the antiquark). Then for a proper treatment of the tensor interaction in the diquark-antidiquark system, we will explicitly apply the tensor in this kind of wavefunctions, and show that within our approximations (discussed in detail in Sec. 3.3), it is equivalent to apply the tensor directly in the pair diquark-antidiquark (spin 1 basis) or as a sum of four tensor interactions between each pair of quark-antiquark (spin $1 / 2$ basis).

\subsection{2 $J^{P C}$ Quantum Numbers in the Tetraquark}

As discussed in Refs. [23, 26], we can use the diquark-antidiquark basis to label the possible quantum numbers $J^{P C}$ of the tetraquark. Using the following notation:

$$
\left|T_{4 Q}\right\rangle=\left|S_{d}, S_{\bar{d}}, S_{T}, L_{T}\right\rangle_{J_{T}}
$$

where $S_{d}$ is the total spin of the diquark, $S_{\bar{d}}$ is the total spin of the antidiquark, $S_{T}$ is the total spin of the tetraquark, assumed to come from the coupling $S_{d} \otimes S_{\bar{d}}, L_{T}$ is the orbital angular momentum relative to the diquark-antidiquark system (in the 2-body approximation), and $J_{T}$ is the total angular momentum of the tetraquark, assumed to come from the coupling $S_{T} \otimes L_{T}$.

The general formula for charge-conjugation and parity of the tetraquark can be put as:

$$
\begin{aligned}
& C_{T}=(-1)^{L_{T}+S_{T}} \\
& P_{T}=(-1)^{L_{T}}
\end{aligned}
$$

Since we are interested in the $T_{4 c}$ tetraquark, where the diquarks are composed by two charm quarks (therefore identical fermions), they can only have spin 1 in the antitriplet color configuration (further discussion in Chapter 5). In this case, for the S-wave all-charm tetraquark we have the following possibilities:

$$
\begin{gathered}
\left|0^{++}\right\rangle_{T 4 c}=\left|S_{c c}=1, S_{\bar{c} \bar{c}}=1, S_{T}=0, L_{T}=0\right\rangle_{J_{T}=0} \\
\left|1^{+-}\right\rangle_{T 4 c}=\left|S_{c c}=1, S_{\bar{c} \bar{c}}=1, S_{T}=1, L_{T}=0\right\rangle_{J_{T}=1} \\
\left|2^{++}\right\rangle_{T 4 c}=\left|S_{c c}=1, S_{\bar{c} \bar{c}}=1, S_{T}=2, L_{T}=0\right\rangle_{J_{T}=2}
\end{gathered}
$$

Note that all the S-wave tetraquarks described above have positive parity. The introduction of one orbital excitation will bring a factor $(-1)$ both in parity and in charge conjugation. Then all the $\mathrm{P}$-wave states (with $L_{T}=1$ ) will have odd parity and the opposite charge conjugation in comparison with the S-wave case.

In the Table 2.2 we list the $J^{P C}$ quantum numbers of the 10 possibilities that we have considering the S-wave and $\mathrm{P}$-wave of the all-charm tetraquark built with spin 1 diquarks (also in accordance with Refs. [48] and [32]). 
Table 2.2: Results for the $J^{P C}$ quantum numbers of the $T_{4 c}$ with $\left[S_{d}=S_{\bar{d}}=\right.$ $\left.1 \rightarrow S_{T}=0,1,2\right] \otimes L_{T}=0,1$.

\begin{tabular}{|c|c|c|c|}
\hline \hline$S_{T}$ & $L_{T}$ & $J_{T}$ & $J^{P C}$ \\
\hline 0 & 0 & 0 & $0^{++}$ \\
1 & 0 & 1 & $1^{+-}$ \\
2 & 0 & 2 & $2^{++}$ \\
\hline 0 & 1 & 1 & $1^{--}$ \\
\hline 1 & 1 & 2 & $2^{-+}$ \\
1 & 1 & 1 & $1^{-+}$ \\
1 & 1 & 0 & $0^{-+}$ \\
\hline 2 & 1 & 3 & $3^{--}$ \\
2 & 1 & 2 & $2^{--}$ \\
2 & 1 & 1 & $1^{--}$ \\
\hline \hline
\end{tabular}




\section{Chapter 3}

\section{Spin-Dependent Interactions}

The three spin-dependent terms carry a factor $1 / m^{2}$ (for equal masses), what justifies the treatment with first-order perturbation theory in heavy quarkonium models.

Putting together the three spin-dependent terms (for equal masses) we have:

$$
V_{S p i n}^{(1)}=C_{S S}(r)\left(\mathbf{S}_{\mathbf{1}} \cdot \mathbf{S}_{\mathbf{2}}\right)+C_{L S}(r)(\mathbf{L} \cdot \mathbf{S})+C_{T}(r)\left(\frac{\left(\mathbf{S}_{\mathbf{1}} \cdot \mathbf{r}\right)\left(\mathbf{S}_{\mathbf{2}} \cdot \mathbf{r}\right)}{\mathbf{r}^{2}}-\frac{1}{3}\left(\mathbf{S}_{\mathbf{1}} \cdot \mathbf{S}_{\mathbf{2}}\right)\right)
$$

where the radial-dependent coefficients are obtained from the vector and scalar contributions of the radial potential in eq. (2.13):

$$
V_{V}^{(0)}(r)=\kappa_{s} \frac{\alpha_{s}}{r} \quad \text { and } \quad V_{S}^{(0)}(r)=b r
$$

For equal masses the coefficients are:

$$
\begin{aligned}
C_{S S}(r) & =\frac{2}{3 m^{2}} \nabla^{2} V_{V}(r) \\
C_{L S}(r) & =\frac{1}{2 m^{2}} \frac{1}{r}\left[3 \frac{d V_{V}(r)}{d r}-\frac{d V_{S}(r)}{d r}\right] \\
C_{T}(r) & =\frac{1}{m^{2}}\left[\frac{1}{r} \frac{d V_{V}(r)}{d r}-\frac{d^{2} V_{V}(r)}{d r^{2}}\right]
\end{aligned}
$$

(notice that if introduce a constant term $V_{0}$ in the potential it won't affect the radial coefficients, since only derivatives appear in them).

Just to simplify the number manipulation and operator notation, we redefine the tensor operator with an extra factor 12 , and then divide its coefficient by 12 :

$$
\begin{aligned}
\mathbf{S}_{\mathbf{1 2}} & \equiv 12\left(\frac{\left(\mathbf{S}_{\mathbf{1}} \cdot \mathbf{r}\right)\left(\mathbf{S}_{\mathbf{2}} \cdot \mathbf{r}\right)}{\mathbf{r}^{2}}-\frac{1}{3}\left(\mathbf{S}_{\mathbf{1}} \cdot \mathbf{S}_{\mathbf{2}}\right)\right)=4\left[3\left(\mathbf{S}_{\mathbf{1}} \cdot \hat{\mathbf{r}}\right)\left(\mathbf{S}_{\mathbf{2}} \cdot \hat{\mathbf{r}}\right)-\mathbf{S}_{\mathbf{1}} \cdot \mathbf{S}_{\mathbf{2}}\right] \\
C_{T^{\prime}}(r) & \equiv \frac{1}{12 m^{2}}\left[\frac{1}{r} \frac{d V_{V}(r)}{d r}-\frac{d^{2} V_{V}(r)}{d r^{2}}\right]
\end{aligned}
$$

We will call the expectation value of the operator $\mathbf{S}_{12}$ as "tensor factor", and its calculation will be shown in Section 3.3. Notice that it only depends on the unit vector in the radial direction $\hat{\mathbf{r}}=\mathbf{r} /|\mathbf{r}|$ (in the line joining the position of both particles), and their spins, but not on the distance $r \equiv|\mathbf{r}|$.

The expectation value of all three radial-dependent coefficients will be calculated using 
the wavefunction obtained with the numerical solution of the Schrödinger equation.

The total spin-dependent perturbative correction (in model 1) will be:

$$
\left\langle V_{\text {Spin }}^{(1)}\right\rangle=\left\langle C_{S S}(r)\right\rangle\left\langle\mathbf{S}_{\mathbf{1}} \cdot \mathbf{S}_{\mathbf{2}}\right\rangle+\left\langle C_{L S}(r)\right\rangle\langle\mathbf{L} \cdot \mathbf{S}\rangle+\left\langle C_{T^{\prime}}(r)\right\rangle\left\langle\mathbf{S}_{\mathbf{1 2}}\right\rangle
$$

\section{$3.1 \quad$ Spin-Spin}

The expectation value of the operator of spin-spin interaction can be calculated in terms of the spin quantum numbers using the following relation:

$$
\left\langle\mathbf{S}_{\mathbf{1}} \cdot \mathbf{S}_{\mathbf{2}}\right\rangle=\left\langle\frac{1}{2}\left(\mathbf{S}^{2}-\mathbf{S}_{\mathbf{1}}{ }^{2}-\mathbf{S}_{\mathbf{2}}{ }^{2}\right)\right\rangle=\frac{1}{2}\left[S(S+1)-S_{1}\left(S_{1}+1\right)-S_{2}\left(S_{2}+1\right)\right]
$$

where $S_{1}$ and $S_{2}$ are the spins of particles 1 and 2 respectively, and $S$ is the total spin in consideration. Since each quark (or antiquark) has spin $1 / 2$, they can couple into a singlet $S=0$ and triplet $S=1$. Then the spin-spin term $\left\langle\mathbf{S}_{\mathbf{1}} \cdot \mathbf{S}_{\mathbf{2}}\right\rangle$, according do eq. (3.8) is:

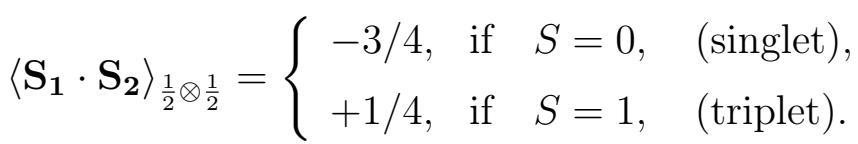

In diquark-antidiquark tetraquarks, the diquark or antidiquark can have spin 0 or 1 . If one or both of them is zero, then $\left\langle\mathbf{S}_{\mathbf{1}} \cdot \mathbf{S}_{\mathbf{2}}\right\rangle=0$ (we could consider a superposition of two states with spins $\left(\left|S_{d}=1, S_{\bar{d}}=0\right\rangle \pm\left|S_{d}=0, S_{\bar{d}}=1\right\rangle\right) / \sqrt{2}$, but the particular case in which we are actually interested is the one where both have spin 1: $S_{d}=1$ and $S_{\bar{d}}=1$.

Then if both diquark and antidiquark have spin 1, they can couple into a singlet $S=0$, triplet $S=1$, and a "pentet" $S=2$. So if we treat the spin-spin interaction based on the coupling of the total spin of each system in a 2-body fashion (with $S_{1}$ and $S_{2}$ as the diquark and antidiquark spins respectively, and $S$ now is the total spin of the tetraquark), then equation (3.8) gives:

$$
\left\langle\mathbf{S}_{\mathbf{1}} \cdot \mathbf{S}_{\mathbf{2}}\right\rangle_{1 \otimes 1}=\left\{\begin{array}{llll}
-2, & \text { if } & S=0, & \text { (singlet) }, \\
-1, & \text { if } \quad S=1, & \text { (triplet) }, \\
+1, & \text { if } S=2, & \text { (pentet). }
\end{array}\right.
$$

For $m_{1}=m_{2} \equiv m$ the radial coefficient of the spin-spin term reads:

$$
\begin{gathered}
C_{S S}(r)=\frac{2}{3 m^{2}} \nabla^{2} V_{V}(r)=\frac{2}{3 m^{2}} \nabla^{2}\left[+\kappa_{s} \frac{\alpha_{s}}{r}\right]=\frac{2}{3 m^{2}} \kappa_{s} \alpha_{s}\left[-4 \pi \delta^{3}(r)\right]=-\frac{8 \pi \kappa_{s} \alpha_{s}}{3 m^{2}} \delta^{3}(r) \\
\left\langle C_{S S}(r)\right\rangle=-\frac{8 \pi \kappa_{s} \alpha_{s}}{3 m^{2}}\left\langle\delta^{3}(r)\right\rangle=-\frac{8 \pi \kappa_{s} \alpha_{s}}{3 m^{2}}|\Psi(0)|^{2}=-\frac{2 \kappa_{s} \alpha_{s}}{3 m^{2}}|R(0)|^{2}
\end{gathered}
$$

The wavefunction at the origin can be calculated as explained in Section 2.6.

Notice that from eq. (3.10) we can see that the spin-spin coupling is stronger for two spin 1 particles (diquark-antidiquark) than for two spin 1/2 (quark-quark, or antiquarkantiquark). Remember that there is still an additional factor 2 due to the color factor $(-2 / 3$ for the color antitriplet diquarks and $-4 / 3$ for the color singlet tetraquarks). 
We can use these results to obtain the zeroth-order potential of model 2, where we include the spin-spin interaction with a gaussian replacing the Dirac delta. When the spin operator $\mathbf{S}_{\mathbf{1}} \cdot \mathbf{S}_{\mathbf{2}}$ acts in the wavefunction, it will produce the constant term of equation (3.8), then we have:

$$
V_{2}^{(0)}(r)=\kappa_{s} \frac{\alpha_{s}}{r}+b r-\frac{8 \pi \kappa_{s} \alpha_{s}}{3 m^{2}}\left(\frac{\sigma}{\sqrt{\pi}}\right)^{3} \mathrm{e}^{-\sigma^{2} r^{2}}\left(\frac{1}{2}\left[S(S+1)-S_{1}\left(S_{1}+1\right)-S_{2}\left(S_{2}+1\right)\right]\right)
$$

\subsection{Spin-Orbit}

The expectation value of the operator of spin-orbit interaction can be calculated in terms of the quantum numbers of total angular momentum $J$ (defined by the vector sum: $\mathbf{J}=\mathbf{L}+\mathbf{S})$, total spin $S$ and orbital angular momentum $\ell$ using the following relation:

$$
\langle\mathbf{L} \cdot \mathbf{S}\rangle=\left\langle\frac{1}{2}\left(\mathbf{J}^{2}-\mathbf{L}^{2}-\mathbf{S}^{2}\right)\right\rangle=\frac{1}{2}[J(J+1)-S(S+1)-\ell(\ell+1)]
$$

For S-wave states $(\ell=0)$, the spin-orbit term $\langle\mathbf{L} \cdot \mathbf{S}\rangle$ is always zero, as we can immediately see from eq. (3.14), if $\ell=0 \Rightarrow J=S$ then $\langle\mathbf{L} \cdot \mathbf{S}\rangle=0$.

Similary, for singlet states $(S=0)$, the spin-orbit term $\langle\mathbf{L} \cdot \mathbf{S}\rangle$ is always zero too, as we can immediately see from eq. (3.14), if $S=0 \Rightarrow J=\ell$ then $\langle\mathbf{L} \cdot \mathbf{S}\rangle=0$.

However, for triplet states $(S=1)$ with $\ell \neq 0$ we have $J=\ell+1, \ell, \ell-1$, which yields:

$$
\langle\mathbf{L} \cdot \mathbf{S}\rangle_{S=1, \ell \neq 0}=\left\{\begin{array}{cl}
\ell, & \text { if } J=\ell+1, \\
-1, & \text { if } J=\ell \\
-(\ell+1), & \text { if } \quad J=\ell-1
\end{array}\right.
$$

When combining spin 1 diquarks and antidiquarks, the total tetraquark spin can be $S=0,1,2$.

Note that for $S=2$, using the formula (3.14) we have a general case if $\ell \geq 2$ :

$$
\langle\mathbf{L} \cdot \mathbf{S}\rangle_{S=2, \ell \geq 2}=\left\{\begin{array}{cl}
2 \ell, & \text { if } \quad J=\ell+2, \\
\ell-2, & \text { if } \quad J=\ell+1, \\
-3, & \text { if } \quad J=\ell, \\
-(\ell+3), & \text { if } \quad J=\ell-1, \\
-2(\ell+1), & \text { if } \quad J=\ell-2 .
\end{array}\right.
$$

In particular, for $\ell=1$ we only have $J=3,2,1$, then:

$$
\langle\mathbf{L} \cdot \mathbf{S}\rangle_{S=2, \ell=1}=\left\{\begin{array}{ccc}
2, & \text { if } & J=3 \\
-1, & \text { if } & J=2 \\
-3, & \text { if } & J=1
\end{array}\right.
$$


For $m_{1}=m_{2} \equiv m$ the radial coefficient of the spin-orbit term reads:

$$
\begin{gathered}
C_{L S}(r)=\frac{1}{2 m^{2}} \frac{1}{r}\left[3 \frac{d V_{V}(r)}{d r}-\frac{d V_{S}(r)}{d r}\right]=\frac{1}{2 m^{2}} \frac{1}{r}\left[3 \frac{d}{d r}\left(+\kappa_{s} \alpha_{s} \frac{1}{r}\right)-\frac{d}{d r}(+b r)\right] \\
=\frac{1}{2 m^{2}} \frac{1}{r}\left[3 \kappa_{s} \alpha_{s}\left(-\frac{1}{r^{2}}\right)-b\right]=\frac{1}{2 m^{2}}\left[-3 \kappa_{s} \alpha_{s} \frac{1}{r^{3}}-\frac{b}{r}\right]=-\frac{3 \kappa_{s} \alpha_{s}}{2 m^{2}} \frac{1}{r^{3}}-\frac{b}{2 m^{2}} \frac{1}{r} \\
\left\langle C_{L S}(r)\right\rangle=-\frac{3 \kappa_{s} \alpha_{s}}{2 m^{2}}\left\langle\frac{1}{r^{3}}\right\rangle-\frac{b}{2 m^{2}}\left\langle\frac{1}{r}\right\rangle
\end{gathered}
$$

\subsection{Tensor}

The spin-dependent term called tensor interaction is not so simple to handle.

The radial coefficient of the tensor term is not a problem, for $m_{1}=m_{2} \equiv m$ it reads:

$$
\begin{aligned}
& C_{T^{\prime}}(r)= \frac{1}{12 m^{2}}\left[\frac{1}{r} \frac{d V_{V}(r)}{d r}-\frac{d^{2} V_{V}(r)}{d r^{2}}\right]=\frac{1}{12 m^{2}}\left[\frac{1}{r} \frac{d}{d r}\left(+\kappa_{s} \alpha_{s} \frac{1}{r}\right)-\frac{d^{2}}{d r^{2}}\left(+\kappa_{s} \alpha_{s} \frac{1}{r}\right)\right] \\
&= \frac{1}{12 m^{2}}\left[\frac{1}{r} \kappa_{s} \alpha_{s}\left(-\frac{1}{r^{2}}\right)-\kappa_{s} \alpha_{s} \frac{d}{d r}\left(-\frac{1}{r^{2}}\right)\right]=\frac{1}{12 m^{2}} \kappa_{s} \alpha_{s}\left[\left(-\frac{1}{r^{3}}\right)-\left(+2 \frac{1}{r^{3}}\right)\right] \\
&= \frac{1}{12 m^{2}} \kappa_{s} \alpha_{s}\left[-3 \frac{1}{r^{3}}\right]=-\frac{\kappa_{s} \alpha_{s}}{4 m^{2}} \frac{1}{r^{3}} \\
&\left\langle C_{T^{\prime}}(r)\right\rangle=-\frac{\kappa_{s} \alpha_{s}}{4 m^{2}}\left\langle\frac{1}{r^{3}}\right\rangle
\end{aligned}
$$

The expectation value of the operator $\mathbf{S}_{\mathbf{1 2}}$ (see eq. 3.6), which we denoted by "tensor factor" $\left(\equiv\left\langle\mathbf{S}_{\mathbf{1 2}}\right\rangle\right)$, requires a more elaborate calculation. The tensor operator $\mathbf{S}_{\mathbf{1 2}}$ carries only spin and orbital dependence. This term doesn't actually depend on the radial distance

This functional form appears in many contexts. For instance, it can be derived from empirical and symmetry arguments commonly associated with the deuteron, regarding the spin and isospin of the proton-neutron bound state, where the tensor potential is considered as part of the Hamiltonian when solving the Schrödinger equation, what causes a S and D waves mixing on the ground state (good references on this subject are the Chapter XIII of the textbook [75], the article [76] and the review [77]). Other important example is the magnetic dipole interaction between proton and electron in the hydrogen atom, which is related to the hyperfine splitting (see, for instance the Section 6.5 of the textbook [78]).

However, in heavy quark interaction, the tensor potential is usually considered as a perturbative correction (just like the spin-spin and spin-orbit). Thus we won't consider any mixings or transitions, but only the expectation value of the tensor operator in a "pure" state with well defined orbital angular momentum and total spin.

The results for the diagonal matrix elements of the tensor operator can be found for example in Ref. [60], and in greater detail in the textbook [79], where the tensor (treated as first-order perturbation) appears in the electron-electron magnetic dipole interaction in the Helium atom. The interaction between the electrons is very similar to the interaction between quarks, since they are also spin $1 / 2$ point particles. 
The expectation value of the tensor between to spin $1 / 2$ particles is non-zero only for:

$$
\begin{aligned}
& \text { 1) } \ell \neq 0 \text { and } S=1 \quad \text { (triplet), } \\
& \text { 2) } J=\ell, \quad \text { or } \quad J=\ell-1, \quad \text { or } \quad J=\ell+1 .
\end{aligned}
$$

Next we will show how to obtain the general result for the interaction of two spin $1 / 2$ particles (following the deduction in the textbook [79]) with algebraic manipulations of the operators in the tensor. We will make use of the the following relation, that can be found in the appendix A.33 about spherical harmonics of the same textbook:

$$
\begin{aligned}
\left\langle(\mathbf{a} \cdot \mathbf{b}) r^{2}-3(\mathbf{a} \cdot \mathbf{r})(\mathbf{b} \cdot \mathbf{r})\right\rangle & =\left\langle\sum_{i, j=1}^{3} a_{i} b_{j}\left(r^{2} \delta_{i j}-3 x_{i} x_{j}\right)\right\rangle \\
& =\frac{-\left\langle r^{2}\right\rangle}{(2 \ell+3)(2 \ell-1)}\left\langle\left[2 \mathbf{L}^{\mathbf{2}}(\mathbf{a} \cdot \mathbf{b})-3(\mathbf{a} \cdot \mathbf{L})(\mathbf{b} \cdot \mathbf{L})-3(\mathbf{b} \cdot \mathbf{L})(\mathbf{a} \cdot \mathbf{L})\right]\right\rangle
\end{aligned}
$$

where $\mathbf{L}$ is the orbital angular momentum operator (vector), $\mathbf{a}$ and $\mathbf{b}$ are any two vectors which commute with $\mathbf{r}, \mathbf{L}$ and each other. We quote the text: "This equation should be considered as a matrix equation in the sense that the matrix elements of the left and right hand side are equal for any transition between two states of the same orbital quantum number $\ell$ (for any value of $\ell$ and for all the combinations of principal and magnetic quantum numbers $\left.n, n^{\prime}, m, m^{\prime}\right) . "$

Comparing the expression above with the tensor operator according to our definition in eq. (3.6), we just need to change the vectors $\mathbf{a}$ and $\mathbf{b}$ by the spins operators $\mathbf{S}_{\mathbf{1}}$ and $\mathbf{S}_{\mathbf{2}}$, watch for the minus sign, the factor 3 in the term with $\mathbf{r}$ (which is a unity vector in the tensor, so this $r^{2}$ cancels), and then we are left with an extra factor 4 :

$$
\mathbf{S}_{\mathbf{1 2}}=\frac{4}{(2 \ell+3)(2 \ell-1)}\left[2 \mathbf{L}^{2}\left(\mathbf{S}_{\mathbf{1}} \cdot \mathbf{S}_{\mathbf{2}}\right)-3\left(\mathbf{S}_{\mathbf{1}} \cdot \mathbf{L}\right)\left(\mathbf{S}_{\mathbf{2}} \cdot \mathbf{L}\right)-3\left(\mathbf{S}_{\mathbf{2}} \cdot \mathbf{L}\right)\left(\mathbf{S}_{\mathbf{1}} \cdot \mathbf{L}\right)\right]
$$

In accordance with the notation of Ref. [79], we will denote by the lower case $\mathbf{s}$ the spin operator of each particle, so $\mathbf{S}_{\mathbf{1}} \rightarrow \mathbf{S}_{\mathbf{1}}$ and $\mathbf{S}_{\mathbf{2}} \rightarrow \mathbf{S}_{\mathbf{2}}$, and $\mathbf{S}$ still denotes the total spin operator. We have chosen to stick to this notation in order to make it clear that some of the relations used hereafter are valid only for spin 1/2 particles (due to the use of anti-commutation relations of the Pauli matrices and eigenvalues that appear), not all of them are necessarily valid for the total spin operator $\mathbf{S}$, or for operators corresponding to the interaction of two spin 1 particles. Then additional care has to be taken when confronting the expectation value of the tensor operator between diquark and antidiquark, since we are interested in the case where both have spin 1 . After presenting the results for two spin $1 / 2$ particles we will discuss the possible approaches in the diquark-antidiquark case. 


\subsubsection{Two spin $1 / 2$ particles}

Taking the expectation value of eq. (3.24) with the new notation we get:

$$
\begin{aligned}
\left\langle\mathbf{S}_{\mathbf{1 2}}\right\rangle & =\frac{4}{(2 \ell+3)(2 \ell-1)}\langle Y\rangle, \\
\text { where } \quad Y & =\left[2 \mathbf{L}^{2}\left(\mathbf{s}_{\mathbf{1}} \cdot \mathbf{s}_{\mathbf{2}}\right)-3\left(\mathbf{s}_{\mathbf{1}} \cdot \mathbf{L}\right)\left(\mathbf{s}_{\mathbf{2}} \cdot \mathbf{L}\right)-3\left(\mathbf{s}_{\mathbf{2}} \cdot \mathbf{L}\right)\left(\mathbf{s}_{\mathbf{1}} \cdot \mathbf{L}\right)\right]
\end{aligned}
$$

Then we just need to find the expectation value of $Y$. Note that we can write:

$$
\left(\mathbf{s}_{\mathbf{1}} \cdot \mathbf{L}\right)\left(\mathbf{s}_{\mathbf{2}} \cdot \mathbf{L}\right)+\left(\mathbf{s}_{\mathbf{2}} \cdot \mathbf{L}\right)\left(\mathbf{s}_{\mathbf{1}} \cdot \mathbf{L}\right)=(\mathbf{S} \cdot \mathbf{L})^{2}-\left(\mathbf{s}_{\mathbf{1}} \cdot \mathbf{L}\right)^{2}-\left(\mathbf{s}_{\mathbf{2}} \cdot \mathbf{L}\right)^{2}
$$

and make use of the following relations:

$$
\begin{gathered}
\mathbf{L} \times \mathbf{L}=i \mathbf{L}, \quad \mathbf{s} \times \mathbf{s}=i \mathbf{s} \\
\left\{s_{i}, s_{j}\right\}=s_{i} s_{j}+s_{j} s_{i}=\frac{1}{2} \delta_{i j} \mathbb{1}_{2}, \quad i, j=x, y, z . \\
(\vec{\sigma} \cdot \mathbf{c})(\vec{\sigma} \cdot \mathbf{d})=(\mathbf{c} \cdot \mathbf{d})+i \vec{\sigma} \cdot(\mathbf{c} \times \mathbf{d})
\end{gathered}
$$

where $\vec{\sigma}$ is the vector of the Pauli matrices $\left(\sigma_{x}, \sigma_{y}, \sigma_{z}\right)$.

Taking $\mathbf{c}=\mathbf{d}=\mathbf{L}$, and multiplying by a factor $1 / 4$ on both sides, we find:

$$
\begin{aligned}
\left(\frac{1}{2} \vec{\sigma} \cdot \mathbf{L}\right)\left(\frac{1}{2} \vec{\sigma} \cdot \mathbf{L}\right) & =\frac{1}{4}(\mathbf{L} \times \mathbf{L})+\frac{1}{2} i\left(\frac{1}{2} \vec{\sigma}\right) \cdot(\mathbf{L} \times \mathbf{L}) \\
(\mathbf{s} \cdot \mathbf{L})^{2} & =\frac{1}{4} \mathbf{L}^{2}+\frac{i}{2}(\mathbf{s}) \cdot(i \mathbf{L}) \\
(\mathbf{s} \cdot \mathbf{L})^{2} & =\frac{1}{4} \mathbf{L}^{2}-\frac{1}{2}(\mathbf{s} \cdot \mathbf{L})
\end{aligned}
$$

Therefore

$$
\left(\mathbf{s}_{\mathbf{1}} \cdot \mathbf{L}\right)^{2}=\frac{1}{4} \mathbf{L}^{2}-\frac{1}{2}\left(\mathbf{s}_{\mathbf{1}} \cdot \mathbf{L}\right) \quad \text { and } \quad\left(\mathbf{s}_{\mathbf{2}} \cdot \mathbf{L}\right)^{2}=\frac{1}{4} \mathbf{L}^{2}-\frac{1}{2}\left(\mathbf{s}_{\mathbf{1}} \cdot \mathbf{L}\right)
$$

Using the relations (3.26), (3.31), and noting that $\mathbf{S}=\mathbf{s}_{\mathbf{1}}+\mathbf{s}_{\mathbf{2}}$, we can rewrite the expression for $Y$ in eq. (3.25) eliminating the unpleasant scalar products between the particle spin operators and orbital operators $\left(\mathbf{s}_{\mathbf{1}} \cdot \mathbf{L}\right.$ and $\left.\mathbf{s}_{\mathbf{2}} \cdot \mathbf{L}\right)$, leaving only terms whose expectation values can be written in terms of the eigenvalues of spin and orbital momentum (as we used in the spin-spin and spin-orbit operators):

$$
Y=\left(2\left(\mathbf{s}_{\mathbf{1}} \cdot \mathbf{s}_{\mathbf{2}}\right)+\frac{3}{2}\right) \mathbf{L}^{2}-\frac{3}{2} \mathbf{S} \cdot \mathbf{L}-3(\mathbf{S} \cdot \mathbf{L})^{2}
$$

We can still use equation (3.8) to put the expression above in terms of the total spin operator:

$$
Y=\mathbf{S}^{2} \mathbf{L}^{2}-\frac{3}{2} \mathbf{S} \cdot \mathbf{L}-3(\mathbf{S} \cdot \mathbf{L})^{2}
$$


Then the expectation value can be calculated just like in the spin-spin and spin-orbit corrections:

$$
\begin{aligned}
\langle Y\rangle & =[S(S+1)][\ell(\ell+1)]-\frac{3}{2}\left(\frac{1}{2}[J(J+1)-S(S+1)-\ell(\ell+1)]\right) \\
& -3\left(\frac{1}{2}[J(J+1)-S(S+1)-\ell(\ell+1)]\right)^{2}
\end{aligned}
$$

Now the tensor factor is a straightforward calculation. Putting everything together we get the following results for the general cases that obey the conditions (3.22) for nonvanishing expectation value of the tensor operator (notice that $\langle Y\rangle$ does vanish if $\ell=0$ or $S=0$ ):

$$
\left\langle\mathbf{S}_{\mathbf{1 2}}\right\rangle_{\frac{1}{2} \otimes \frac{1}{2} \rightarrow S=1, \ell \neq 0}=\left\{\begin{array}{ccc}
-\frac{2 \ell}{(2 \ell+3)}, & \text { if } & J=\ell+1, \\
+2, & \text { if } & J=\ell, \\
-\frac{2(\ell+1)}{(2 \ell-1)}, & \text { if } & J=\ell-1 .
\end{array}\right.
$$

for any of the allowed values of $J$ and $\ell$. For instance, for $\ell=1$ we have $\left\langle\mathbf{S}_{\mathbf{1 2}}\right\rangle=-\frac{2}{5},+2,-4$, for $J=2,1,0$, respectively.

Remember that these results are for diagonal matrix elements, which means the same quantum numbers $S, \ell, J$ in the "bra" and in the "ket": $\left\langle S, \ell, J\left|\mathbf{S}_{\mathbf{1 2}}\right| S, \ell, J\right\rangle$. The tensor actually has non-vanishing non-diagonal matrix elements, but as a first-order perturbation correction they are negligible. They would be important if the tensor were to be used as part of the potential, what would cause the mixing of the wavefunction itself, as in the deuteron model.

\subsubsection{Two spin 1 particles}

Now there is an important point to be noticed here. In this "algebraic" aproach to obtain these three general cases of non-vanishing diagonal elements of the tensor factor for two spin $1 / 2$ particles, we have made use of a few relations that are valid only for Pauli matrices (spin $1 / 2$ ), like equation (3.28). Therefore we can not use this results in the diquark-antidiquark tensor interaction (if we wish to treat it as a 2-body problem), since the diquarks can have spin 0 or 1.

In order to deal with the generalization of the tensor interaction in the tetraquark, we will rewrite the tensor in a form that allows us to get the same results we already know for the particular case of two spin $1 / 2$ particles (which we will verify explicitly for a simple case), and can also be used as a generalization for more complicated cases as the spin 1 diquarks.

The operator $\mathbf{S}_{\mathbf{1 2}}$ is a "rank-2" tensor that can be written in terms of spin operators and spherical harmonics corresponding to $\ell=2$, as presented in the textbook [80], where the tensor also appears as the interaction between the magnetic dipoles of two spin $1 / 2$ particles.

We begin with the tensor operator in the form presented in equation (3.6):

$$
\mathbf{S}_{\mathbf{1 2}}=4\left[3\left(\mathbf{S}_{\mathbf{1}} \cdot \hat{\mathbf{r}}\right)\left(\mathbf{S}_{\mathbf{2}} \cdot \hat{\mathbf{r}}\right)-\mathbf{S}_{\mathbf{1}} \cdot \mathbf{S}_{\mathbf{2}}\right]
$$

We emphasize that the following deduction (inspired in the one presented in Ref. [80]) 
does not use any particular relation or eigenvalues for spin $1 / 2$ particles, only general properties of angular momentum elementary theory. The next step is to write the unity vector $\hat{\mathbf{r}}$ in spherical coordinates and the spin operators in cartesian components:

$$
\begin{aligned}
\mathbf{S}_{\mathbf{1 2}}= & 4\left\{3\left[S_{1 z} \cos \theta+\sin \theta\left(S_{1 x} \cos \varphi+S_{1 y} \sin \varphi\right)\right]\left[S_{2 z} \cos \theta+\sin \theta\left(S_{2 x} \cos \varphi+S_{2 y} \sin \varphi\right)\right]\right. \\
& \left.-\left(S_{1 x} S_{2 x}+S_{1 y} S_{2 y}+S_{1 z} S_{2 z}\right)\right\}
\end{aligned}
$$

We can rearrange it with raising, lowering and z-component spin operators:

$$
\begin{aligned}
S_{ \pm} & =S_{x} \pm i S_{y} \\
S_{ \pm}\left|S, M_{S}\right\rangle & =C_{ \pm}\left(S, M_{S}\right)\left|S, M_{S} \pm 1\right\rangle \\
C_{ \pm}\left(S, M_{S}\right) & =\hbar \sqrt{S(S+1)-M_{S}\left(M_{S} \pm 1\right)} \\
S_{z}\left|S, M_{S}\right\rangle & =\hbar M_{S}\left|S, M_{S}\right\rangle
\end{aligned}
$$
Then:

This is valid for any value of spin! (Actually, for any angular momentum in general).

$$
\begin{aligned}
\mathbf{S}_{\mathbf{1 2}}= & 4\left\{3\left[S_{1 z} \cos \theta+\frac{1}{2} \sin \theta\left(S_{1+} \mathrm{e}^{-i \varphi}+S_{1-} \mathrm{e}^{i \varphi}\right)\right]\right. \\
& \times\left[S_{2 z} \cos \theta+\frac{1}{2} \sin \theta\left(S_{2+} \mathrm{e}^{-i \varphi}+S_{2-} \mathrm{e}^{i \varphi}\right)\right] \\
& \left.-\left(S_{1 x} S_{2 x}+S_{1 y} S_{2 y}+S_{1 z} S_{2 z}\right)\right\}
\end{aligned}
$$

Multiplying and regrouping everything we get six terms:

$$
\mathbf{S}_{12}=4\left[T_{0}+T_{0}^{\prime}+T_{1}+T_{-1}+T_{2}+T_{-2}\right]
$$

where the spherical harmonics corresponding to $\ell=2$ become evident (apart from constants):

$$
\begin{aligned}
T_{0} & =\left(3 \cos ^{2} \theta-1\right) S_{1 z} S_{2 z} \\
T_{0}^{\prime} & =-\frac{1}{4}\left(3 \cos ^{2} \theta-1\right)\left(S_{1+} S_{2-}+S_{1-} S_{2+}\right) \\
T_{1} & =\frac{3}{2} \sin \theta \cos \theta \mathrm{e}^{-i \varphi}\left(S_{1 z} S_{2+}+S_{1+} S_{2 z}\right) \\
T_{-1} & =\frac{3}{2} \sin \theta \cos \theta \mathrm{e}^{i \varphi}\left(S_{1 z} S_{2-}+S_{1-} S_{2 z}\right) \\
T_{2} & =\frac{3}{4} \sin ^{2} \theta \mathrm{e}^{-2 i \varphi} S_{1+} S_{2+} \\
T_{-2} & =\frac{3}{4} \sin ^{2} \theta \mathrm{e}^{2 i \varphi} S_{1-} S_{2-}
\end{aligned}
$$


If we compare it with the $\ell=2$ spherical harmonics, where $Y_{\ell}^{-m}=(-1)^{m} Y_{\ell}^{m *}$ :

$$
\begin{aligned}
Y_{2}^{0} & =\sqrt{\frac{5}{4 \pi}}\left(\frac{3}{2} \cos ^{2} \theta-\frac{1}{2}\right) \\
Y_{2}^{-1} & =+\sqrt{\frac{15}{8 \pi}}(\sin \theta \cos \theta) \mathrm{e}^{-i \varphi} \\
Y_{2}^{1} & =-\sqrt{\frac{15}{8 \pi}}(\sin \theta \cos \theta) \mathrm{e}^{i \varphi} \\
Y_{2}^{-2} & =\frac{1}{4} \sqrt{\frac{15}{2 \pi}} \sin ^{2} \theta \mathrm{e}^{-2 i \varphi} \\
Y_{2}^{2} & =\frac{1}{4} \sqrt{\frac{15}{2 \pi}} \sin ^{2} \theta \mathrm{e}^{2 i \varphi}
\end{aligned}
$$

We can write the six operators in terms of the harmonics and spin operators:

$$
\begin{aligned}
T_{0} & =2 \sqrt{\frac{4 \pi}{5}} Y_{2}^{0} S_{1 z} S_{2 z} \\
T_{0}^{\prime} & =-\frac{1}{4} 2 \sqrt{\frac{4 \pi}{5}} Y_{2}^{0}\left(S_{1+} S_{2-}+S_{1-} S_{2+}\right) \\
T_{1} & =\frac{3}{2} \sqrt{\frac{8 \pi}{15}} Y_{2}^{-1}\left(S_{1 z} S_{2+}+S_{1+} S_{2 z}\right) \\
T_{-1} & =-\frac{3}{2} \sqrt{\frac{8 \pi}{15}} Y_{2}^{1}\left(S_{1 z} S_{2-}+S_{1-} S_{2 z}\right) \\
T_{2} & =3 \sqrt{\frac{2 \pi}{15}} Y_{2}^{-2} S_{1+} S_{2+} \\
T_{-2} & =3 \sqrt{\frac{2 \pi}{15}} Y_{2}^{2} S_{1-} S_{2-}
\end{aligned}
$$

When calculating the expectation value of the tensor operator, we can use the selection rules from the spherical harmonics:

$$
\int Y_{\ell^{\prime}}^{m^{\prime} *}(\theta, \varphi) Y_{2}^{q}(\theta, \varphi) Y_{\ell}^{m}(\theta, \varphi) \mathrm{d} \Omega
$$

where $(\mathrm{d} \Omega=\sin \theta \mathrm{d} \theta \mathrm{d} \varphi)$, and the selection rules can be written as:

$$
\begin{aligned}
\ell^{\prime} & =\ell, \ell-2, \ell+2 \\
m^{\prime} & =m+q
\end{aligned}
$$

It is also convenient to use the following general result in terms of Wigner-3j coefficients for the integrals with the spherical harmonics:

$$
\begin{gathered}
\int Y_{\ell_{1}}^{m_{1}}(\theta, \varphi) Y_{\ell_{2}}^{m_{2}}(\theta, \varphi) Y_{\ell_{3}}^{m_{3}}(\theta, \varphi) \mathrm{d} \Omega= \\
=\sqrt{\frac{\left(2 \ell_{1}+1\right)\left(2 \ell_{2}+1\right)\left(2 \ell_{3}+1\right)}{4 \pi}}\left(\begin{array}{ccc}
\ell_{1} & \ell_{2} & \ell_{3} \\
0 & 0 & 0
\end{array}\right)\left(\begin{array}{ccc}
\ell_{1} & \ell_{2} & \ell_{3} \\
m_{1} & m_{2} & m_{3}
\end{array}\right)
\end{gathered}
$$


Where we have used a calculator ${ }^{1}$ (Ref. [81]) to obtain the Wigner-3j coefficients in eq. $(3.47)$.

This representation of the tensor operator allows us to obtain the same results of eq. (3.35) (the diagonal matrix elements of the tensor between two spin $1 / 2$ particles), that were previously calculated using algebraic manipulation of the spin and orbital momentum operators, where the anti-commutation relation and eigenvalues of the Pauli matrices were necessary, what restricted those results to only systems composed of two spin $1 / 2$ particles. The great advantage of this new representation is that it can be used directly in the spin 1 diquarks if one considers the spin operators $\mathbf{S}_{\mathbf{1}}$ and $\mathbf{S}_{\mathbf{2}}$ as the diquark and antidiquark total spin operators (which will be denoted by $\mathbf{S}_{\mathbf{d}}$ and $\mathbf{S}_{\overline{\mathbf{d}}}$ ), and also the 2-body approximation where the diquarks are considered as point-like structures.

Next we will present three important examples to justify our approach of the tensor in the tetraquark: first we will verify (in one simple case) that we do indeed get the same result for two spin $1 / 2$ particles. Then we will show one complicated approach in the tetraquark (with a not so simple, but also not so long example) using the tetraquark wavefunction in terms of each quark and antiquark spin (as presented in Section 2.7), and considering that the tensor interaction between diquark and antidiquark is given by the sum of the four possible interactions (see Fig. 3.1) between quark-antiquark pairs inside the tetraquark (therefore we will have four interactions between two spin $1 / 2$ particles). Finally, we are going to show that exactly the same result can be found if one looks only to the diquark and antidiquark total spins (within the approximations regarding the distances in this 2-body factorization of the 4-body problem). To keep it short, we are going to show only one example of each and then present the general results used in our numerical calculations.

We start with the simplest case of two spin $1 / 2$ particles (for example the interaction between quark and antiquark bound as a meson) in a triplet state $S=1$ and orbital momentum $L=1$ coupled into a total angular momentum $J=2$ in the principal case. The coupling is a trivial $1 \times 1$ case (we will use a "notation abuse" in order to make the distinction between spin and spherical harmonics very clear):

$$
\begin{aligned}
& {\left[\left(s_{1}=1 / 2\right) \otimes\left(s_{2}=1 / 2\right) \rightarrow S=1\right] \otimes(L=1) \longrightarrow\left|J, M_{J}\right\rangle=|2,2\rangle_{J}} \\
& =|1,1\rangle_{S} \otimes|1,1\rangle_{L} \\
& =\left|\uparrow \uparrow Y_{1}^{1}(\theta, \varphi)\right\rangle
\end{aligned}
$$

According to equation (3.35) we should obtain that the expectation value of the tensor operator of this state (the diagonal matrix element) should be the case of $J=\ell+1$ :

$$
\left\langle\uparrow \uparrow Y_{1}^{1}(\theta, \varphi)\left|\mathbf{S}_{12}\right| Y_{1}^{1}(\theta, \varphi) \uparrow \uparrow\right\rangle=-\frac{2 \ell}{(2 \ell+3)}=-\frac{2}{5}
$$

Then taking the expectation value of the tensor operator in the form of equation (3.40):

$$
\begin{aligned}
& \left\langle\uparrow \uparrow Y_{1}^{1}(\theta, \varphi)\left|\mathbf{S}_{\mathbf{1 2}}\right| Y_{1}^{1}(\theta, \varphi) \uparrow \uparrow\right\rangle= \\
& =\left\langle\uparrow \uparrow Y_{1}^{1}(\theta, \varphi)\left|4\left[T_{0}+T_{0}^{\prime}+T_{1}+T_{-1}+T_{2}+T_{-2}\right]\right| Y_{1}^{1}(\theta, \varphi) \uparrow \uparrow\right\rangle \\
& =\left\langle\uparrow \uparrow Y_{1}^{1}(\theta, \varphi)\left|4\left[T_{0}+T_{0}^{\prime}\right]\right| \uparrow \uparrow Y_{1}^{1}(\theta, \varphi)\right\rangle
\end{aligned}
$$

\footnotetext{
${ }^{1}$ http://www-stone.ch.cam.ac.uk/wigner.shtml
} 
Four matrix elements have vanished due to the selections rules in equations (3.44) and $(3.45)$ :

$$
\begin{aligned}
& \int Y_{1}^{1 *}(\theta, \varphi) Y_{2}^{q}(\theta, \varphi) Y_{1}^{1}(\theta, \varphi) \mathrm{d} \Omega \\
\left(\ell^{\prime}=1\right)= & (\ell=1) \\
\left(m^{\prime}=1\right)= & (m+q=1+q) \Rightarrow q=0 \Rightarrow \text { only } T_{0} \text { and } \mathrm{T}_{0}^{\prime}
\end{aligned}
$$

Notice that in equation (3.43) only $T_{0}$ and $T_{0}^{\prime}$ are proportional to $Y_{2}^{0}(\theta, \varphi)$, so these two terms are the only ones that do not vanish because of the integral with spherical harmonics (but they could still vanish because of the spin).

First we will calculate the orbital integral, which is common to $T_{0}$ and $T_{0}^{\prime}$, using the general result given in terms of the Wigner-3j coefficients in eq. (3.47). Notice that:

$$
Y_{\ell}^{-m}=(-1)^{m} Y_{\ell}^{m *} \Longrightarrow Y_{1}^{1 *}=-Y_{1}^{-1}
$$

Then to compare eq. (3.51) with eq. (3.46) we change $Y_{1}^{1 *}$ to $-Y_{1}^{-1}$ and we get:

$$
\begin{aligned}
& \int Y_{1}^{1 *}(\theta, \varphi) Y_{2}^{0}(\theta, \varphi) Y_{1}^{1}(\theta, \varphi) \mathrm{d} \Omega \\
& =-\int Y_{1}^{-1}(\theta, \varphi) Y_{2}^{0}(\theta, \varphi) Y_{1}^{1}(\theta, \varphi) \mathrm{d} \Omega \\
& =-\sqrt{\frac{(3)(5)(3)}{4 \pi}}\left(\begin{array}{lll}
1 & 2 & 1 \\
0 & 0 & 0
\end{array}\right)\left(\begin{array}{ccc}
1 & 2 & 1 \\
-1 & 0 & 1
\end{array}\right) \\
& =-\sqrt{\frac{(3)(5)(3)}{4 \pi}}\left(\sqrt{\frac{2}{15}}\right)\left(\sqrt{\frac{1}{30}}\right) \\
& =-\frac{1}{5} \sqrt{\frac{5}{4 \pi}}
\end{aligned}
$$

Notice that we used equation (3.47) with $\ell_{1}=1, \ell_{2}=2, \ell_{3}=1$ and $m_{1}=-1, m_{2}=$ $0, m_{3}=1$.

Let's save this result for now and analyse how $T_{0}$ and $T_{0}^{\prime}$ act in the spin part. According to eq. (3.41) we have (using $\hbar=1$ and the orthonormality relation $\langle\uparrow \mid \uparrow\rangle=1$ ):

$$
\begin{aligned}
& T_{0} \sim S_{1 z} S_{2 z} \Longrightarrow\left\langle T_{0}\right\rangle \sim\left\langle\uparrow \uparrow\left|S_{1 z} S_{2 z}\right| \uparrow \uparrow\right\rangle \\
& =\left\langle\uparrow\left|S_{1 z}\right| \uparrow\right\rangle\left\langle\uparrow\left|S_{2 z}\right| \uparrow\right\rangle \\
& =\left(+\frac{\hbar}{2}\right)\langle\uparrow \mid \uparrow\rangle_{1}\left(+\frac{\hbar}{2}\right)\langle\uparrow \mid \uparrow\rangle_{2}=\frac{1}{4}
\end{aligned}
$$

But for $T_{0}^{\prime}$ the raising operators acting in $|\uparrow \uparrow\rangle$ and also the orthogonality of $\langle\uparrow \mid \downarrow\rangle$ leads to zero:

$$
\begin{aligned}
& T_{0}^{\prime} \sim\left(S_{1+} S_{2-}+S_{1-} S_{2+}\right) \Longrightarrow\left\langle T_{0}^{\prime}\right\rangle \sim\left\langle\uparrow \uparrow\left|S_{1+} S_{2-}\right| \uparrow \uparrow\right\rangle+\left\langle\uparrow \uparrow\left|S_{1-} S_{2+}\right| \uparrow \uparrow\right\rangle \\
& =\left\langle\uparrow\left|S_{1+}\right| \uparrow\right\rangle\left\langle\uparrow\left|S_{2-}\right| \uparrow\right\rangle+\left\langle\uparrow\left|S_{1-}\right| \uparrow\right\rangle\left\langle\uparrow\left|S_{2+}\right| \uparrow\right\rangle \\
& =(0)_{1} \hbar\langle\uparrow \mid \downarrow\rangle_{2}+\hbar\langle\uparrow \mid \downarrow\rangle_{1}(0)_{2}=(0)_{1}(0)_{2}+(0)_{1}(0)_{2}=0
\end{aligned}
$$


Then finally, we can compute the final result, remembering the factor 4 in equation (3.40) and the factor $2 \sqrt{\frac{4 \pi}{5}}$ from equation (3.43) that relates $T_{0}$ with $Y_{2}^{0}(\theta, \varphi)$; and of course, the orbital and spin values we calculated in eqs. (3.54) and (3.55):

$$
\begin{aligned}
& \left\langle\uparrow \uparrow Y_{1}^{1}(\theta, \varphi)\left|\mathbf{S}_{12}\right| Y_{1}^{1}(\theta, \varphi) \uparrow \uparrow\right\rangle= \\
& =\left\langle\uparrow \uparrow Y_{1}^{1}(\theta, \varphi)\left|4\left[T_{0}\right]\right| \uparrow \uparrow Y_{1}^{1}(\theta, \varphi)\right\rangle \\
& =\left\langle\uparrow \uparrow Y_{1}^{1}(\theta, \varphi)\left|4\left(2 \sqrt{\frac{4 \pi}{5}} Y_{2}^{0} S_{1 z} S_{2 z}\right)\right| \uparrow \uparrow Y_{1}^{1}(\theta, \varphi)\right\rangle \\
& =4 \times\left(2 \sqrt{\frac{4 \pi}{5}}\right) \times\left(-\frac{1}{5} \sqrt{\frac{5}{4 \pi}}\right) \times\left(\frac{1}{4}\right) \\
& =-\frac{2}{5}
\end{aligned}
$$

We do indeed get the same result that we had calculated in eq. (3.49). Actually, it was already proven that this procedure should yield the same value, since what we did was only to manipulate the exact same tensor into a different form, and we already had the general results for the expectation value of this operator when applied to two spin $1 / 2$ particles coupled into $S=1$ with $\ell \neq 0$ in eq. (3.35). This was an example to illustrate how we do the calculation within this spherical harmonic representation of the tensor operator.

Now comes the tough calculation for the tetraquark, where we are confronted with a four fermion wavefunction coupled to one orbital momentum (only one because we are considering just orbital momentum relative to the diquark-antidiquark system, with zero orbital excitations inside the diquark or the antidiquark, since they are assumed to be compact building blocks of the tetraquark - it could be more complicated if we would include orbital motion inside them, but then the factorization of the 4-body system into three 2-body problems would be an unjustified approximation).

Before we start our calculations for the tetraquark, we shall discuss the two different approaches we thought on how to satisfactorily apply the tensor in the diquark-antidiquark system. As we will further discuss in Chapter 5, the same flavor diquark we will consider has total spin 1 (and so does the antidiquark). Thus the total spin of the tetraquark is a coupling $1 \times 1$ which can result in $S_{T}=0,1,2$. To this total spin we will couple an orbital momentum $L_{T}$ into a specific value of total angular momentum $J_{T}$. Trying to deviate the minimum from the case of two spin $1 / 2$ particles, where the tensor is a well-known interaction, we first thought that it would be appropriate to consider the tensor between every pair $q-\bar{q}$ as indicated in Figure 3.1.

The interaction between the two quarks (whose indices are 1 and 2) inside the diquark is taken into account during the calculation of the 2-body problem to find the mass of the diquark (and since we consider diquarks only in S-wave state, only the spin-spin interaction is relevant; the spin-orbit and tensor are identically zero, as we already discussed). Similary is the interaction between the two antiquarks (whose indices are 3 and 4 ) inside the antidiquark. Then we are left with four possibilities between $q-\bar{q}$ : 1-4, 1-3, 2-4, 2-3. Since every calculation regarding the tetraquark is done considering the approximation of the diquark and antidiquark as point-like structures (in a 2-body problem), from where we obtain the tetraquark radial wavefunction, it is appropriate to consider that the radial-dependence of the tensor term is the same for this four $q-\bar{q}$ interactions, and that it can be obtained using the radial wavefunction with equation (3.21). 
Therefore, all we have to do is to find the angular-dependent (spin and orbital) part, which we denoted by tensor factor, the expectation value of the tensor operator in eq. (3.6). Then the tensor factor for the tetraquark would be a sum of four tensor operators between the four $q-\bar{q}$ pairs: 1-4, 1-3, 2-4, 2-3, applied to the four-spin wavefunctions as we showed in Section 2.7. Using some "notation abuse", we could even obtain this result considering that:

$$
\begin{aligned}
& \mathrm{S}_{\mathrm{d}}=\mathrm{S}_{1}+\mathrm{S}_{2} \\
& \mathrm{~S}_{\overline{\mathrm{d}}}=\mathrm{S}_{3}+\mathrm{S}_{4}
\end{aligned}
$$

Then the tensor between diquark-antidiquark would be:

$$
\begin{gathered}
\mathbf{S}_{\mathbf{d}-\overline{\mathbf{d}}}=12\left(\frac{\left(\mathbf{S}_{\mathbf{d}} \cdot \mathbf{r}\right)\left(\mathbf{S}_{\overline{\mathbf{d}}} \cdot \mathbf{r}\right)}{\mathbf{r}^{2}}-\frac{1}{3}\left(\mathbf{S}_{\mathbf{d}} \cdot \mathbf{S}_{\overline{\mathbf{d}}}\right)\right) \\
\mathbf{S}_{\mathbf{d}-\overline{\mathbf{d}}}=12\left(\frac{\left[\left(\mathbf{S}_{\mathbf{1}}+\mathbf{S}_{\mathbf{2}}\right) \cdot \mathbf{r}\right]\left[\left(\mathbf{S}_{\mathbf{3}}+\mathbf{S}_{\mathbf{4}}\right) \cdot \mathbf{r}\right]}{\mathbf{r}^{2}}-\frac{1}{3}\left[\left(\mathbf{S}_{\mathbf{1}}+\mathbf{S}_{\mathbf{2}}\right) \cdot\left(\mathbf{S}_{\mathbf{3}}+\mathbf{S}_{\mathbf{4}}\right)\right]\right)
\end{gathered}
$$

Making the distribution of the scalar products and regrouping it we get:

$$
\begin{aligned}
\mathbf{S}_{\mathbf{d}-\overline{\mathbf{d}}} & =12\left(\frac{\left(\mathbf{S}_{\mathbf{1}} \cdot \mathbf{r}\right)\left(\mathbf{S}_{\mathbf{4}} \cdot \mathbf{r}\right)}{\mathbf{r}^{2}}-\frac{1}{3}\left(\mathbf{S}_{\mathbf{1}} \cdot \mathbf{S}_{\mathbf{4}}\right)\right)+12\left(\frac{\left(\mathbf{S}_{\mathbf{1}} \cdot \mathbf{r}\right)\left(\mathbf{S}_{\mathbf{3}} \cdot \mathbf{r}\right)}{\mathbf{r}^{2}}-\frac{1}{3}\left(\mathbf{S}_{\mathbf{1}} \cdot \mathbf{S}_{\mathbf{3}}\right)\right) \\
& +12\left(\frac{\left(\mathbf{S}_{\mathbf{2}} \cdot \mathbf{r}\right)\left(\mathbf{S}_{\mathbf{4}} \cdot \mathbf{r}\right)}{\mathbf{r}^{2}}-\frac{1}{3}\left(\mathbf{S}_{\mathbf{2}} \cdot \mathbf{S}_{\mathbf{4}}\right)\right)+12\left(\frac{\left(\mathbf{S}_{\mathbf{2}} \cdot \mathbf{r}\right)\left(\mathbf{S}_{\mathbf{3}} \cdot \mathbf{r}\right)}{\mathbf{r}^{2}}-\frac{1}{3}\left(\mathbf{S}_{\mathbf{2}} \cdot \mathbf{S}_{\mathbf{3}}\right)\right) \\
& =\mathbf{S}_{\mathbf{1 4}}+\mathbf{S}_{\mathbf{1 3}}+\mathbf{S}_{\mathbf{2 4}}+\mathbf{S}_{\mathbf{2 3}}
\end{aligned}
$$
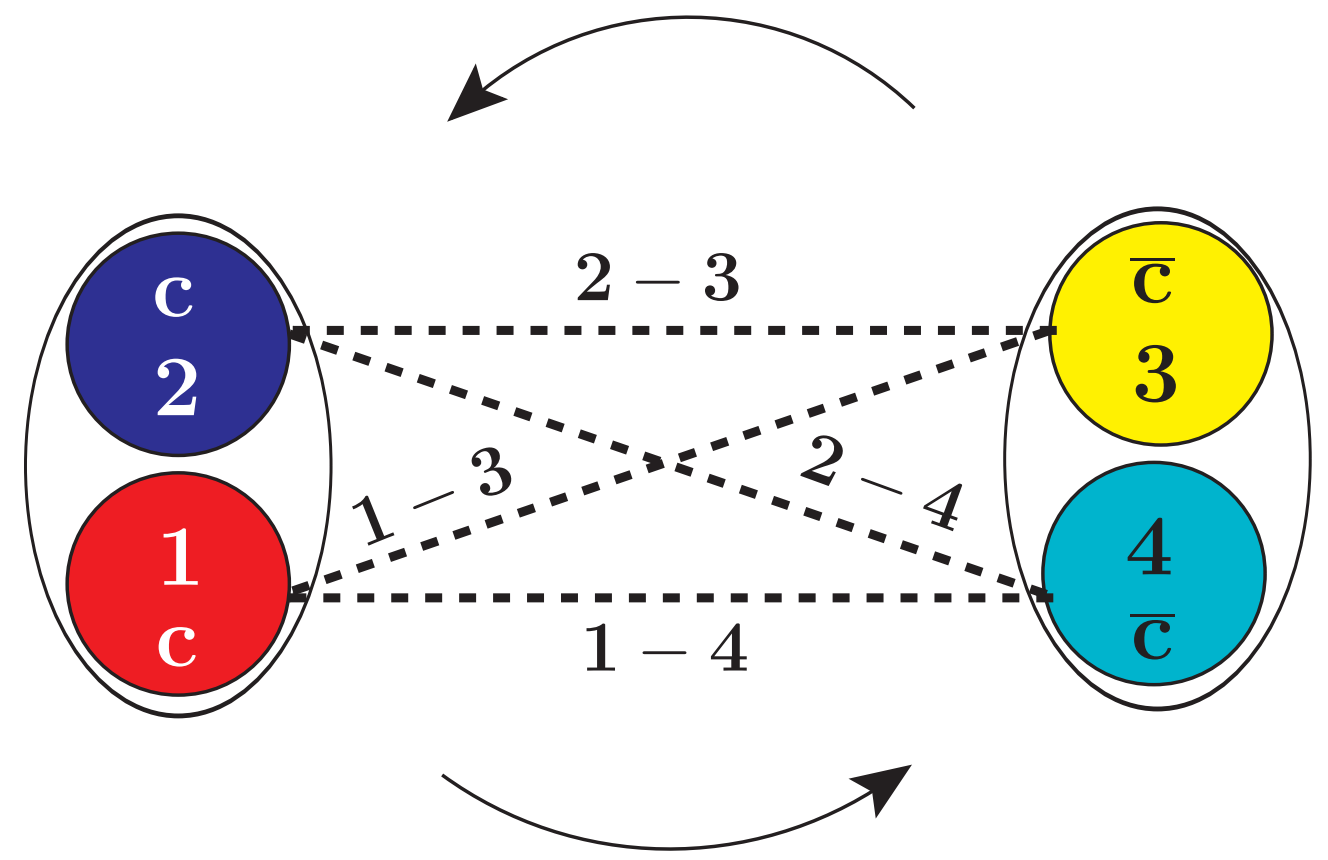

Figure 3.1: Pictorial representation of the tensor interaction between diquark and antidiquark, when considering the interaction between $q-\bar{q}$ pairs. 
Using this approach we need to apply these four tensor operators in the wavefunctions containing the spin of the four particles. In order to give a good view of this approach, we will choose as example the state we showed in equation (2.77): $\mid S_{d}=1, S_{\bar{d}}=1, S_{T}=2, L_{T}=$ $\left.1, J_{T}=2, M_{J_{T}}=2\right\rangle$, which is not too simple nor too long. Then the tensor factor for this tetraquark state would be the expectation value of the sum of the four tensors above, where each one acts in a $q-\bar{q}$ pair (two spin $1 / 2$ particles). The first one will be:

$$
\begin{aligned}
\left\langle\mathbf{S}_{\mathbf{1 4}}\right\rangle & =\left(\sqrt{\frac{2}{3}}\right)^{2}\left\langle\uparrow \uparrow \uparrow \uparrow Y_{1}^{0}(\theta, \varphi)\left|\mathbf{S}_{\mathbf{1 4}}\right| Y_{1}^{0}(\theta, \varphi) \uparrow \uparrow \uparrow \uparrow\right\rangle+ \\
& +\left(-\frac{1}{\sqrt{3}}\right)^{2}\left\langle\left(\frac{\uparrow \uparrow \uparrow \downarrow+\uparrow \uparrow \downarrow \uparrow+\uparrow \downarrow \uparrow \uparrow+\downarrow \uparrow \uparrow \uparrow}{2}\right) Y_{1}^{1}(\theta, \varphi)\left|\mathbf{S}_{\mathbf{1 4}}\right| Y_{1}^{1}(\theta, \varphi) \times\right. \\
& \left.\times\left(\frac{\uparrow \uparrow \uparrow \downarrow+\uparrow \uparrow \downarrow \uparrow+\uparrow \downarrow \uparrow \uparrow+\downarrow \uparrow \uparrow \uparrow}{2}\right)\right\rangle \\
& +\left(\sqrt{\frac{2}{3}}\right)\left(-\frac{1}{\sqrt{3}}\right)\left\langle\uparrow \uparrow \uparrow \uparrow Y_{1}^{0}(\theta, \varphi)\left|\mathbf{S}_{\mathbf{1 4}}\right| Y_{1}^{1}(\theta, \varphi)\left(\frac{\uparrow \uparrow \uparrow \downarrow+\uparrow \uparrow \downarrow \uparrow+\uparrow \downarrow \uparrow \uparrow+\downarrow \uparrow \uparrow \uparrow}{2}\right)\right\rangle \\
& +\left(-\frac{1}{\sqrt{3}}\right)\left(\sqrt{\frac{2}{3}}\right)\left\langle\left(\frac{\left(\frac{\uparrow}{}\right)+\uparrow \uparrow \downarrow \uparrow+\uparrow \downarrow \uparrow \uparrow+\downarrow \uparrow \uparrow \uparrow}{2}\right) Y_{1}^{1}(\theta, \varphi)\left|\mathbf{S}_{\mathbf{1 4}}\right| Y_{1}^{0}(\theta, \varphi) \mid \uparrow \uparrow \uparrow \uparrow\right\rangle
\end{aligned}
$$

We got four terms, two "diagonal" and two "crossed". This is just the tensor $\mathbf{S}_{\mathbf{1 4}}$ which will act only in the quark "1" and antiquark "4". For the other three tensors: $\mathbf{S}_{\mathbf{1 3}}, \mathbf{S}_{\mathbf{2 4}}$ and $\mathbf{S}_{\mathbf{2 3}}$ we will have exactly the same (each one acting in the respective $q-\bar{q}$ pair), and due to the symmetry of the wavefunction all of them will lay the same result, then the total tensor interaction in this approach turns out to be four times the value for one $q-\bar{q}$ pair. This equality can be verified by checking that permutating the spins to get the other three tensor factors doesn't change the wavefunction.

We will show explicitly (to keep it short) only the calculation of the last term, which has an orbital integral where only the term proportional to $T_{-1}$ survives (due to the selection rules):

$$
\begin{gathered}
\int Y_{1}^{1 *}(\theta, \varphi) Y_{2}^{q}(\theta, \varphi) Y_{1}^{0}(\theta, \varphi) \mathrm{d} \Omega \\
\qquad \begin{aligned}
\left(\ell^{\prime}=1\right) & =(\ell=1) \\
\left(m^{\prime}=1\right) & =(m+q=0+q) \Rightarrow q=1 \Rightarrow \text { only } T_{-1}, \\
\text { where } T_{-1} & =-\frac{3}{2} \sqrt{\frac{8 \pi}{15}} Y_{2}^{1}\left(S_{1 z} S_{4-}+S_{1-} S_{4 z}\right)
\end{aligned}
\end{gathered}
$$

Notice we changed the index to "1" and "4" according to our notation for $\mathbf{S}_{\mathbf{1 4}}$. 
The orbital integral will be:

$$
\begin{aligned}
& \int Y_{1}^{1 *}(\theta, \varphi) Y_{2}^{1}(\theta, \varphi) Y_{1}^{0}(\theta, \varphi) \mathrm{d} \Omega \\
& =-\int Y_{1}^{-1}(\theta, \varphi) Y_{2}^{1}(\theta, \varphi) Y_{1}^{0}(\theta, \varphi) \mathrm{d} \Omega \\
& =-\sqrt{\frac{(3)(5)(3)}{4 \pi}}\left(\begin{array}{lll}
1 & 2 & 1 \\
0 & 0 & 0
\end{array}\right)\left(\begin{array}{ccc}
1 & 2 & 1 \\
-1 & 1 & 0
\end{array}\right) \\
& =-\sqrt{\frac{(3)(5)(3)}{4 \pi}}\left(\sqrt{\frac{2}{15}}\right)\left(-\sqrt{\frac{1}{10}}\right) \\
& =+\frac{3 \sqrt{2}}{\sqrt{8 \pi} \sqrt{15}}
\end{aligned}
$$

The spin operator from $T_{-1}$ acting in the state $|\uparrow \uparrow \uparrow \uparrow\rangle,($ using $\hbar=1)$ will result in :

$$
\begin{aligned}
\left(S_{1 z} S_{4-}+S_{1-} S_{4 z}\right)|\uparrow \uparrow \uparrow \uparrow\rangle & \left.=\left|\left(S_{1 z} \uparrow\right) \uparrow \uparrow\left(S_{4-} \uparrow\right)\right\rangle+\left(S_{1-} \uparrow\right) \uparrow \uparrow\left(S_{4 z} \uparrow\right)\right\rangle \\
& \left.=\left|\left(+\frac{\hbar}{2} \uparrow\right) \uparrow \uparrow(\hbar \downarrow)\right\rangle+(\hbar \downarrow) \uparrow \uparrow\left(+\frac{\hbar}{2} \uparrow\right)\right\rangle \\
& =\left|\frac{(\uparrow \uparrow \uparrow \downarrow+\downarrow \uparrow \uparrow \uparrow)}{2}\right\rangle
\end{aligned}
$$

Then when we come with the spin part of the "bra" that was attached to this last term, only two of them survive:

$$
\begin{aligned}
& \left\langle\left(\frac{\uparrow \uparrow \downarrow+\uparrow \uparrow \downarrow \uparrow+\uparrow \downarrow \uparrow \uparrow+\downarrow \uparrow \uparrow \uparrow}{2}\right)\left|\left(S_{1 z} S_{4-}+S_{1-} S_{4 z}\right)\right| \uparrow \uparrow \uparrow \uparrow\right\rangle= \\
& =\left\langle\left(\frac{\uparrow \uparrow \uparrow \downarrow+\uparrow \uparrow \downarrow \uparrow+\uparrow \downarrow \uparrow \uparrow+\downarrow \uparrow \uparrow \uparrow}{2}\right) \mid \frac{(\uparrow \uparrow \uparrow \downarrow+\downarrow \uparrow \uparrow \uparrow)}{2}\right\rangle \\
& =\frac{1}{4}\{[\langle\uparrow \uparrow \uparrow \downarrow \mid \uparrow \uparrow \uparrow \downarrow\rangle+\langle\uparrow \uparrow \downarrow \uparrow \mid \uparrow \uparrow \uparrow \downarrow\rangle+\langle\uparrow \downarrow \uparrow \uparrow \mid \uparrow \uparrow \uparrow \downarrow\rangle+\langle\downarrow \uparrow \uparrow \uparrow \mid \uparrow \uparrow \uparrow \downarrow\rangle] \\
& +[\langle\uparrow \uparrow \uparrow \downarrow \mid \downarrow \uparrow \uparrow \uparrow\rangle+\langle\uparrow \uparrow \downarrow \uparrow \mid \downarrow \uparrow \uparrow \uparrow\rangle+\langle\uparrow \downarrow \uparrow \uparrow \mid \downarrow \uparrow \uparrow \uparrow\rangle+\langle\downarrow \uparrow \uparrow \uparrow \mid \downarrow \uparrow \uparrow \uparrow\rangle]\} \\
& =\frac{1}{4}\{[1+0+0+0]+[0+0+0+1]\} \\
& =\frac{1}{2}
\end{aligned}
$$

Finally, we can pick up everything we calculated for the last of the four terms from the expectation value of the operator $\mathbf{S}_{\mathbf{1 4}}$ acting in the current state: the Clebsh-Gordan coefficients (from the multiplication when taking the expectation value), the coefficient of the operator $T_{-1}$, the results from the orbital integral and the spin operators, and that factor 4 in eq. (3.40):

$$
\begin{aligned}
\text { last term of }\left\langle\mathbf{S}_{\mathbf{1 4}}\right\rangle & =4 \times\left(-\frac{1}{\sqrt{3}}\right)\left(\sqrt{\frac{2}{3}}\right) \times\left(-\frac{3}{2} \sqrt{\frac{8 \pi}{15}}\right) \times\left(+\frac{3 \sqrt{2}}{\sqrt{8 \pi} \sqrt{15}}\right) \times\left(\frac{1}{2}\right) \\
& =\frac{2}{5}
\end{aligned}
$$

Remember that this was only the last term of $\left\langle\mathbf{S}_{\mathbf{1 4}}\right\rangle$. There are still three more to go, 
and then the same 4 terms for each of the others three tensor factors $\left\langle\mathbf{S}_{\mathbf{1 3}}\right\rangle,\left\langle\mathbf{S}_{\mathbf{2 4}}\right\rangle$ and $\left\langle\mathbf{S}_{\mathbf{2 3}}\right\rangle$ ! But luckily, due to the symmetry of the wavefunction, they give the same result.

By following the same procedure, we get that the other "crossed" term (the third) of $\left\langle\mathbf{S}_{\mathbf{1 4}}\right\rangle$ is also $\frac{2}{5}$ and the "diagonal" ones are $\frac{8}{15}$ and $\frac{1}{15}$, respectively (the first and second). Then multiplying the sum of these terms by 4 to account also for the other three $q-\bar{q}$ pairs (1-3, 2-4 and 2-3), we finally have the result for the tensor factor in this particular state, eq. (2.77), of tetraquark:

$$
\begin{aligned}
& \text { For } \quad\left|S_{d}=1, S_{\bar{d}}=1, S_{T}=2, L_{T}=1, J_{T}=2, M_{J_{T}}=2\right\rangle: \\
& \left\langle\mathbf{S}_{\mathbf{1 4}}+\mathbf{S}_{\mathbf{1 3}}+\mathbf{S}_{\mathbf{2 4}}+\mathbf{S}_{\mathbf{2 3}}\right\rangle \equiv 4 \times\left\langle\mathbf{S}_{\mathbf{1 4}}\right\rangle=4 \times\left(\frac{8}{15}+\frac{1}{15}+\frac{2}{5}+\frac{2}{5}\right)=\frac{28}{5}
\end{aligned}
$$

Now we will show that if instead of looking to the four spin $1 / 2$ constituents of the tetraquark in this exhaustively long calculation with four tensors, we can look to the spin 1 diquark and antidiquark with only one "effective tensor" $\mathbf{S}_{\mathbf{d}-\overline{\mathbf{d}}}$ between them, and obtain the exactly same result. The only difference is that we now have to use the proper coefficients for spin 1 when applying the raising, lowering and z-component spin operators. Using equations (3.38) we have:

$$
\begin{aligned}
S_{ \pm}|1,0\rangle & =C_{ \pm}(1,0)|1, \pm 1\rangle \\
\text { where } C_{ \pm}(1,0) & =\hbar \sqrt{1(1+1)-0(0 \pm 1)}=\hbar \sqrt{2} \\
S_{+}|1,-1\rangle & =\hbar \sqrt{1(1+1)-(-1)[(-1)+1]}|1,0\rangle=\hbar \sqrt{2}|1,0\rangle \\
S_{-}|1,1\rangle & =\hbar \sqrt{1(1+1)-1(1-1)}|1,0\rangle=\hbar \sqrt{2}|1,0\rangle
\end{aligned}
$$

Then the same state (see eq. (2.77))in terms of the diquark and antidiquark spins reads:

$$
\begin{aligned}
& \left|S_{d}=1, S_{\bar{d}}=1, S_{T}=2, L_{T}=1, J_{T}=2, M_{J_{T}}=2\right\rangle= \\
& =\sqrt{\frac{2}{3}}\left(|1,1\rangle_{d}|1,1\rangle_{\bar{d}}\right) Y_{1}^{0}(\theta, \varphi)-\frac{1}{\sqrt{3}}\left(\frac{|1,1\rangle_{d}|1,0\rangle_{\bar{d}}+|1,0\rangle_{d}|1,1\rangle_{\bar{d}}}{\sqrt{2}}\right) Y_{1}^{1}(\theta, \varphi)
\end{aligned}
$$

Where we have simplified the notation for the spin using $d$ and $\bar{d}$ as indices.

Just like we did to calculate the expectation value of $\mathbf{S}_{\mathbf{1 4}}$, we will obtain the same four terms, which written in terms of the spin 1 functions is:

$$
\begin{aligned}
& \left\langle\mathbf{S}_{\mathbf{d}-\overline{\mathbf{d}}}\right\rangle=\left(\sqrt{\frac{2}{3}}\right)^{2}\left\langle 1,\left.1\right|_{d}\left\langle 1,\left.1\right|_{\bar{d}} Y_{1}^{0}(\theta, \varphi)\left|\mathbf{S}_{\mathbf{d}-\overline{\mathbf{d}}}\right| Y_{1}^{0}(\theta, \varphi) \mid 1,1\right\rangle_{d} \mid 1,1\right\rangle_{\bar{d}} \\
& +\left(-\frac{1}{\sqrt{3}}\right)^{2}\left(\frac{\left\langle 1,\left.1\right|_{d}\left\langle 1,\left.0\right|_{\bar{d}}+\left\langle 1,\left.0\right|_{d}\left\langle 1,\left.1\right|_{\bar{d}}\right.\right.\right.\right.}{\sqrt{2}}\right) Y_{1}^{1}(\theta, \varphi)\left|\mathbf{S}_{\mathbf{d}-\overline{\mathbf{d}}}\right| Y_{1}^{1}(\theta, \varphi) \times \\
& \times\left(\frac{|1,1\rangle_{d}|1,0\rangle_{\bar{d}}+|1,0\rangle_{d}|1,1\rangle_{\bar{d}}}{\sqrt{2}}\right) \\
& +\left(\sqrt{\frac{2}{3}}\right)\left(-\frac{1}{\sqrt{3}}\right)\left\langle 1,\left.1\right|_{d}\left\langle 1,\left.1\right|_{\bar{d}} Y_{1}^{0}(\theta, \varphi)\left|\mathbf{S}_{\mathbf{d}-\overline{\mathbf{d}}}\right| Y_{1}^{1}(\theta, \varphi)\left(\frac{|1,1\rangle_{d}|1,0\rangle_{\bar{d}}+|1,0\rangle_{d}|1,1\rangle_{\bar{d}}}{\sqrt{2}}\right)\right.\right. \\
& +\left(-\frac{1}{\sqrt{3}}\right)\left(\sqrt{\frac{2}{3}}\right)\left(\frac{\left\langle 1,\left.1\right|_{d}\left\langle 1,\left.0\right|_{\bar{d}}+\left\langle 1,\left.0\right|_{d}\left\langle 1,\left.1\right|_{\bar{d}}\right.\right.\right.\right.}{\sqrt{2}}\right) Y_{1}^{1}(\theta, \varphi)\left|\mathbf{S}_{\mathbf{d}-\overline{\mathbf{d}}}\right| Y_{1}^{0}(\theta, \varphi)|1,1\rangle_{d}|1,1\rangle_{\bar{d}}
\end{aligned}
$$


Again, we will show only the calculation of the last term, analogous to the last term we have calculated for $\left\langle\mathbf{S}_{\mathbf{1 4}}\right\rangle$. But since in this approach we only have one tensor that implicitly accounts for the four $q-\bar{q}$ pairs, we should obtain $4 \times \frac{2}{5}=\frac{8}{5}$. The orbital part will be exactly the same, leading to the operator $T_{-1}$. So we only have to calculate the spin part (again, $\hbar=1$ ):

$$
\begin{aligned}
\left(S_{d z} S_{\bar{d}-}+S_{d-} S_{\overline{d z}}\right)|1,1\rangle_{d}|1,1\rangle_{\bar{d}} & =S_{d z} S_{\bar{d}-}|1,1\rangle_{d}|1,1\rangle_{\bar{d}}+S_{d-} S_{\overline{d z}}|1,1\rangle_{d}|1,1\rangle_{\bar{d}} \\
& =\left(S_{d z}|1,1\rangle_{d}\right)\left(S_{\bar{d}-}|1,1\rangle_{\bar{d}}\right)+\left(S_{d-}|1,1\rangle_{d}\right)\left(S_{\overline{d z}}|1,1\rangle_{\bar{d}}\right) \\
& =\left(+1 \hbar|1,1\rangle_{d}\right)\left(\hbar \sqrt{2}|1,0\rangle_{\bar{d}}\right)+\left(\hbar \sqrt{2}|1,0\rangle_{d}\right)\left(+1 \hbar|1,1\rangle_{\bar{d}}\right) \\
& =\sqrt{2}\left(|1,1\rangle_{d}|1,0\rangle_{\bar{d}}+|1,0\rangle_{d}|1,1\rangle_{\bar{d}}\right)
\end{aligned}
$$

Then when we come with the spin part of the "bra" that was attached to this last term, we get the same result of the previous calculation with the spin part in terms of four spins $1 / 2$ (actually 4 times it, because when we calculated the spin part for $\mathbf{S}_{\mathbf{1 4}}$ we were calculating $1 / 4$ of the total tensor, (remember that in the end we multiplied everything by 4 , to account for the four $q-\bar{q}$ pairs):

$$
\begin{aligned}
& \left.\left(\frac{\left\langle 1,\left.1\right|_{d}\left\langle 1,\left.0\right|_{\bar{d}}+\left\langle 1,\left.0\right|_{d}\left\langle 1,\left.1\right|_{\bar{d}}\right.\right.\right.\right.}{\sqrt{2}}\right)\left|\left(S_{d z} S_{\bar{d}-}+S_{d-} S_{\bar{d} z}\right)\right| 1,1\right\rangle_{d}|1,1\rangle_{\bar{d}}= \\
& =\left(\frac{\left\langle 1,\left.1\right|_{d}\left\langle 1,\left.0\right|_{\bar{d}}+\left\langle 1,\left.0\right|_{d}\left\langle 1,\left.1\right|_{\bar{d}}\right.\right.\right.\right.}{\sqrt{2}}\right) \sqrt{2}\left(|1,1\rangle_{d}|1,0\rangle_{\bar{d}}+|1,0\rangle_{d}|1,1\rangle_{\bar{d}}\right) \\
& =\left\langle 1,\left.1\right|_{d} \mid 1,1\right\rangle_{d}\left\langle 1,\left.0\right|_{\bar{d}} \mid 1,0\right\rangle_{\bar{d}}+\left\langle 1,\left.1\right|_{d} \mid 1,0\right\rangle_{d}\left\langle 1,\left.0\right|_{\bar{d}} \mid 1,1\right\rangle_{\bar{d}} \\
& +\left\langle 1,\left.0\right|_{d} \mid 1,1\right\rangle_{d}\left\langle 1,\left.1\right|_{\bar{d}} \mid 1,0\right\rangle_{\bar{d}}+\left\langle 1,\left.0\right|_{d} \mid 1,0\right\rangle_{d}\left\langle 1,\left.1\right|_{\bar{d}} \mid 1,1\right\rangle_{\bar{d}} \\
& =1+0+0+1=2 \\
& \equiv 4 \times \frac{1}{2}
\end{aligned}
$$

All the other steps of the calculation are the same, the only difference is that in this approach we only have one tensor instead of four, then the result already has a factor 4 implicitly:

$$
\text { last term of } \begin{aligned}
\left\langle\mathbf{S}_{\mathbf{d}-\overline{\mathbf{d}}}\right\rangle & =4 \times\left(-\frac{1}{\sqrt{3}}\right)\left(\sqrt{\frac{2}{3}}\right) \times\left(-\frac{3}{2} \sqrt{\frac{8 \pi}{15}}\right) \times\left(+\frac{3 \sqrt{2}}{\sqrt{8 \pi} \sqrt{15}}\right) \times\left(4 \times \frac{1}{2}\right) \\
& =4 \times \frac{2}{5}=\frac{8}{5}
\end{aligned}
$$

Remember that the first factor 4 above is part of the tensor (see eq. (3.40)). Accordingly, all the other four terms of the tensor in this state (whose calculation we won't show, for brevity) will have an extra factor 4 in the spin part. So in the end we don't multiply it by 4 , of course, since the tensor $\mathbf{S}_{\mathbf{d}-\overline{\mathbf{d}}}$ already describes (implicitly) the interaction between the four $q-\bar{q}$ pairs: 


$$
\begin{gathered}
\text { For } \quad\left|S_{d}=1, S_{\bar{d}}=1, S_{T}=2, L_{T}=1, J_{T}=2, M_{J_{T}}=2\right\rangle: \\
\begin{aligned}
\left\langle\mathbf{S}_{\mathbf{d}-\overline{\mathbf{d}}}\right\rangle & =\left(\frac{32}{15}+\frac{4}{15}+\frac{8}{5}+\frac{8}{5}\right)=\frac{28}{5} \\
& \equiv 4 \times\left(\frac{8}{15}+\frac{1}{15}+\frac{2}{5}+\frac{2}{5}\right)=\frac{28}{5}
\end{aligned}
\end{gathered}
$$

We hope to have explained everything in the clearest way possible.

The results of the orbital integrals for $\ell^{\prime}=\ell=1$ in the form of equation (3.44) are compiled in Table 3.1. Notice that $q$ satisfies the z-component condition of eq. (3.45), that tells which operators $T$ in eq. (3.43) are non-vanishing due to this selection rule.

Table 3.1: Results for the orbital integrals with $\ell^{\prime}=\ell=1$.

\begin{tabular}{|c|c|c|c|c|}
\hline $\mathrm{m}$ & $\mathrm{q}$ & $\mathrm{m}$ & $\int Y_{\ell^{\prime}}^{m^{\prime} *}(\theta, \varphi) Y_{2}^{q}(\theta, \varphi) Y_{\ell}^{m}(\theta, \varphi) \mathrm{d} \Omega$ & Operator \\
\hline 1 & 0 & 1 & $-1 / \sqrt{20 \pi}$ & $T_{0}$ and $T_{0}^{\prime}$ \\
\hline-1 & 0 & -1 & $-1 / \sqrt{20 \pi}$ & $T_{0}$ and $T_{0}^{\prime}$ \\
\hline 0 & 0 & 0 & $+1 / \sqrt{5 \pi}$ & $T_{0}$ and $T_{0}^{\prime}$ \\
\hline 1 & 2 & -1 & $-\sqrt{3 / 10 \pi}$ & $T_{-2}$ \\
\hline-1 & -2 & 1 & $-\sqrt{3 / 10 \pi}$ & $T_{2}$ \\
\hline 1 & 1 & 0 & $+\sqrt{3 / 20 \pi}$ & $T_{-1}$ \\
\hline-1 & -1 & 0 & $+\sqrt{3 / 20 \pi}$ & $T_{1}$ \\
\hline 0 & -1 & 1 & $-\sqrt{3 / 20 \pi}$ & $T_{1}$ \\
\hline 0 & 1 & -1 & $-\sqrt{3 / 20 \pi}$ & $T_{-1}$ \\
\hline
\end{tabular}


In Table 3.2 we compile the results for the orbital integrals for $\ell^{\prime}=\ell=1$ already including the factors to change the middle harmonic $Y_{2}^{q}(\theta, \varphi)$ into the specific operator $T$ from eqs. (3.41), (3.42) and (3.43), and also the factor 4 in the previous definition of the tensor operator in eq. (3.6) and later in eq. (3.40). Then the only factors missing from the following results are the Clebsh-Gordan coefficients and the spin part, both which will differ in each expectation value we take of the different wavefunctions.

Table 3.2: Results for the orbital integrals with $\ell^{\prime}=\ell=1$ including the factors of the $T$ operators and the factor 4 for notation consistence, without the spin part or Clebsh-Gordan coefficients.

\begin{tabular}{|c|c|c|c|c|}
\hline $\mathrm{m}$ & $\mathrm{q}$ & $\mathrm{m}$ & Operator & $4 \int Y_{\ell^{\prime}}^{m^{\prime} *}(\theta, \varphi) T_{\ldots}(\theta, \varphi) Y_{\ell}^{m}(\theta, \varphi) \mathrm{d} \Omega$ \\
\hline 1 & 0 & 1 & $T_{0}$ & $-8 / 5$ \\
\hline 1 & 0 & 1 & $T_{0}^{\prime}$ & $2 / 5$ \\
\hline-1 & 0 & -1 & $T_{0}$ & $-8 / 5$ \\
\hline-1 & 0 & -1 & $T_{0}^{\prime}$ & $2 / 5$ \\
\hline 0 & 0 & 0 & $T_{0}$ & $16 / 5$ \\
\hline 0 & 0 & 0 & $T_{0}^{\prime}$ & $-4 / 5$ \\
\hline 1 & 2 & -1 & $T_{-2}$ & $-12 / 5$ \\
\hline-1 & -2 & 1 & $T_{2}$ & $-6 \sqrt{2} / 5$ \\
\hline 1 & 1 & 0 & $T_{-1}$ & $6 \sqrt{2} / 5$ \\
\hline-1 & -1 & 0 & $T_{1}$ & $-6 \sqrt{2} / 5$ \\
\hline 0 & -1 & 1 & $T_{1}$ & $6 \sqrt{2} / 5$ \\
\hline 0 & 1 & -1 & $T_{-1}$ & \\
\hline
\end{tabular}


Finally, in Table 3.3 we compile the results for the tensor factor (using the results from the previous Tables 3.1 and 3.2) calculated considering both diquark and antidiquark with total spin 1, (which can couple into the tetraquark total spin $S_{T}=0,1,2$ ), and with relative orbital momentum in the diquark-antidiquark system $L_{T}=1$ (no orbital momentum inside diquark or antidiquark). In this case there are seven possibilities of total angular momentum $J_{T}$ in the tetraquark. The tensor factor is calculated considering only the diagonal matrix element: $\left\langle\mathbf{S}_{\mathbf{d}-\overline{\mathbf{d}}}\right\rangle=\left\langle S_{T}, L_{T}, J_{T}\left|\mathbf{S}_{\mathbf{d}-\overline{\mathbf{d}}}\right| S_{T}, L_{T}, J_{T}\right\rangle$ in the principal case of the $J_{T}$ Z-component $M_{J_{T}}=J_{T}$.

Table 3.3: Results for the tensor factor with $\left[S_{d}=S_{\bar{d}}=1 \rightarrow S_{T}=0,1,2\right] \otimes$ $L_{T}=1$.

\begin{tabular}{|c|c|c|c|}
\hline$S_{T}$ & $L_{T}$ & $J_{T}$ & $\left\langle\mathbf{S}_{\mathbf{d}-\overline{\mathbf{d}}}\right\rangle$ \\
\hline 0 & 1 & 1 & 0 \\
\hline 1 & 1 & 2 & $-\frac{4}{5}=-0,8$ \\
\hline 1 & 1 & 1 & 4 \\
\hline 1 & 1 & 0 & -8 \\
\hline 2 & 1 & 3 & $-\frac{8}{5}=-1,6$ \\
\hline 2 & 1 & 2 & $\frac{28}{5}=5,6$ \\
\hline 2 & 1 & 1 & $-\frac{561}{100}=-5,61$ \\
\hline
\end{tabular}

Notice the results have a similar structure to the ones for two spin $1 / 2$ particles. There we had that the tensor factor is zero for total spin 0 , here we have the same as well. There when the total spin was 1 and the orbital momentum also $\ell=1$, we had a small negative number for $J=\ell+1=2$, a positive number for $J=\ell=1$ and a big negative number for $J=\ell-1=0$. Interestingly, for $S_{T}=1$ and $L_{T}=1$ in the tetraquark (with spin 1 diquark and antidiquark), we have, with an extra factor 2, exactly the same numbers of the case of two spin $1 / 2$ and $\ell=1\left(-\frac{2}{5},+2,-4\right)$ as we commented after eq. (3.35). And here, with $S_{T}=2$ we still have a similar structure of positive and negative numbers depending on the relation of $J_{T}$ and $L_{T}$. Probably, with more insight in the algebraic manipulation of the operators, one could obtain a similar general result for the expectation value of the tensor operator in the coupling of two spin 1 particles, or even in a completely general case for any values of spin. 
Now there is a very important observation to be made. The fact that both approaches (either with four tensors applied on a wavefunction with four spins $1 / 2$, or one tensor applied on a wavefunction with two spins 1) lead to the same result is not a coincidence. Actually, it should have been obvious from the beginning, because what we did was just change the spin basis from $\left\{\frac{1}{2} \otimes \frac{1}{2} \otimes \frac{1}{2} \otimes \frac{1}{2}\right\}$ to $\{1 \otimes 1\}$, where the Clebsh-Gordan coefficients play an important role. Of course, thinking about the physics of the model, this equivalence only was possible because we used the approximation that the distances between every $q-\bar{q}$ pair are exactly the same in the diquark-antidiquark system, since we treat the later as a 2-body problem $i$. e., the interaction of two point-like structures. In a real 4-body approach (using Jacobi coordinates for example), where all the four constituents are allowed to move "freely" and interact at the same time with each other, this would not be true. This type of approach can be found in other models of tetraquarks, for instance [73, 74], but sometimes in this kind of model only the ground state is considered, with no orbital excitations, then only the spin-spin interaction is relevant since the spin-orbit and tensor vanish for $\ell=0$.

\subsection{The Spin-Average}

The spin-dependent interactions are an important feature of charmonium spectroscopy because they serve as a testing scenario of the QCD dynamics in heavy quark context, lying between perturbative and non-perturbative regimes. One particularly interesting subject is the role of the spin-spin interaction in orbitally excited states. It is convenient to define the spin-average (spin here means $J$ ) of a multiplet, also know as "center-of-weight" or "centerof-gravity" (c.o.g.):

$$
\left.\left\langle M\left(N^{2 S+1} \ell_{J}\right)\right\rangle=\frac{\sum_{J}(2 J+1) M\left(N^{2 S+1} \ell_{J}\right)}{\sum_{J}(2 J+1)}, \quad \text { (c.o.g. }\right)
$$

For P-wave ground state for example, we have:

$$
\left\langle M\left(1^{3} P_{J}\right)\right\rangle=\frac{5 M\left(1^{3} P_{2}\right)+3 M\left(1^{3} P_{1}\right)+M\left(1^{3} P_{0}\right)}{9}
$$

The interesting point of the spin-average mass is that the spin-orbit and tensor corrections cancel themselves, so if the spin-spin correction is zero in the orbitally excited singlet state, its mass should be equal to this spin average. The experimental measurement of the P-wave ground states of charmonium seem to agree with that (see Chapter 4).

It is simple to verify that the spin corrections vanish for the c.o.g. As presented in Ref. [63], if we put together the spin-orbit and tensor terms, we can write their contribution as:

$$
\left\langle V_{L S}^{(1)}\right\rangle+\left\langle V_{T}^{(1)}\right\rangle=\frac{1}{m^{2}}\left[A_{J} \alpha_{s}\left\langle\frac{1}{r^{3}}\right\rangle+B_{J} b\left\langle\frac{1}{r}\right\rangle\right]
$$

For two spin $1 / 2$ particles in $\mathrm{P}$-wave meson, using the results in eqs. (3.15), (3.19), (3.21) and (3.35), we have the following coefficients: 
Table 3.4: $A_{J}$ and $B_{J}$ coefficients for P-wave mesons.

\begin{tabular}{ccc}
\hline State & $A_{J}$ & $B_{J}$ \\
\hline${ }^{3} P_{2}$ & $-(28 / 15)$ & $-(1 / 2)$ \\
${ }^{3} P_{1}$ & $-(4 / 3)$ & $+(1 / 2)$ \\
${ }^{3} P_{0}$ & $-(16 / 3)$ & +1 \\
${ }^{1} P_{1}$ & 0 & 0 \\
\hline
\end{tabular}

Notice that both terms vanish exactly for the c.o.g. average of the P-wave:

$$
\begin{aligned}
& A_{J} \rightarrow[5(28 / 15)+3(-4 / 3)+(-16 / 3)] / 9=0 \\
& B_{J} \rightarrow[5(-1 / 2)+3(+1 / 2)+(1)] / 9=0
\end{aligned}
$$

Now remember that in our first model the mass of the spin singlet orbitally excited states is the $M^{(0)}$ mass, since the three spin-corrections are zero. Then its mass is exactly equal to the mass of the center-of-gravity. For the second model, since we include the spin-spin interaction non-perturbatively, it actually has a small but non-negligible contribution, and it also causes a small difference in the wavefunctions of spin singlets and spin triplets. Therefore the c.o.g. mass is not equal to the spin singlet mass. This is a very interesting peculiarity of this second model, which we are going to further discuss in Chapter 4 with the theoretical results for charmonium and its comparisons with experimental data. 


\section{Chapter 4}

\section{Mesons}

In order to make good estimates of our diquarks and tetraquarks, we first study the spectrum of charmonium mesons, to observe how well we can fit the experimental data with both models.

Seeking the best set of parameters, we employ a fitting procedure minimizing the $\chi^{2}$ with Mathematica, considered as the sum of the square of the differences between the mass calculated with our model and the (experimentally measured) candidate for that particular choice of quantum numbers, multiplied by its statistical weight (for $n$ states used as input):

$$
\chi^{2}=\sum_{i}^{n}\left(M_{i}^{\text {calc }}-M_{i}^{\text {exp }}\right)^{2} \cdot w_{i}
$$

As discussed in Ref. [62], using the experimental error as statistical weight $w_{i}=\left(\sigma_{i}^{2}\right)^{-1}$ strongly bias the results to the states that have very small experimental error, like $J / \psi$, and doesn't force the parameters to fit the other states. If we use the error and the decay width $w_{i}=\left(\sigma_{i}^{2}+\left(\frac{\Gamma_{i}}{2}\right)^{2}\right)^{-1}$ we still wouldn't force the method to fit the broad resonances, like $\eta_{c}$ and the ones above the $D \bar{D}$ threshold (that can decay in open charm meson pairs). Since we wish to obtain an overall fit, that would allow reasonable predictions to missing $c \bar{c}$ states, we choose $w_{i}=1$, which is equivalent to give the same statistical weight to all the states used as input, the same strategy adopted in Refs. $[62,67]$ and many others spectroscopy studies. (Actually, in Ref. [62] they also perform fits using for instance only the 8 states below the threshold, but as we will see with the second model of Refs. [66, 67], it is possible to obtain good agreement with all the experimental data with these potential models).

In the following sections we will discuss the trajectory we went through in the search for a satisfactory fit of the charmonium spectrum and the reasons we had to adopt the second model but still not completely abandon the first one.

We use the following notation in our result tables: the principal quantum number is $N(N=1$ for the ground state, $N=2$ for the first radial excitation and so on), $\ell$ is the orbital angular momentum, $S$ is the total spin and $J$ the total angular momentum. In spectroscopy notation the states are usually labeled by $N^{2 S+1} \ell_{J}$, with $\ell=0,1,2,3,4, \ldots \rightarrow$ $S, P, D, F, G, \ldots$, for example $1^{3} S_{1}$ for $J / \psi$.

It is common to characterize the bound states by the $J^{P C}$ quantum numbers, where $J$ is the total angular momentum, which is the one that is actually conserved from the relativistic point-of-view (remember we also consider $S$ and $\ell$ as good quantum number). While $P$ and $C$ are the parity and charge-conjugation quantum numbers, respectively, which are related 
to symmetries.

In simple words, the parity operator produces a reflection in the coordinates relative to the origin of the system (like a mirror). The parity of the spherical harmonics brings a factor $(-1)^{\ell}$, where $\ell$ is the orbital quantum number. An additional $(-1)$ factor is included to account for the particle-antiparticle structure, so we have $P=(-1)^{\ell+1}$.

The charge-conjugation operator changes a particle by its antiparticle. It can be shown that for mesons the charge-conjugation quantum number is given by $C=(-1)^{\ell+S}$, where $S$ is the total spin (further discussion can be found in Chapter 4 of Ref. [1], for instance). Of course not every bound state of quarks is an eigenstate of both operators, but $Q \bar{Q}$ mesons are (with eigenvalues +1 or -1 ).

$$
\begin{aligned}
& P=(-1)^{\ell+1} \\
& C=(-1)^{\ell+S}
\end{aligned}
$$

Other operators related to symmetries are isospin (from light quark symmetry) and Gparity (that is a generalization of parity, charge-conjugation and isospin), but since in this work we treat only states composed of charm quarks, isospin and g-parity are not relevant in our discussion.

The experimental data are presented as in the PDG of the 2014 edition with important updates from 2015 [52].We adopt as well-established the $13 c \bar{c}$ states indicated in Table 4.8. The input values used in the fitting procedures are also used with as many digits as provided by the PDG. The comparisons of the results using model 1 and model 2 with experimental data are presented in Section 4.3.

We point that once the parameters of a particular fit are obtained, they are kept fixed to generate the whole spectrum. Effects like the decreasing of the strong coupling constant with increase of energy, relativistic momentum-dependent contributions or others related to the open charm mesons aren't taken into consideration.

Several attempts to obtain reasonable agreement of the calculated values with the whole experimental spectrum of charmonium were made with different inputs and assumptions. In Table 4.7 of Section 4.3 we compile the results for the potential parameters obtained in each fit.

\subsection{Charmonium: Model 1}

In the first model we consider the zeroth-order potential of the form Coulomb plus linear. A charmonium meson is a quark-antiquark system $(c \bar{c})$ bound in a color singlet configuration. As shown in Section 2.4, the color factor for one gluon exchange between quark and antiquark bound in the color singlet configuration is $\kappa_{s}=-\frac{4}{3}$. So the potential illustrated in Fig. 4.1 that we put in the Schrödinger equation is:

$$
V_{1}^{(0)}(r)=-\frac{4}{3} \frac{\alpha_{s}}{r}+b r
$$




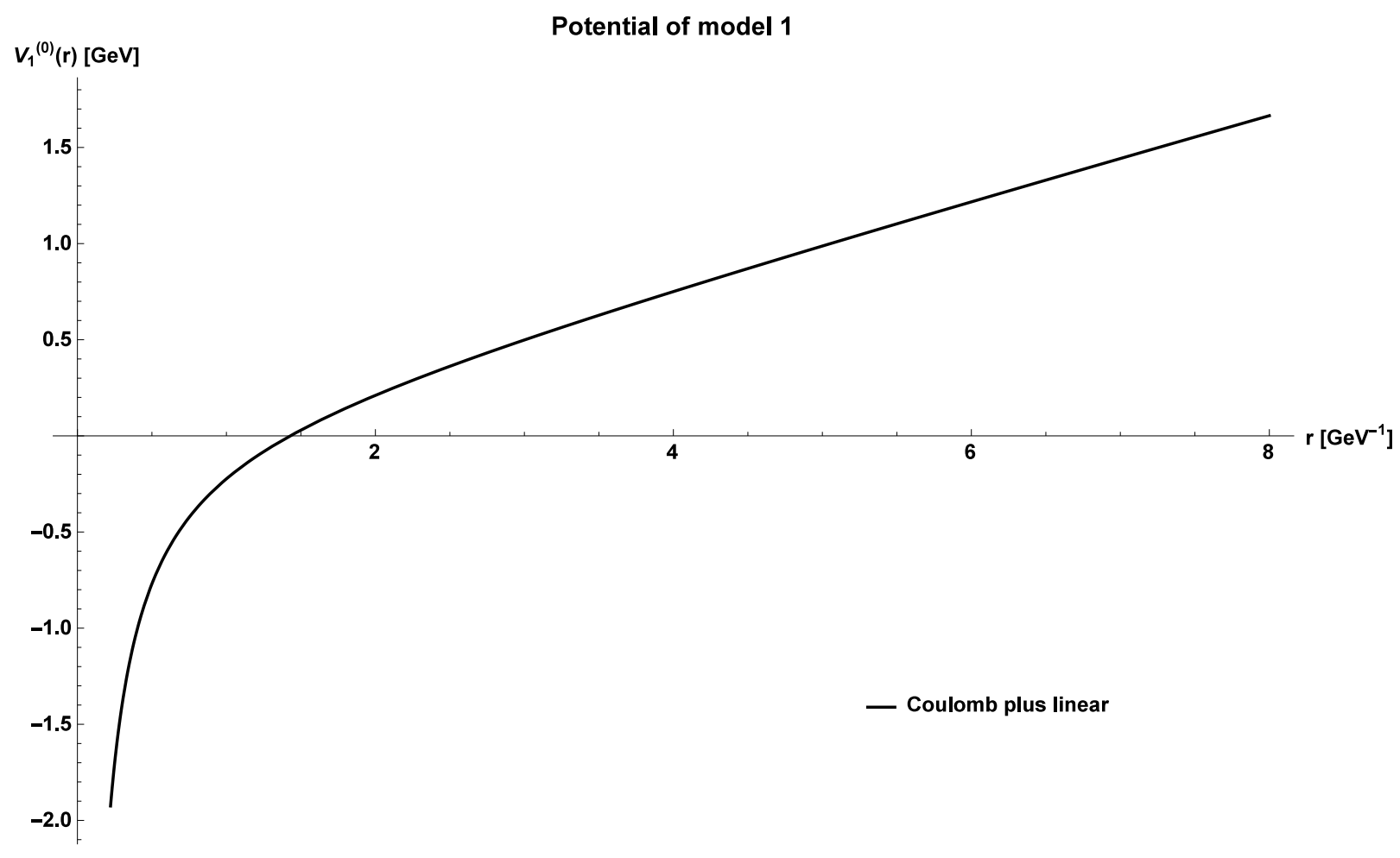

Figure 4.1: Potential of model 1: Coulomb plus linear. Parameters are $\alpha_{s}=$ $0.3289, b=0.2150 \mathrm{GeV}^{2}$. is:

The spin-dependent potential (first-order perturbation) between the spin $1 / 2$ constituents

$$
\left\langle V_{1, \text { Spin }}^{(1)}(r)\right\rangle=\frac{1}{m^{2}}\left[\frac{8 \alpha_{s}}{9}|R(0)|^{2}\left\langle\mathbf{S}_{\mathbf{1}} \cdot \mathbf{S}_{\mathbf{2}}\right\rangle+\left(2 \alpha_{s}\left\langle\frac{1}{r^{3}}\right\rangle-\frac{b}{2}\left\langle\frac{1}{r}\right\rangle\right)\langle\mathbf{L} \cdot \mathbf{S}\rangle+\frac{\alpha_{s}}{3}\left\langle\frac{1}{r^{3}}\right\rangle\left\langle\mathbf{S}_{\mathbf{1 2}}\right\rangle\right]
$$

\subsubsection{Model 1: Fit with 13 input}

First we present the result of the fit performed with all the $13 c \bar{c}$ states as input and the choice of quantum numbers of each one as it is commonly adopted in the literature (in Refs. $[62,67]$ for example). See Table 4.8 in section 4.3 for the details about the experimental data.

We considered as free parameters in the fitting procedure the mass of the charm quark $m_{c}$, the strong coupling constant $\alpha_{s}$ and the string tension $b$, allowing them to vary within the following range:

$$
1.1<m_{c}<1.9 \mathrm{GeV}, \quad 0.1<\alpha_{s}<0.7, \quad 0.050<b<0.450 \mathrm{GeV}^{2}
$$

The parameters and $\chi^{2}$ obtained were:

$$
\chi^{2}=0.0132 \mathrm{GeV}^{2}, \quad m_{c}=1.4155 \mathrm{GeV}, \quad \alpha_{s}=0.4116, \quad b=0.1517 \mathrm{GeV}^{2}
$$

A rough estimate of the theoretical error in the fitting procedure could be calculated as:

$$
\text { error } \approx \frac{\sqrt{\chi^{2}}}{n}, \quad(\mathrm{n}=\text { number of input states })
$$


In this case we have $n=13$, resulting in an estimated error of $8.8 \mathrm{MeV}$ (per input).

The plot of the calculated values (dashed line on the right) and experimental data (continuous line on the left) are shown in Figure 4.2. We have calculated the complete multiplet of each of the charmonium experimentally known states, even tough not every multiplet is completely measured (see table 4.8 for details of the experimental data).

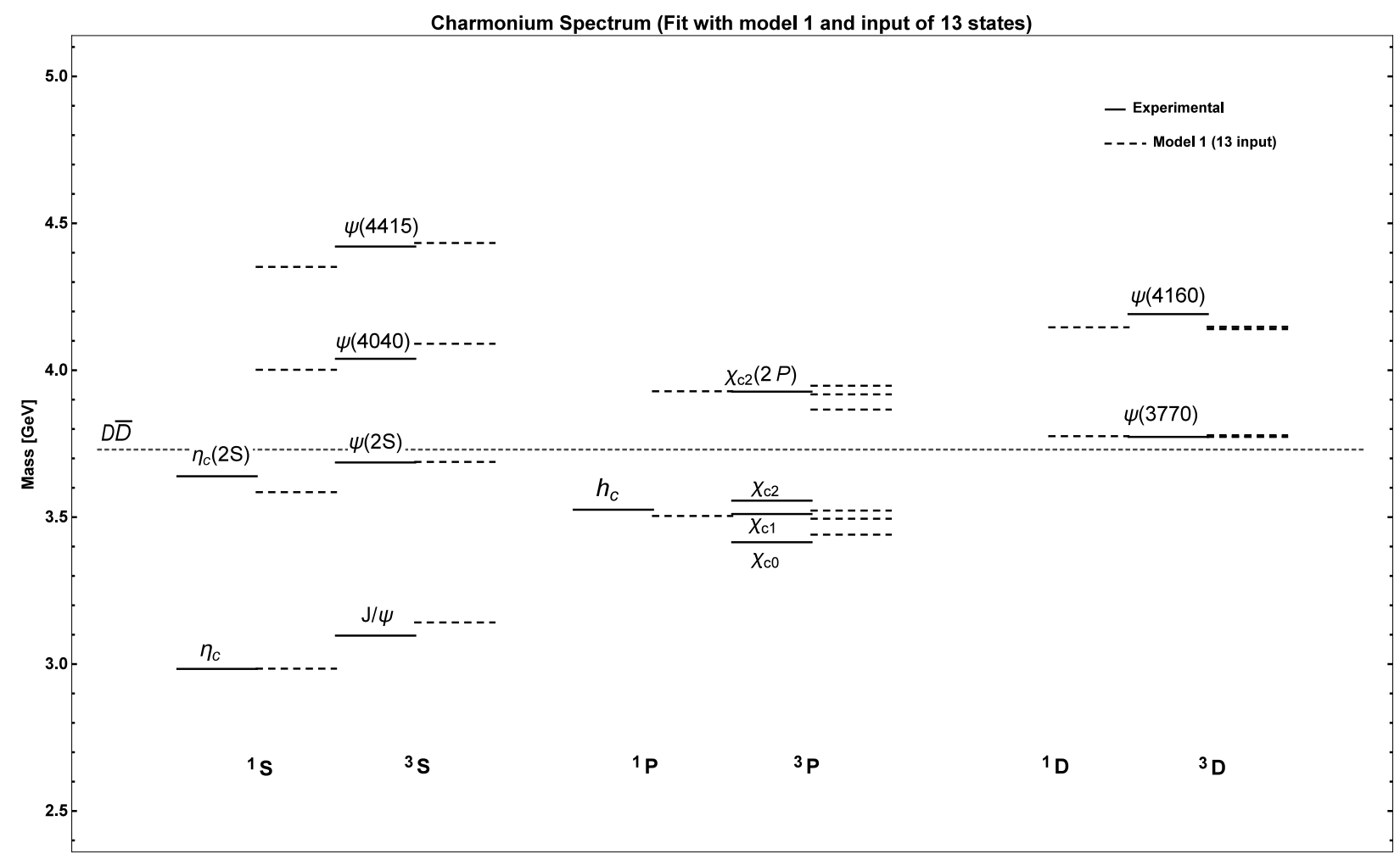

Figure 4.2: Spectrum of charmonium obtained with model 1 and input of 13 $c \bar{c}$ states. Parameters are $m_{c}=1.4155 \mathrm{GeV}, \alpha_{s}=0.4116$ and $b=0.1517$ $G e V^{2}$.

We show in the spectrum the first $D \bar{D}$ threshold at $3.73 \mathrm{GeV}$. There are other thresholds above this one (see [52]), corresponding to heavier open charm meson pairs. We could use that to imagine how confinement works. One could picture it as a string binding quark and antiquark. The potential energy increases as the distance between them increases (due to radial and/or orbital excitations), and so does the mass of the state, until the point when there is enough energy to create a new quark-antiquark pair, allowing the $c \bar{c}$ to decay into a charmed meson pair, like $D \bar{D}(c \bar{u}+u \bar{c})$. This is possible only for states above the threshold.

In all tables the results presented in $\mathrm{GeV}$ (like $M^{(0)}$, the zeroth-order mass) are rounded up to the fourth decimal digit, while the ones presented in $\mathrm{MeV}$ (like $M^{f}$, the final value for the mass where we include the perturbative corrections) are rounded up to the first decimal digit. A few of the rounded values in the tables might not perfectly sum up to the final mass due to the fractions after the last digit that are used in the calculations.

In Table 4.1 we present the zeroth-order results (in order of increasing experimental mass, approximately) for the wavefunctions obtained solving the Schrödinger equation with the Coulomb plus linear potential. Notice that the wavefuntions depend only on the principal quantum number $N$ and orbital $\ell$ since all spin-dependent terms are treated perturbatively. 


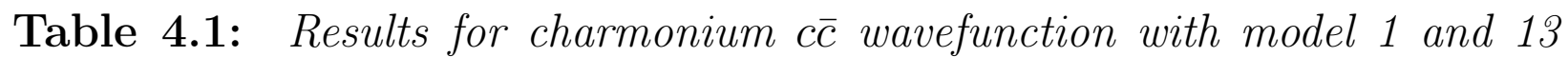
input states. Parameters are $m_{c}=1.4155 \mathrm{GeV}, \alpha_{s}=0.4116$ and $b=0.1517$ $G e V^{2}$.

\begin{tabular}{crrrr}
\hline \hline$N \ell$ & $M^{(0)}[\mathrm{GeV}]$ & $|R(0)|^{2}\left[\mathrm{GeV}^{3}\right]$ & $\left\langle r^{2}\right\rangle^{1 / 2}[\mathrm{fm}]$ & $\left\langle\frac{v^{2}}{c^{2}}\right\rangle$ \\
\hline $1 S$ & 3.1024 & 0.8608 & 0.444 & 0.240 \\
\hline $1 P$ (c.o.g.) & 3.5041 & 0 & 0.708 & 0.248 \\
\hline $2 S$ & 3.6623 & 0.5638 & 0.889 & 0.298 \\
\hline $1 D$ (c.o.g.) & 3.7756 & 0 & 0.916 & 0.287 \\
\hline $2 P$ (c.o.g.) & 4.1460 & 0 & 1.274 & 0.363 \\
\hline $3 S$ & 4.0680 & 0.4848 & 1.248 & 0.370 \\
\hline $2 D$ (c.o.g.) & 4.1460 & 0 & 1.274 & 0.363 \\
\hline $4 S$ & 4.4127 & 0.4453 & 1.563 & 0.439 \\
\hline \hline
\end{tabular}

The mass value before spin corrections is $M^{(0)}$, composed only of the mass of the charm quarks plus the Schrödinger equation eigenenergy. The square of the radial wavefunction at the origin is $|R(0)|^{2}$, the root mean square radius is $\left\langle r^{2}\right\rangle^{1 / 2}$ (in fermi) and the quark mean square velocity in units of $c^{2}$ is $\left\langle\frac{v^{2}}{c^{2}}\right\rangle$.

Next, we include the spin-dependent interactions as corrections in the total mass, treated as first-order perturbation using the wavefunctions previously calculated.

In Table 4.2 we present the details of the calculation of each particular state: the contribution of each perturbative correction and also the expectation value of the kinetic energy, the vector (Coulomb) and scalar (linear) terms of the zeroth-order potential, in order to observe how much each of them contribute to the energy eigenvalue, what gives us an idea of which QCD regime is dominant in each state, the one gluon exchange (perturbative QCD) or the confinement regime. The pattern is clear, the kinetic energy and confinement contributions increase with radial and orbital excitations, while the vector contribution decreases. 
Table 4.2: Results for charmonium cō masses (including spin-dependent corrections) with model 1 and 13 input states. Parameters are $m_{c}=1.4155$ $G e V, \alpha_{s}=0.4116$ and $b=0.1517 G e V^{2}$.

\begin{tabular}{crrrrrrrrr}
\hline \hline$N^{2 S+1} \ell_{J}$ & $\langle T\rangle$ & $\left\langle V_{V}^{(0)}\right\rangle$ & $\left\langle V_{S}^{(0)}\right\rangle$ & $E^{(0)}$ & $M^{(0)}[\mathrm{MeV}]$ & $\left\langle V_{S S}^{(1)}\right\rangle$ & $\left\langle V_{L S}^{(1)}\right\rangle$ & $\left\langle V_{T}^{(1)}\right\rangle$ & $M_{1}^{f}[\mathrm{MeV}]$ \\
\hline $1^{1} S_{0}$ & 340.3 & -374.7 & 305.9 & 271.4 & 3102.4 & -118.0 & 0 & 0 & 2984.5 \\
$1^{3} S_{1}$ & 340.3 & -374.7 & 305.9 & 271.4 & 3102.4 & 39.3 & 0 & 0 & 3141.7 \\
\hline $1^{3} P_{0}$ & 350.6 & -189.3 & 511.9 & 673.1 & 3504.1 & 0 & -41.2 & -22.4 & 3440.4 \\
$1^{3} P_{1}$ & 350.6 & -189.3 & 511.9 & 673.1 & 3504.1 & 0 & -20.6 & 11.2 & 3494.7 \\
$1^{1} P_{1}$ (c.o.g.) & 350.6 & -189.3 & 511.9 & 673.1 & 3504.1 & 0 & 0 & 0 & 3504.1 \\
$1^{3} P_{2}$ & 350.6 & -189.3 & 511.9 & 673.1 & 3504.1 & 0 & 20.6 & -2.2 & 3522.4 \\
\hline $2^{1} S_{0}$ & 421.9 & -217.2 & 626.7 & 831.3 & 3662.3 & -77.2 & 0 & 0 & 3585.1 \\
$2^{3} S_{1}$ & 421.9 & -217.2 & 626.7 & 831.3 & 3662.3 & 25.7 & 0 & 0 & 3688.0 \\
\hline $1^{3} D_{1}$ & 405.7 & -136.2 & 675.1 & 944.6 & 3775.6 & 0 & 0.6 & -3.1 & 3773.1 \\
$1^{3} D_{2}$ & 405.7 & -136.2 & 675.1 & 944.6 & 3775.6 & 0 & 0.2 & 3.1 & 3778.8 \\
$1^{1} D_{2}$ (c.o.g.) & 405.7 & -136.2 & 675.1 & 944.6 & 3775.6 & 0 & 0 & 0 & 3775.6 \\
$1^{3} D_{3}$ & 405.7 & -136.2 & 675.1 & 944.6 & 3775.6 & 0 & -0.4 & -0.9 & 3774.3 \\
\hline $2^{3} P_{0}$ & 462.6 & -145.2 & 780.0 & 1097.5 & 3928.4 & 0 & -41.7 & -20.6 & 3866.1 \\
$2^{3} P_{1}$ & 462.6 & -145.2 & 780.0 & 1097.5 & 3928.4 & 0 & -20.9 & 10.3 & 3917.9 \\
$2^{1} P_{1}$ (c.o.g.) & 462.6 & -145.2 & 780.0 & 1097.5 & 3928.4 & 0 & 0 & 0 & 3928.4 \\
$2^{3} P_{2}$ & 462.6 & -145.2 & 780.0 & 1097.5 & 3928.4 & 0 & 20.9 & -2.1 & 3947.2 \\
\hline $3^{1} S_{0}$ & 524.1 & -167.7 & 880.6 & 1237.0 & 4068.0 & -66.4 & 0 & 0 & 4001.6 \\
$3^{3} S_{1}$ & 524.1 & -167.7 & 880.6 & 1237.0 & 4068.0 & 22.1 & 0 & 0 & 4090.1 \\
\hline $2^{3} D_{1}$ & 514.3 & -113.9 & 914.7 & 1315.1 & 4146.0 & 0 & -3.1 & -3.0 & 4140.0 \\
$2^{3} D_{2}$ & 514.3 & -113.9 & 914.7 & 1315.1 & 4146.0 & 0 & -1.0 & 3.0 & 4148.0 \\
$2^{1} D_{2}$ (c.o.g.) & 514.3 & -113.9 & 914.7 & 1315.1 & 4146.0 & 0 & 0 & 0 & 4146.0 \\
$2^{3} D_{3}$ & 514.3 & -113.9 & 914.7 & 1315.1 & 4146.0 & 0 & 2.1 & -0.8 & 4147.2 \\
\hline $4^{1} S_{0}$ & 621.4 & -141.3 & 1102.0 & 1581.8 & 4412.7 & -61.0 & 0 & 0 & 4351.7 \\
$4^{3} S_{1}$ & 621.4 & -141.3 & 1102.0 & 1581.8 & 4412.7 & 20.3 & 0 & 0 & 4433.0 \\
\hline \hline
\end{tabular}

Notice that the spin-spin interaction splits singlet and triplet only in S-wave states, because it is proportional to the square of the wavefunction at the origin, which is zero in the orbitally excited states due to the centrifugal barrier (as discussed in Section 2.6).

The spin-orbit and tensor appear only in spin triplets with non-zero orbital momentum and they split the orbitally excited states into four states, three are spin triplets with different values of total angular momentum $J$ and one is the spin singlet, where all three spin corrections vanish, so its mass is simply $M^{(0)}$.

As discussed in Section 3.4, the mass of the spin-average (remember spin-orbit and tensor cancel when the average is taken) or "center-of-gravity" of an orbitally excited spin triplet (total spin 1) is equal to mass of the corresponding spin singlet (total spin 0), which has all 
three-spin corrections vanishing. That happens in model 1 because the spin-spin correction is zero for any orbitally excited state, so only spin-orbit and tensor play a role in splitting. There is an experimental example for that: the $1 P$ multiplet (see Table 4.8). The mass of the c.o.g. of the spin 1 states $\chi_{c 0}\left(1^{3} P_{0}\right), \chi_{c 1}\left(1^{3} P_{1}\right)$ and $\chi_{c 2}\left(1^{3} P_{2}\right)$ is compatible with the mass of the spin 0 state $h_{c}\left(1^{1} P_{1}\right)$.

\subsubsection{Model 1 with constant $V_{0}$ : Fit with 13 input}

Hereafter we will present a series of additional efforts to fit the model into the experimental data. Some extra tables and figures can be found in the appendix A.

Trying to improve the agreement of the calculated values with the experimental input, we included a constant term $V_{0}$ in the zeroth-order potential, which can be justified as a point-zero energy with partially scalar and/or vector origin (see Refs. [58, 62] for example). We used the same input of 13 states and same range for the last three parameters, including the constant term as a fourth free parameter, allowed to vary within the wide range $-2<$ $V_{0}<2 \mathrm{GeV}$, resulting in the following set:

$\chi^{2}=0.0124 \mathrm{GeV}^{2}, \quad m_{c}=1.7378 \mathrm{GeV}, \quad \alpha_{s}=0.4066, \quad b=0.15945 \mathrm{GeV}^{2}, \quad V_{0}=-0.5871 \mathrm{GeV}$

The estimated error now is about $8.6 \mathrm{MeV}$ (per input). It turns out that the result is practically the same: the constant term shifts the whole spectrum and causes the charm quark mass to increase (the $\alpha_{s}$ and the string tension $b$ are practically unchanged). The resulting parameters produce the same pattern in the mass spectrum as before, and the $\chi^{2}$ is just a little smaller than the previous one.

\subsubsection{Model 1: Fit with 6 input (1S and 1P)}

Next, trying to improve the orbital splitting of the P-wave mesons, we use as input only the 6 states with $N=1$ below the $D \bar{D}$ threshold: $\eta_{c}\left(1^{1} S_{1}\right), J / \psi\left(1^{3} S_{1}\right), h_{c}\left(1^{1} P_{1}\right), \chi_{c 0}\left(1^{3} P_{0}\right)$, $\chi_{c 1}\left(1^{3} P_{1}\right)$ and $\chi_{c 2}\left(1^{3} P_{2}\right)$. The parameters were allowed to vary in the following range:

$$
1.1<m_{c}<1.9 \mathrm{GeV}, \quad 0.1<\alpha_{s}<0.6, \quad 0.050<b<0.400 \mathrm{GeV}^{2}
$$

Resulting in:

$$
\chi^{2}=0.0028 \mathrm{GeV}^{2}, \quad m_{c}=1.3465 \mathrm{GeV}, \quad \alpha_{s}=0.3796, \quad b=0.1857 \mathrm{GeV}^{2}
$$

The values of the parameters changed a little: $m_{c}$ and $\alpha_{s}$ decreased, while $b$ increased. The agreement in the spin-spin splitting of the $1 S$ states got better, but the $1 P$ orbital splitting was still smaller than expected. The $\chi^{2}$ is smaller this time, but the estimated error has to be divided by 6 input states, and it only estimates the error for these 6 states, not for the rest of the spectrum. The result is $8.9 \mathrm{MeV}$ (per input), similar to the previous ones, what indicates that reducing to only this 6 states, where both the singlet-triplet and the orbital splitting are tested simultaneously, doesn't make it easier to accommodate the model into the data. 


\subsubsection{Model 1 with modified Spin-Orbit: Fit with 6 input (1S and 1P)}

Observing the results of similar quarkonium models (as for example the Breit interaction for one gluon exchange of Ref. [65]) we thought that the cause of that shrinking in the Pwave splitting could be the second term of the spin-orbit interaction, which is proportional to the string tension $b$ (and it is not present in the cited reference). This term comes from the assumption that confinement is due to a Lorentz scalar, and according to $[59,60,66,67]$ it is a Thomas precession effect.

Then we performed the same fit using the $1 S$ and $1 P$ states as input, but now removing the second term of the spin-orbit interaction. The result was a much better agreement of these 6 states, even though the rest of the spectrum didn't match well, specially the radial excitations. The result for the parameters are:

$$
\chi^{2}=0.0004 \mathrm{GeV}^{2}, \quad m_{c}=1.2819 \mathrm{GeV}, \quad \alpha_{s}=0.3289, \quad b=0.2150 \mathrm{GeV}^{2}
$$

The $\chi^{2}$ is much smaller this time, dividing by 6 input states we got an estimated error of $3.1 \mathrm{MeV}$ (for the 6 states only). It is interesting to notice that the charm quark mass is very close to value given in the PDG summary [52] (with update 2015): $m_{c}=1.275 \pm 0.025 \mathrm{GeV}$ ("running" mass in the $\overline{M S}$ scheme). The value of the strong coupling constant $\alpha_{s}$ around 0.3 is also more typical in potential models of charmonium. In Figure 4.3 we plot the mass spectrum with this set.

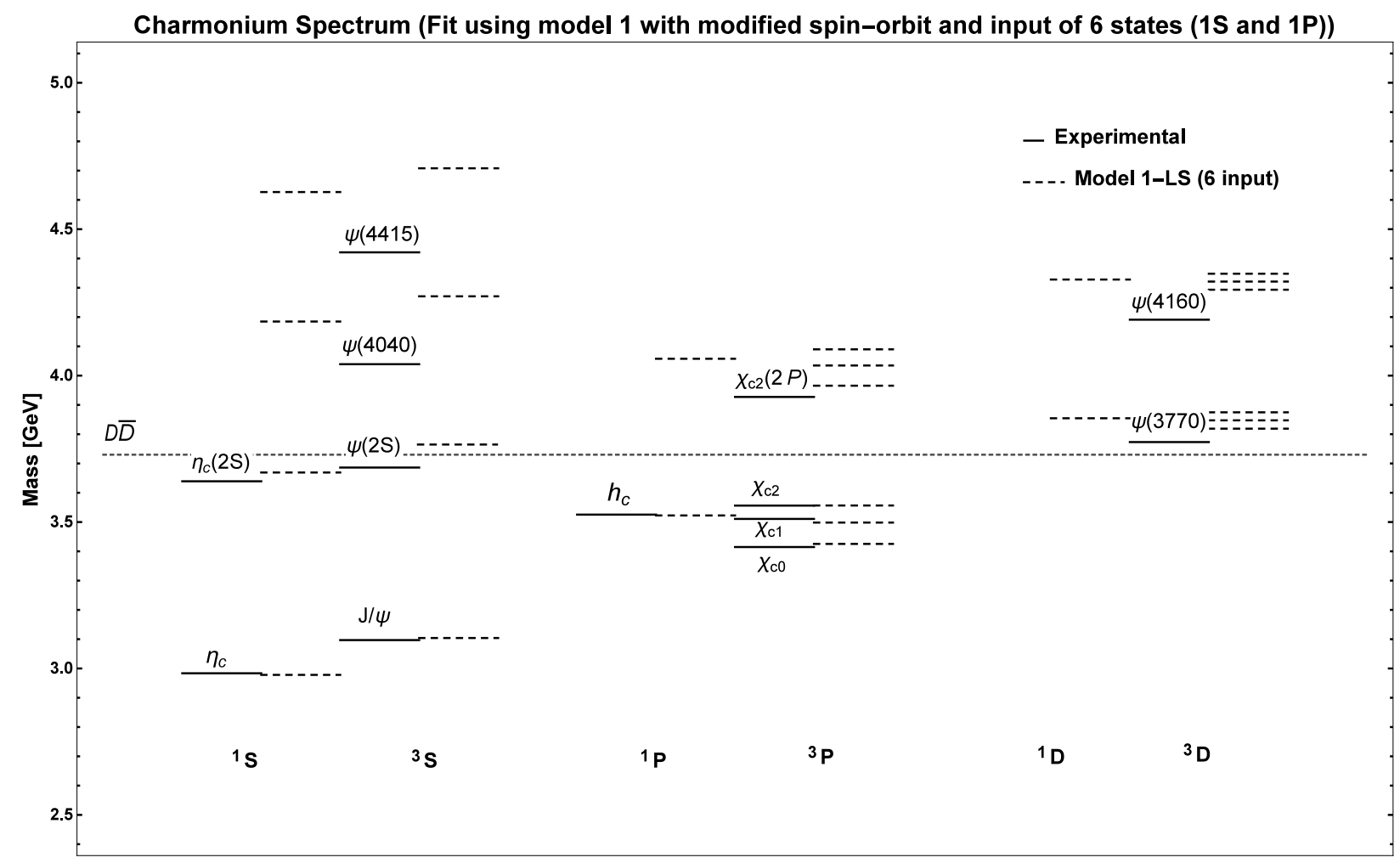

Figure 4.3: Spectrum of charmonium with model 1, input of 6 c $\bar{c}$ states $(1 S$ and $1 P$ ) and modified spin-orbit interaction. Parameters are $m_{c}=1.2819$ GeV, $\alpha_{s}=0.3289, b=0.2150 \mathrm{GeV}^{2}$. 
The results for wavefunctions and masses with this set are in Tables 4.3 and 4.4:

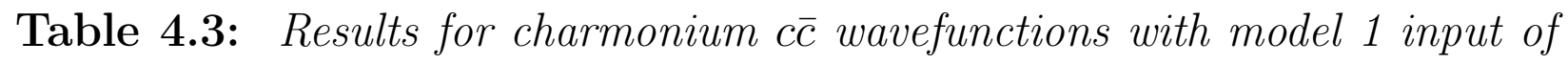
$6 c \bar{c}$ states $(1 S$ and $1 P)$ and modified spin-orbit interaction. Parameters are $m_{c}=1.2819, \mathrm{GeV}, \alpha_{s}=0.3289, b=0.2150 \mathrm{GeV}^{2}$.

\begin{tabular}{crrrr}
\hline \hline$N \ell$ & $M^{(0)}[\mathrm{GeV}]$ & $|R(0)|^{2}\left[\mathrm{GeV}^{3}\right]$ & $\left\langle r^{2}\right\rangle^{1 / 2}[\mathrm{fm}]$ & $\left\langle\frac{v^{2}}{c^{2}}\right\rangle$ \\
\hline $1 S$ & 3.0725 & 0.7075 & 0.444 & 0.284 \\
\hline $1 P$ (c.o.g.) & 3.5225 & 0 & 0.674 & 0.331 \\
\hline $2 S$ & 3.7408 & 0.5368 & 0.847 & 0.400 \\
\hline $1 D$ (c.o.g.) & 3.8544 & 0 & 0.860 & 0.396 \\
\hline $2 P$ (c.o.g.) & 4.0576 & 0 & 1.029 & 0.452 \\
\hline $3 S$ & 4.2493 & 0.4840 & 1.174 & 0.512 \\
\hline $2 D$ (c.o.g.) & 4.3281 & 0 & 1.187 & 0.509 \\
\hline $4 S$ & 4.6877 & 0.4562 & 1.460 & 0.615 \\
\hline \hline
\end{tabular}

The overestimated values for the radial excitations could indicate that other corrections might be necessary to fit the whole spectrum with accuracy. One possible solution could be to include the first relativistic correction in the kinetic energy, of order $\mathbf{p}^{4}$, which is supposed to be negative and become more important as radial excitations increase (or other momentdependent corrections). But, as discussed in [62], a good agreement with experimental data can also be obtained with non-relativistic models by choosing a different set of parameters. Maybe in relativistic descriptions, the resulting values for the charm quark mass or the strong coupling constant in this energy scale are more accurate, but in any quarkonium model they should be interpreted more as phenomenological parameters than absolute physical values (as pointed out in Refs. [58, 67]). Introducing a treatment of $\alpha_{s}$ as a "running coupling" probably wouldn't help to fit the higher states, since it would decrease for radial excitations that are in higher energy scales, then the contribution of the negative Coulomb term would be even weaker, so the mass values would probably be higher. If the string tension decreased as the radial excitations increased, it could contribute to lower the mass of these higher states, but this is a delicate subject since it is not simple to justify well this hypothesis. 


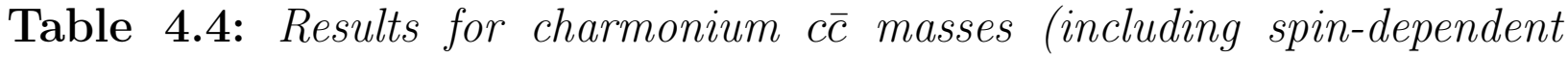
corrections) with model 1 , input of 6 states $(1 S$ and $1 P)$ and modified spinorbit interaction. Parameters are $m_{c}=1.2819, \mathrm{GeV}, \alpha_{s}=0.3289, b=0.2150$ $\mathrm{GeV}^{2}$.

\begin{tabular}{crrrrrrrrr}
\hline \hline$N^{2 S+1} \ell_{J}$ & $\langle T\rangle$ & $\left\langle V_{V}^{(0)}\right\rangle$ & $\left\langle V_{S}^{(0)}\right\rangle$ & $E^{(0)}$ & $M^{(0)}[\mathrm{MeV}]$ & $\left\langle V_{S S}^{(1)}\right\rangle$ & $\left\langle V_{L S}^{(1)}\right\rangle$ & $\left\langle V_{T}^{(1)}\right\rangle$ & $M_{1}^{f}[\mathrm{MeV}]$ \\
\hline $1^{1} S_{0}$ & 364.3 & -292.1 & 436.5 & 508.7 & 3072.5 & -94.4 & 0 & 0 & 2978.1 \\
$1^{3} S_{1}$ & 364.3 & -292.1 & 436.5 & 508.7 & 3072.5 & 31.5 & 0 & 0 & 3104.0 \\
\hline $1^{3} P_{0}$ & 424.9 & -157.9 & 691.7 & 958.7 & 3522.5 & 0 & -72.9 & -24.3 & 3425.4 \\
$1^{3} P_{1}$ & 424.9 & -157.9 & 691.7 & 958.7 & 3522.5 & 0 & -36.4 & 12.1 & 3498.2 \\
$1^{1} P_{1}$ (c.o.g.) & 424.9 & -157.9 & 691.7 & 958.7 & 3522.5 & 0 & 0 & 0 & 3522.5 \\
$1^{3} P_{2}$ & 424.9 & -157.9 & 691.7 & 958.7 & 3522.5 & 0 & 36.4 & -2.4 & 3556.5 \\
\hline $2^{1} S_{0}$ & 513.2 & -181.3 & 845.1 & 1177.0 & 3740.8 & -71.6 & 0 & 0 & 3669.2 \\
$2^{3} S_{1}$ & 513.2 & -181.3 & 845.1 & 1177.0 & 3740.8 & 23.9 & 0 & 0 & 3764.7 \\
\hline $1^{3} D_{1}$ & 507.3 & -115.6 & 898.9 & 1290.6 & 3854.4 & 0 & -32.0 & -3.6 & 3818.9 \\
$1^{3} D_{2}$ & 507.3 & -115.6 & 898.9 & 1290.6 & 3854.4 & 0 & -10.7 & 3.6 & 3847.3 \\
$1^{1} D_{2}$ (c.o.g.) & 507.3 & -115.6 & 898.9 & 1290.6 & 3854.4 & 0 & 0 & 0 & 3854.4 \\
$1^{3} D_{3}$ & 507.3 & -115.6 & 898.9 & 1290.6 & 3854.4 & 0 & 21.3 & -1.0 & 3874.7 \\
\hline $2^{3} P_{0}$ & 579.9 & -123.1 & 1037.0 & 1493.7 & 4057.6 & 0 & -69.0 & -23.0 & 3965.6 \\
$2^{3} P_{1}$ & 579.9 & -123.1 & 1037.0 & 1493.7 & 4057.6 & 0 & -34.5 & 11.5 & 4034.6 \\
$2^{1} P_{1}$ (c.o.g.) & 579.9 & -123.1 & 1037.0 & 1493.7 & 4057.6 & 0 & 0 & 0 & 4057.6 \\
$2^{3} P_{2}$ & 579.9 & -123.1 & 1037.0 & 1493.7 & 4057.6 & 0 & 34.5 & -2.3 & 4089.8 \\
\hline $3^{1} S_{0}$ & 656.7 & -142.3 & 1171.0 & 1685.5 & 4249.3 & -64.6 & 0 & 0 & 4184.7 \\
$3^{3} S_{1}$ & 656.7 & -142.3 & 1171.0 & 1685.5 & 4249.3 & 21.5 & 0 & 0 & 4270.8 \\
\hline $2^{3} D_{1}$ & 653.0 & -97.4 & 1209.0 & 1764.3 & 4328.1 & 0 & -31.3 & -3.5 & 4293.3 \\
$2^{3} D_{2}$ & 653.0 & -97.4 & 1209.0 & 1764.3 & 4328.1 & 0 & -10.4 & 3.5 & 4321.2 \\
$2^{1} D_{2}$ (c.o.g.) & 653.0 & -97.4 & 1209.0 & 1764.3 & 4328.1 & 0 & 0 & 0 & 4328.1 \\
$2^{3} D_{3}$ & 653.0 & -97.4 & 1209.0 & 1764.3 & 4328.1 & 0 & 20.9 & -1.0 & 4348.0 \\
\hline $4^{1} S_{0}$ & 788.5 & -120.8 & 1456.0 & 2123.9 & 4687.7 & -60.9 & 0 & 0 & 4626.8 \\
$4^{3} S_{1}$ & 788.5 & -120.8 & 1456.0 & 2123.9 & 4687.7 & 20.3 & 0 & 0 & 4708.0 \\
\hline \hline & & & & & & & & &
\end{tabular}

\subsubsection{Model 1: Fit with 11 input for comparison with model 2}

After all, it seems that is very difficult (maybe even impossible) to fit at the same time the spin-spin splitting in the $\mathrm{S}$-wave states, the combined spin-orbit and tensor in the $\mathrm{P}$-wave, and also the radial excitations and D-waves with the currently employed model 1.

Introducing a constant term in the potential didn't help, since it only shifts the whole spectrum altogether. Then some stronger modification seemed to be necessary to reach the desired accuracy. 
That is when the model 2 comes into play. We will show in the next Section that with a few changes in the present model we can accommodate reasonably well all the experimental spectrum.

The second model we adopt is the potential model from Refs. [66, 67], which used as input 11 states (in 2005, when it was published, the $h_{c}\left(1^{1} P_{1}\right)$ and $\chi_{c 2}\left(2^{3} P_{2}\right)$ weren't experimentally well-established).

Then in order to compare with the original fit of model 2, we performed a fit with the same input of 11 states, but first using model 1 (with the complete spin-orbit interaction), and using the following range for the three free parameters:

$$
1.1<m_{c}<1.9, \mathrm{GeV}, \quad 0.1<\alpha_{s}<0.7, \quad 0.050<b<0.450, \mathrm{GeV}^{2}
$$

Resulting in:

$$
\chi^{2}=0.0091 \mathrm{GeV}^{2}, \quad m_{c}=1.4803, \mathrm{GeV}, \quad \alpha_{s}=0.5659, \quad b=0.1451 \mathrm{GeV}^{2}
$$

The estimated error (for the 11 states) is $8.7 \mathrm{MeV}$. For comparison we also present in the appendix A the complete results for this set in Tables A.1 and A.2. The mass spectrum compared to experimental data is shown in Figure 4.4 .

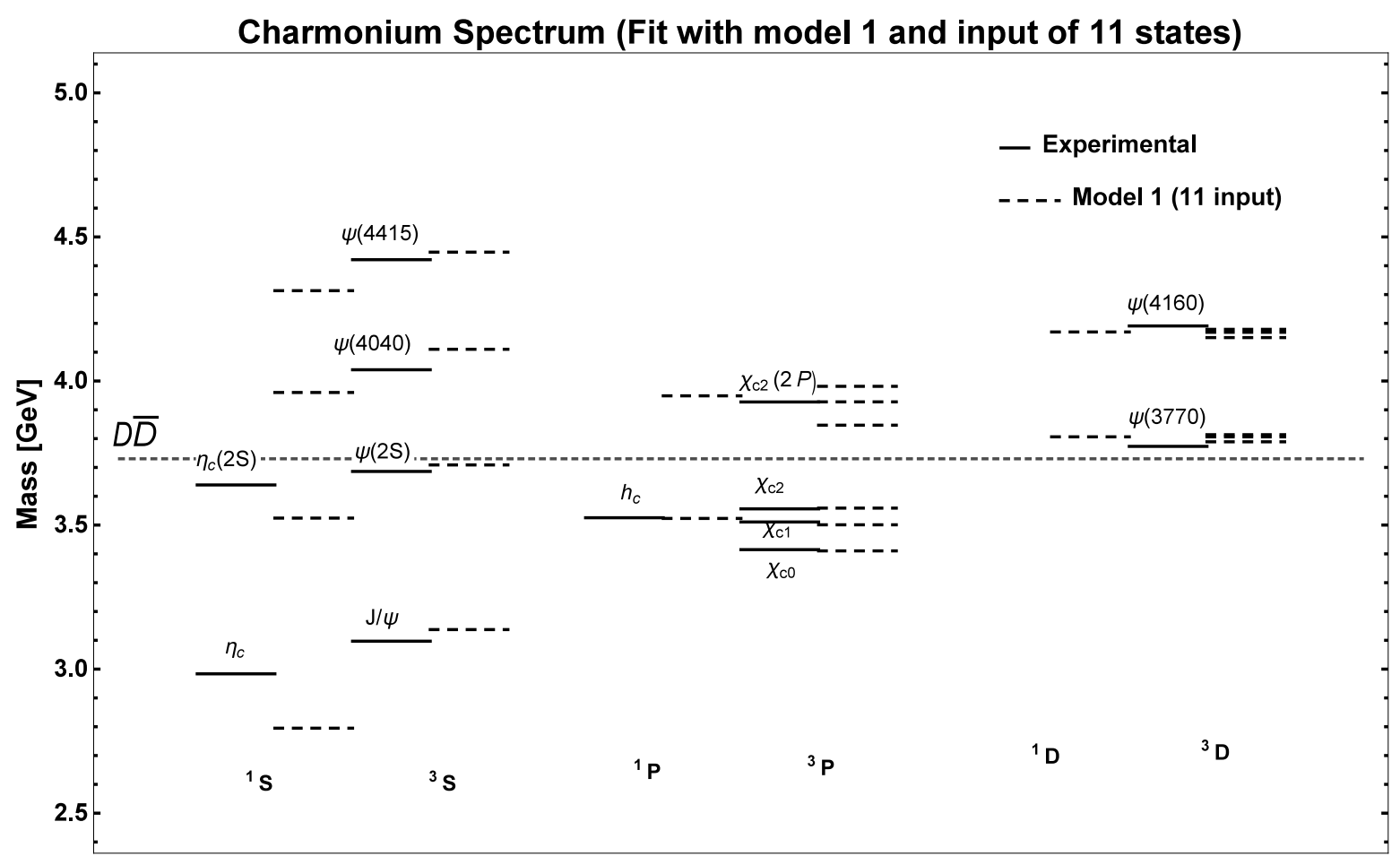

Figure 4.4: Spectrum of charmonium with model 1, input of 11 cic states (without $h_{c}\left(1^{1} P_{1}\right)$ and $\chi_{c 2}\left(2^{3} P_{2}\right)$ ). Parameters are $m_{c}=1.4803 \mathrm{GeV}, \alpha_{s}=$ $0.5659, b=0.1451 \mathrm{GeV}^{2}$

As we can see in the figure, the fit to the four $1 P$ states is much better. The results for the four $1^{3} S$ states, the two D-wave and the one $2 P$ improved, but the $\eta_{c}$ and its first radial excitation are very far from the experimental value.

In particular, the good results for the orbital splitting of the $1 P$ multiplet show that it is possible to fit them with the complete spin-orbit interaction, and at the same time obtain 
reasonable results for higher radial and/or orbital excitations. All that is missing in this fit is a "fine tuning" of the spin-spin interaction, to mend the singlet-triplet hyperfine splitting, because they only affect S-waves (in this model 1). As we show in the next section, the introduction of the gaussian function in the spin-spin interaction (inserted in the zerothorder potential with a new free parameter) is enough to patch everything up.

\subsection{Charmonium: Model 2}

In the second model we consider the zeroth-order potential of the form Coulomb plus linear plus smeared spin-spin. Now we have to separate spin triplet $(\mathrm{S}=1)$ and spin singlet $(\mathrm{S}=0)$ before solving the Schrödinger equation. Then using $\kappa_{s}=-4 / 3, S_{1}=S_{2}=1 / 2$ and $S=0$ or $S=1$ in equation (3.13) we replace the operator $\mathbf{S}_{\mathbf{1}} \cdot \mathbf{S}_{\mathbf{2}}$ by the constant $\left[S(S+1)-S_{1}\left(S_{1}+1\right)-S_{2}\left(S_{2}+1\right)\right] / 2$ it generates when it acts in the wavefunction, resulting:

$$
\begin{array}{ll}
V_{2}^{(0)}(r)=-\frac{4}{3} \frac{\alpha_{s}}{r}+b r-\frac{8 \pi \alpha_{s}}{3 m^{2}}\left(\frac{\sigma}{\sqrt{\pi}}\right)^{3} \mathrm{e}^{-\sigma^{2} r^{2}}, & (S=0) \\
V_{2}^{(0)}(r)=-\frac{4}{3} \frac{\alpha_{s}}{r}+b r+\frac{8 \pi \alpha_{s}}{9 m^{2}}\left(\frac{\sigma}{\sqrt{\pi}}\right)^{3} \mathrm{e}^{-\sigma^{2} r^{2}}, \quad(S=1)
\end{array}
$$

The perturbative spin-dependent potential now is only the spin-orbit and tensor:

$$
\left\langle V_{2, \text { Spin }}^{(1)}(r)\right\rangle=\frac{1}{m^{2}}\left[\left(2 \alpha_{s}\left\langle\frac{1}{r^{3}}\right\rangle-\frac{b}{2}\left\langle\frac{1}{r}\right\rangle\right)\langle\mathbf{L} \cdot \mathbf{S}\rangle+\frac{\alpha_{s}}{3}\left\langle\frac{1}{r^{3}}\right\rangle\left\langle\mathbf{S}_{\mathbf{1 2}}\right\rangle\right]
$$

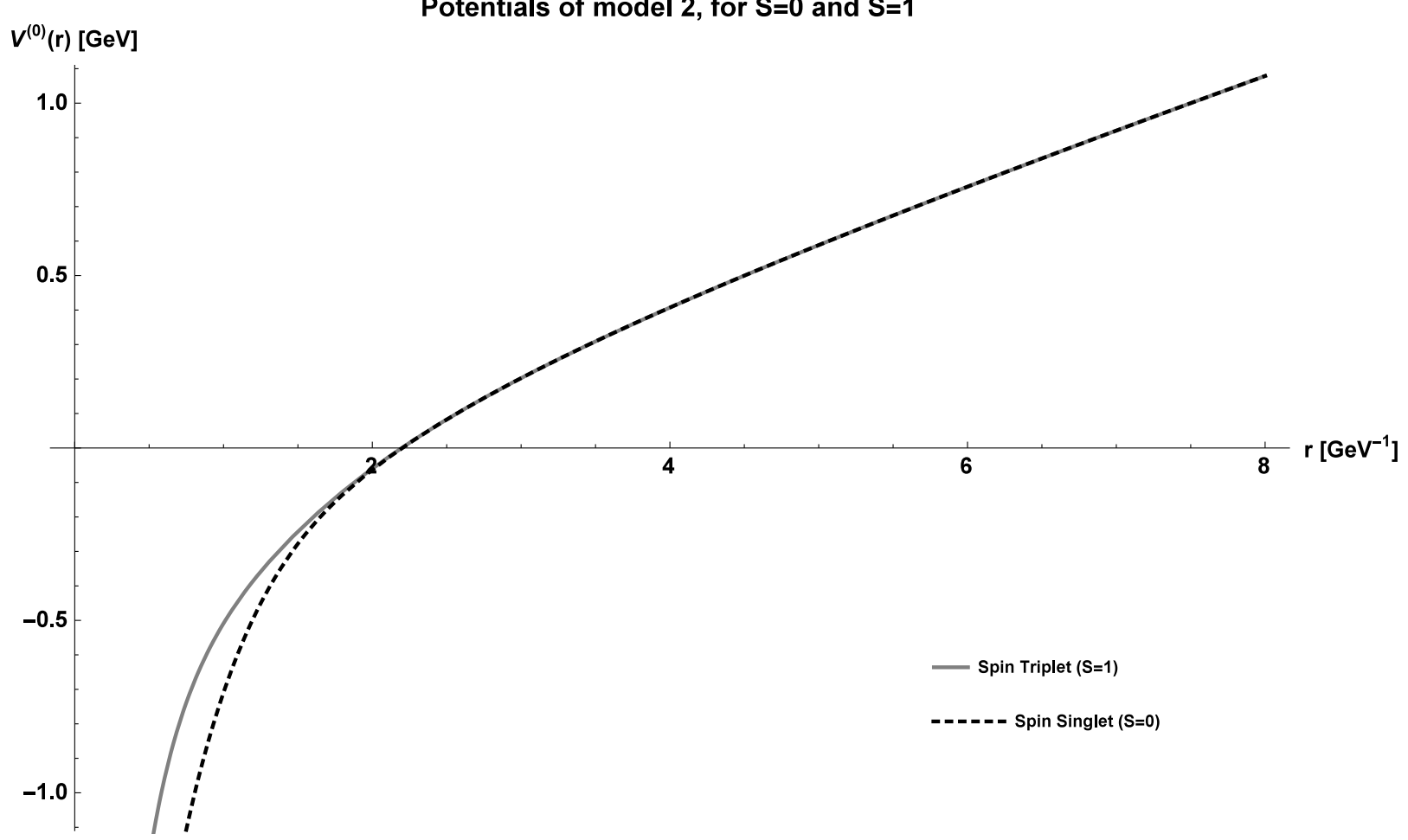

Figure 4.5: Potentials of model 2: Coulomb plus linear plus smeared spinspin, for $S=0$ and $S=1$. Parameters are $\alpha_{s}=0.5285, b=0.1458 \mathrm{GeV}^{2}$, $\sigma=1.1779 \mathrm{GeV}$. 
The original set of parameters of Refs. [66, 67] was obtained with the fit of $11 c \bar{c}$ states with equal statistical weight, resulting:

$$
m_{c}=1.4794 \mathrm{GeV}, \quad \alpha_{s}=0.5461, \quad b=0.1425 \mathrm{GeV}^{2}, \quad \sigma=1.0946 \mathrm{GeV}
$$

Notice how close the original parameters from Refs. [66, 67] are from the ones obtained using model 1 and the same fit with 11 states (in Section 4.1.5). First we used this original set to check the model and reproduce the results. We also performed a fitting with this model 2 and the same 11 states (with updated experimental values from PDG [52] in Section 4.2.2), which can be compared with the equivalent fit using model 1 and the results of references $[66,67]$.

In the following section, we present the updated and most general case using all the 13 states as input in this model 2. The improvement in comparison with the equivalent fit using model 1 (in Section 4.1.1) is very satisfying.

\subsubsection{Model 2: Fit with 13 input}

In order to check our fitting procedure and compare both models with same input, we performed a fit with the same 13 states (with updated experimental values) using this second model. We allowed the four parameters to vary in the following range:

$$
1.1<m_{c}<1.9 \mathrm{GeV}, \quad 0.1<\alpha_{s}<0.7, \quad 0.050<b<0.450 \mathrm{GeV}^{2}, \quad 0.7<\sigma<1.3 \mathrm{GeV}
$$

And the results are also very similar to the originals:

$$
\chi^{2}=0.0063 \mathrm{GeV}^{2}, \quad m_{c}=1.4622 \mathrm{GeV}, \quad \alpha_{s}=0.5202, \quad b=0.1463 \mathrm{GeV}^{2}, \quad \sigma=1.0831 \mathrm{GeV}
$$

The estimated error (for the 13 states) is $6.1 \mathrm{MeV}$. Results for this fit are in Tables 4.5 and 4.6, and the mass spectrum is presented in Figure 4.6. Notice that this set is very similar to the original set of Refs. [66, 67], suggesting that the inclusion of the two new states didn't affect much the fitting. 


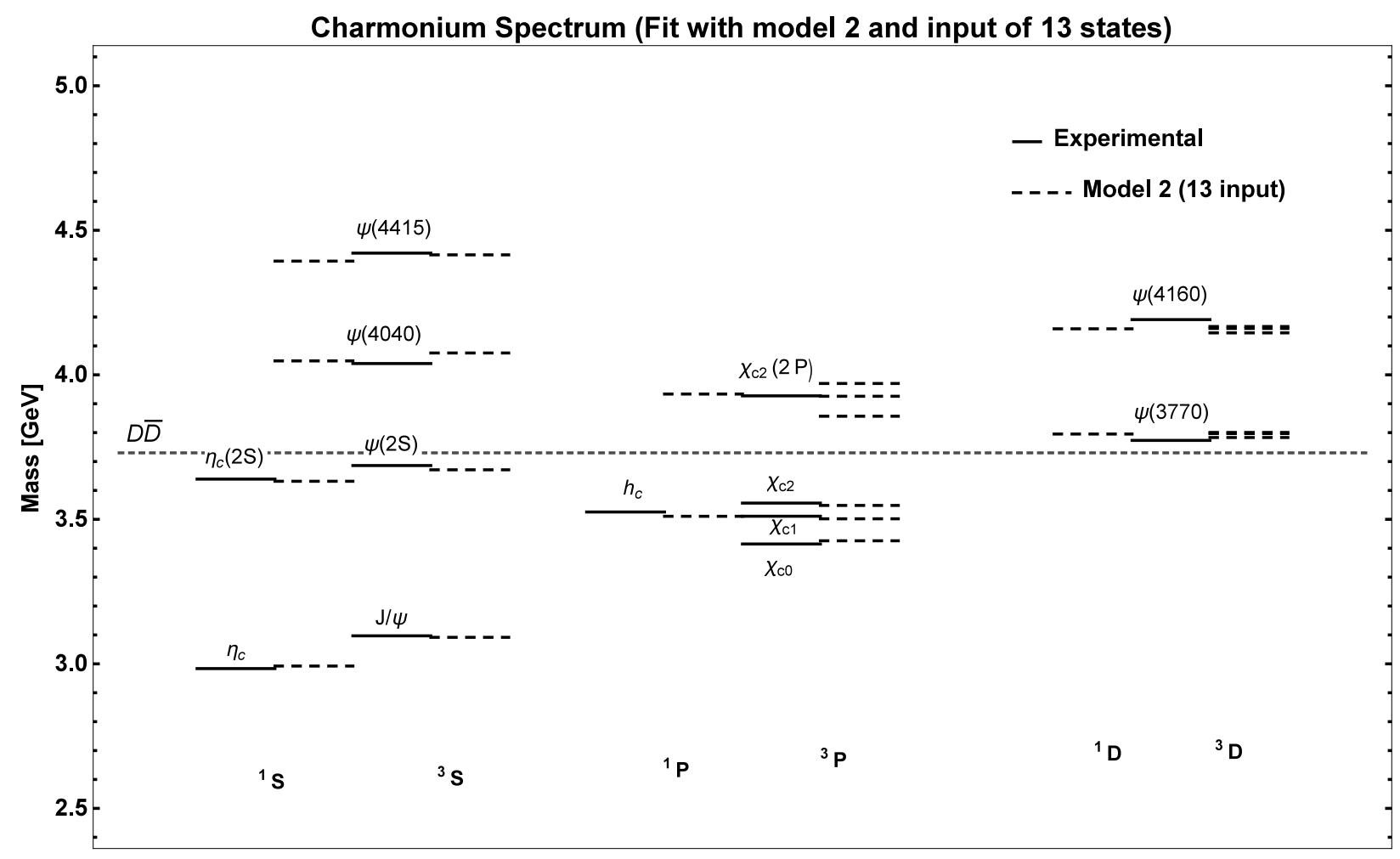

Figure 4.6: Spectrum of charmonium with model 2, input of 13 c $\bar{c}$ states. Parameters are $m_{c}=1.4622 \mathrm{GeV}, \alpha_{s}=0.5202, b=0.1463 \mathrm{GeV}^{2}, \sigma=1.0831$ GeV.

Actually, the prediction for $h_{c}\left(1^{1} P_{1}\right)$ with the original set was already very close to the experimental value (even more if one consider its mass as the c.o.g. of the ${ }^{3} P_{J}$ states, as done in [67] for the calculations where its mass was required). And the inclusion of the quite recently measured $\chi_{c 2}(2 P)$ (see Ref. [56] and references therein) didn't affect much the resulting set, even though the prediction for its mass is a little higher than the experimental value. This could be due to limitations of the model that doesn't take into account effects that might be important in this particular state, or maybe with more statistics from future measures we will discover that its mass is higher than the current value in PDG [52].

In Table 4.5 we present the wavefunction properties. Notice that the inclusion of the spin-spin interaction in the zeroth-order potential creates a small difference between the wavefunction of spin singlet and spin triplet. The spin 0 states have a negative contribution of this interaction in the potential, what causes the close-distances region of the potential (small $r$ coordinate) to be "more negative" (see Figure 4.5) generating states with smaller root mean square radius, higher value of the wavefunction at the origin and higher quark velocity. 


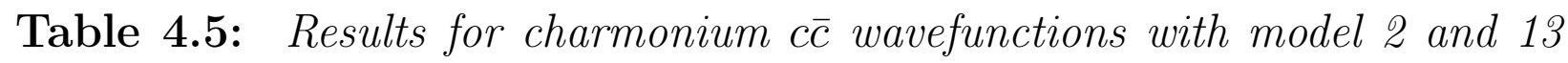
input states. Parameters are $m_{c}=1.4622 \mathrm{GeV}, \alpha_{s}=0.5202, b=0.1463$ $\mathrm{GeV}^{2}, \sigma=1.0831 \mathrm{GeV}$.

\begin{tabular}{crrrr}
\hline \hline$N^{2 S+1} \ell$ & $M^{(0)}[\mathrm{GeV}]$ & $|R(0)|^{2}\left[\mathrm{GeV}^{3}\right]$ & $\left\langle r^{2}\right\rangle^{1 / 2}[\mathrm{fm}]$ & $\left\langle\frac{v^{2}}{c^{2}}\right\rangle$ \\
\hline $1^{1} S$ & 2.9924 & 1.5405 & 0.375 & 0.336 \\
$1^{3} S$ & 3.0917 & 1.1861 & 0.421 & 0.253 \\
\hline $1^{1} P$ & 3.5105 & 0 & 0.678 & 0.257 \\
$1^{3} P$ (c.o.g.) & 3.5191 & 0 & 0.689 & 0.246 \\
\hline $2^{1} S$ & 3.6317 & 0.7541 & 0.839 & 0.308 \\
$2^{3} S$ & 3.6714 & 0.7092 & 0.867 & 0.293 \\
\hline $1^{1} D$ & 3.7951 & 0 & 0.899 & 0.280 \\
$1^{3} D$ (c.o.g.) & 3.7958 & 0 & 0.901 & 0.278 \\
\hline $2^{1} P$ & 3.9334 & 0 & 1.071 & 0.324 \\
$2^{3} P$ (c.o.g.) & 3.9427 & 0 & 1.082 & 0.315 \\
\hline $3^{1} S$ & 4.0481 & 0.6088 & 1.210 & 0.364 \\
$3^{3} S$ & 4.0755 & 0.5914 & 1.230 & 0.357 \\
\hline $2^{1} D$ & 4.1591 & 0 & 1.258 & 0.350 \\
$2^{3} D$ (c.o.g.) & 4.1604 & 0 & 1.261 & 0.348 \\
\hline $4^{1} S$ & 4.3933 & 0.5430 & 1.531 & 0.424 \\
$4^{3} S$ & 4.4150 & 0.5340 & 1.547 & 0.419 \\
\hline \hline
\end{tabular}

In Table 4.6 we present the results for the masses including the spin interactions. Notice that since the spin-spin interaction is no longer treated as perturbation, its contribution to orbitally excited states is no longer zero, specially in $\mathrm{P}$-wave, even tough the wave function at the origin is still compatible with zero (within the assumptions of the numerical method, as discussed in Section 2.6.1). That also causes the spin singlet in orbitally excited states to be different from the spin-average (c.o.g.). This could be a weak spot of this model, because the experimental measures for the $1 P$ states suggest that they should probably be equal, or very close (see Table 4.8 for experimental values). As pointed in Ref. [56], precise measurement of the difference between the c.o.g. of the $1^{3} P_{J}$ states and the singlet $1^{1} P_{1}$ can provide useful information about the spin-dependent interactions in heavy quarks.

If the spin-spin interaction actually has a nonvanishing contribution to orbitally excited states, this model can provide a way to describe it with just this change of the Dirac delta to a gaussian function. 


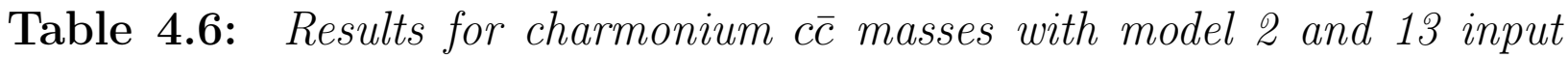
states. Parameters are $m_{c}=1.4622 \mathrm{GeV}, \alpha_{s}=0.5202, b=0.1463 \mathrm{GeV}^{2}$, $\sigma=1.0831 \mathrm{GeV}$.

\begin{tabular}{crrrrrrrrr}
\hline \hline$N^{2 S+1} \ell_{J}$ & $\langle T\rangle$ & $\left\langle V_{V}^{(0)}\right\rangle$ & $\left\langle V_{S}^{(0)}\right\rangle$ & $\left\langle V_{S S}^{(0)}\right\rangle$ & $E^{(0)}$ & $M^{(0)}[\mathrm{MeV}]$ & $\left\langle V_{L S}^{(1)}\right\rangle$ & $\left\langle V_{T}^{(1)}\right\rangle$ & $M_{2}^{f}[\mathrm{MeV}]$ \\
\hline $1^{1} S_{0}$ & 491.9 & -584.4 & 246.2 & -85.6 & 68.1 & 2992.4 & 0 & 0 & 2992.4 \\
$1^{3} S_{1}$ & 370.6 & -504.0 & 279.4 & 21.4 & 167.4 & 3091.7 & 0 & 0 & 3091.7 \\
\hline $1^{3} P_{0}$ & 359.5 & -246.6 & 480.0 & 2.0 & 594.8 & 3519.1 & -63.9 & -29.4 & 3425.8 \\
$1^{3} P_{1}$ & 359.5 & -246.6 & 480.0 & 2.0 & 594.8 & 3519.1 & -32.0 & 14.7 & 3501.8 \\
$1^{1} P_{1}$ & 375.2 & -253.1 & 471.1 & -7.0 & 586.2 & 3510.5 & 0 & 0 & 3510.5 \\
$1^{3} P_{2}$ & 359.5 & -246.6 & 480.0 & 2.0 & 594.8 & 3519.1 & 32.0 & -2.9 & 3548.1 \\
\hline $2^{1} S_{0}$ & 450.6 & -287.3 & 573.8 & -29.7 & 707.4 & 3631.7 & 0 & 0 & 3631.7 \\
$2^{3} S_{1}$ & 428.5 & -281.7 & 590.4 & 9.8 & 747.1 & 3671.4 & 0 & 0 & 3671.4 \\
\hline $1^{3} D_{1}$ & 407.0 & -175.4 & 639.7 & 0.2 & 871.5 & 3795.8 & -8.8 & -3.9 & 3783.1 \\
$1^{3} D_{2}$ & 407.0 & -175.4 & 639.7 & 0.2 & 871.5 & 3795.8 & -2.9 & 3.9 & 3796.7 \\
$1^{1} D_{2}$ & 408.8 & -175.9 & 638.5 & -0.6 & 870.8 & 3795.1 & 0 & 0 & 3795.1 \\
$1^{3} D_{3}$ & 407.0 & -175.4 & 639.7 & 0.2 & 871.5 & 3795.8 & 5.9 & -1.1 & 3800.6 \\
\hline $2^{3} P_{0}$ & 460.4 & -186.2 & 742.1 & 2.2 & 1018.4 & 3942.7 & -59.9 & -26.1 & 3856.7 \\
$2^{3} P_{1}$ & 460.4 & -186.2 & 742.1 & 2.2 & 1018.4 & 3942.7 & -29.9 & 13.0 & 3925.8 \\
$2^{1} P_{1}$ & 474.4 & -190.8 & 733.1 & -7.5 & 1009.1 & 3933.4 & 0 & 0 & 3933.4 \\
$2^{3} P_{2}$ & 460.4 & -186.2 & 742.1 & 2.2 & 1018.4 & 3942.7 & 29.9 & -2.6 & 3970.0 \\
\hline $3^{1} S_{0}$ & 532.8 & -215.4 & 826.5 & -20.1 & 1123.8 & 4048.1 & 0 & 0 & 4048.1 \\
$3^{3} S_{1}$ & 521.9 & -215.3 & 837.7 & 6.9 & 1151.2 & 4075.5 & 0 & 0 & 4075.5 \\
\hline $2^{3} D_{1}$ & 508.6 & -145.8 & 873.0 & 0.3 & 1236.1 & 4160.4 & -11.6 & -3.7 & 4145.1 \\
$2^{3} D_{2}$ & 508.6 & -145.8 & 873.0 & 0.3 & 1236.1 & 4160.4 & -3.9 & 3.7 & 4160.2 \\
$2^{1} D_{2}$ & 511.3 & -146.5 & 871.0 & -1.0 & 1234.8 & 4159.1 & 0 & 0 & 4159.1 \\
$2^{3} D_{3}$ & 508.6 & -145.8 & 873.0 & 0.3 & 1236.1 & 4160.4 & 7.7 & -1.1 & 4167.1 \\
\hline $4^{1} S_{0}$ & 620.4 & -179.5 & 1044.0 & -15.8 & 1469.0 & 4393.3 & 0 & 0 & 4393.3 \\
$4^{3} S_{1}$ & 613.2 & -180.6 & 1053.0 & 5.6 & 1490.7 & 4415.0 & 0 & 0 & 4415.0 \\
\hline \hline
\end{tabular}




\subsubsection{Model 2: Fit with 11 input for comparison with model 1}

Just to check our fitting procedure and to compare both models with the same input and the original parameter set from Refs. [66, 67], we also performed a fit with the same 11 states (with updated experimental values) using this second model. We allowed the four parameters to vary same range as used in the fit with 13 states.

And the results are also very similar to the originals:

$\chi^{2}=0.0041 \mathrm{GeV}^{2}, \quad m_{c}=1.4685 \mathrm{GeV}, \quad \alpha_{s}=0.5285, \quad b=0.1458 \mathrm{GeV}^{2}, \quad \sigma=1.1779 \mathrm{GeV}$

The estimated error (for the 11 states) is $5.8 \mathrm{MeV}$. Results for this fit are in Tables A.3 and A.4 in the appendix A. In Figure 4.7 we show the mass spectrum. Notice that it is very similar to the one with 13 input (also with the original work from Refs. [66, 67]). Actually, if one compare mainly the states below the threshold, the results predicted with model 2 and input of 11 states are a little better than the ones of model 2 using 13 input. But since we want to compare model 2 and the fit of model 1 with 13 input (because model 1 with 11 input had bad $\eta_{c}$ predictions), then we will use the results of model 2 with 13 input as its representative in the next section.

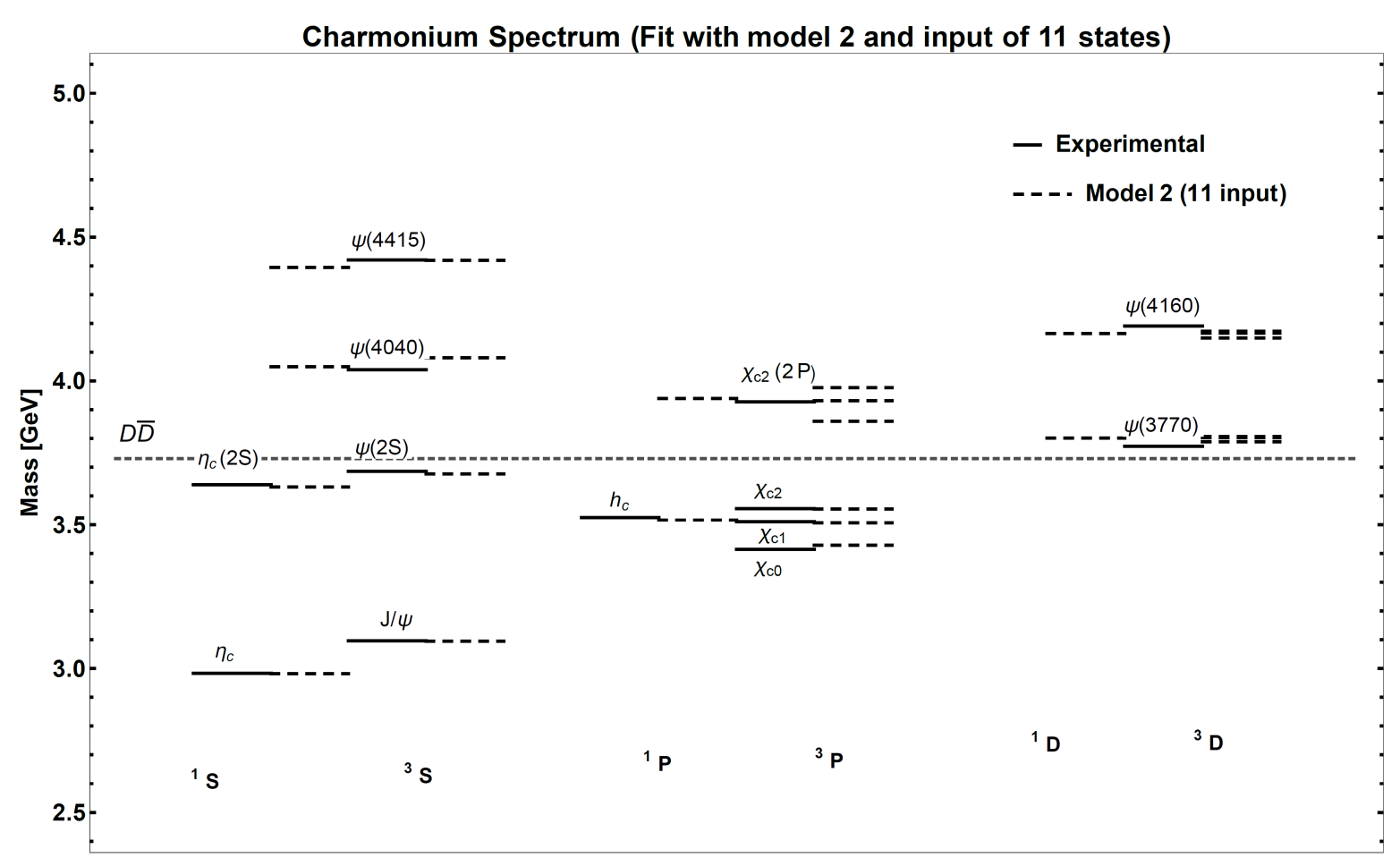

Figure 4.7: Spectrum of charmonium with model 2, input of 11 c $\bar{c}$ states (without $h_{c}\left(1^{1} P_{1}\right)$ and $\chi_{c 2}\left(2^{3} P_{2}\right)$ ). Parameters are $m_{c}=1.4685 \mathrm{GeV}, \alpha_{s}=$ $0.5285, b=0.1458 \mathrm{GeV}^{2}, \sigma=1.1779 \mathrm{GeV}$. 


\subsection{Charmonium: Comparison with Experimental Data}

In Table 4.7 we list the properties and results of parameters for each of the fittings discussed in the previous sections.

Table 4.7: Parameters used in all the different models and FITs.

\begin{tabular}{|c|c|c|c|c|c|c|c|c|}
\hline$m_{c}[\mathrm{GeV}]$ & $\alpha_{s}$ & $b\left[\mathrm{GeV}^{2}\right]$ & $\sigma[\mathrm{GeV}]$ & $V_{0}[\mathrm{GeV}]$ & Model & Input [52] & $\chi^{2}\left[\mathrm{GeV}^{2}\right]$ & $\frac{\text { Error }}{\text { Input }}[\mathrm{MeV}]$ \\
\hline 1.4115 & 0.4116 & 0.1517 & - & - & 1 & 13 & 0.0132 & 8.8 \\
\hline 1.7378 & 0.4066 & 0.15945 & - & -0.5871 & $1 V_{0}$ & 13 & 0.0124 & 8.9 \\
\hline 1.3465 & 0.3796 & 0.1857 & - & - & 1 & $6(1 S 1 P)$ & 0.0028 & 8.6 \\
\hline 1.2819 & 0.3289 & 0.2150 & - & - & $1 \mathrm{LS}$ & $6(1 \mathrm{~S} 1 \mathrm{P})$ & 0.0004 & 3.1 \\
\hline 1.4803 & 0.5659 & 0.1425 & - & - & 1 & $11(2015)$ & 0.0091 & 8.7 \\
\hline 1.4794 & 0.5461 & 0.1451 & 1.0946 & - & $2[66,67]$ & $11(2005)$ & - & - \\
\hline 1.4622 & 0.5202 & 0.1463 & 1.0831 & - & 2 & 13 & 0.0063 & 6.1 \\
\hline 1.4685 & 0.5285 & 0.1458 & 1.1779 & - & 2 & 11 (2015) & 0.0041 & 5.8 \\
\hline
\end{tabular}

In Table 4.8 we compare the experimental data with the results of two fits of Model 1 , the one in section 4.1 .1 with 13 input, the one with modified spin-orbit interaction and input of 6 states (1S and 1P) in Section 4.1.4, and one fit of Model 2, with input of 13 states in Section 4.2.1.

We adopt the nomenclature and data from the 2014 PDG with update from 2015 [52]. So far there are 13 states well-established as $c \bar{c}$. 
Table 4.8: Comparison of charmonium cē experimental data and theoretical calculations from Sections 4.1.1 and 4.1.4 of model 1 and 4.2.1 of model 2.

\begin{tabular}{|c|c|c|c|c|c|c|}
\hline$N^{2 S+1} \ell_{J}$ & $M_{1}^{f}$ Tab. 4.2 & $M_{1}^{f}$ Tab. 4.4 & $M_{2}^{f}[\mathrm{MeV}]$ Tab. 4.6 & $\operatorname{Exp}[52][\mathrm{MeV}]$ & Meson & $J^{P C}$ \\
\hline $1^{1} S_{0}$ & 2984.5 & 2978.1 & 2992.4 & $2983.6 \pm 0.6$ & $\eta_{c}(1 S)$ & $0^{-+}$ \\
\hline $1^{3} S_{1}$ & 3141.7 & 3104.0 & 3091.7 & $3096.916 \pm 0.011$ & $J / \psi(1 S)$ & $1^{--}$ \\
\hline $1^{3} P_{0}$ & 3440.4 & 3425.4 & 3425.8 & $3414.75 \pm 0.31$ & $\chi_{c 0}(1 P)$ & $0^{++}$ \\
\hline $1^{3} P_{1}$ & 3494.7 & 3498.2 & 3501.8 & $3510.66 \pm 0.07$ & $\chi_{c 1}(1 P)$ & $1^{++}$ \\
\hline $1^{1} P_{1}$ & 3504.1 & 3522.5 & 3510.5 & $3525.38 \pm 0.11$ & $h_{c}(1 P)^{\dagger}$ & $1^{+-}$ \\
\hline $1^{3} P_{2}$ & 3522.4 & 3556.5 & 3548.1 & $3556.20 \pm 0.09$ & $\chi_{c 2}(1 P)$ & $2^{++}$ \\
\hline $1 P$ (c.o.g.) & $(3504.1)$ & $(3522.5)$ & $(3519.1)$ & $(3525.303)$ & - & - \\
\hline $2^{1} S_{0}$ & 3585.1 & 3669.2 & 3631.7 & $3639.2 \pm 1.2$ & $\eta_{c}(2 S)$ & $0^{-+}$ \\
\hline $2^{3} S_{1}$ & 3688.0 & 3764.7 & 3671.4 & $3686.109_{-0.014}^{+0.012}$ & $\psi(2 S)$ & $1^{--}$ \\
\hline $1^{3} D_{1}$ & 3773.1 & 3818.9 & 3783.1 & $3773.15 \pm 0.33$ & $\psi(3770)$ & $1^{--}$ \\
\hline $1^{3} D_{2}$ & 3775.6 & 3847.3 & 3796.7 & - & - & $2^{--}$ \\
\hline $1^{1} D_{2}$ & 3778.8 & 3854.4 & 3795.1 & - & - & $2^{-+}$ \\
\hline $1^{3} D_{3}$ & 3774.3 & 3874.7 & 3800.6 & - & - & $3^{--}$ \\
\hline $1 D$ (c.o.g.) & $(3778.8)$ & $(3854.4)$ & $(3795.8)$ & - & - & - \\
\hline $2^{3} P_{0}$ & 3866.1 & 3965.6 & 3856.7 & - & $*$ & $0^{++}$ \\
\hline $2^{3} P_{1}$ & 3917.9 & 4034.6 & 3925.8 & - & - & $1^{++}$ \\
\hline $2^{1} P_{1}$ & 3928.4 & 4057.6 & 3933.4 & - & - & $1^{+-}$ \\
\hline $2^{3} P_{2}$ & 3947.2 & 4089.8 & 3970.0 & $3927.2 \pm 2.6$ & $\chi_{c 2}(2 P)^{\dagger}$ & $2^{++}$ \\
\hline $2 P$ (c.o.g.) & (3928.4) & $(4057.6)$ & $(3942.7)$ & - & - & - \\
\hline $3^{1} S_{0}$ & 4001.6 & 4184.7 & 4048.1 & - & - & $0^{-+}$ \\
\hline $3^{3} S_{1}$ & 4090.1 & 4270.8 & 4075.5 & $4039 \pm 1$ & $\psi(4040)$ & $1^{--}$ \\
\hline $2^{3} D_{1}$ & 4140.0 & 4293.3 & 4145.1 & $4191 \pm 5$ & $\psi(4160)$ & $1^{--}$ \\
\hline $2^{3} D_{2}$ & 4148.0 & 4321.2 & 4160.2 & - & - & $2^{--}$ \\
\hline $2^{1} D_{2}$ & 4146.0 & 4328.1 & 4159.1 & - & - & $2^{-+}$ \\
\hline $2^{3} D_{3}$ & 4147.2 & 4348.0 & 4167.1 & - & - & $3^{--}$ \\
\hline $2 D$ (c.o.g.) & $(4146.0)$ & $(4160.4)$ & $(4158.9)$ & - & - & - \\
\hline $4^{1} S_{0}$ & 4351.7 & 4626.8 & 4393.3 & - & - & $0^{-+}$ \\
\hline $4^{3} S_{1}$ & 4433.0 & 4708.0 & 4415.0 & $4421 \pm 4$ & $\psi(4415)$ & $1^{--}$ \\
\hline
\end{tabular}

${ }^{\dagger} h_{c}(1 P)$ and $\chi_{c 2}(2 P)$ weren't experimentally well-established when Ref. [67] (of model 2) was published, so these states were not used as input in that work.

* In the 2014 edition of the PDG the $X(3915)$ was assigned to this $c \bar{c}$ state, the $\chi_{c 0}(2 P)$, but due to many reasons [54] it has been demoted from that position. The $X(3915)$ still has a status of exotic resonance and there might be some evidence of the "real" $\chi_{c 0}(2 P)$ in Belle and BaBar recent data that indicates its mass could be around $3837.6 \pm 11.5 \mathrm{MeV}$ [53], which is in better agreement with quarkonium models. A recent example of the $X(3915)$, now denoted by $\chi_{c 0}(3915)$, interpreted as a diquark-antidiquark tetraquark $[c s][\bar{c} \bar{s}]$ can be found in Ref. [55]. 


\section{Chapter 5}

\section{Diquarks}

Diquarks have gained popularity in the past decade since many recent works on exotics adopt the diquark-antidiquark picture of tetraquarks (with different assumptions, methods and flavor of the constituents). Also in baryon spectroscopy diquarks have been used to explain missing resonances of 3-body model predictions, by factorizing the baryon as a diquark-quark system. For example, in Ref. [82] they factorize the baryon problem with two heavy quarks and one strange quark in two consecutive 2-body problems: first the two heavy quarks bound as a diquark, and then the diquark and strange quark bound as a baryon. Another example is the quark-diquark model of nucleons, in Ref. [83].

In this chapter we present our calculations for heavy diquarks composed by two charm quarks $c c$ (which are equivalent for antidiquarks $\bar{c} \bar{c}$ in our framework). We use the same models from charmonium, except that due to the different color structure, the color factor now is $\kappa_{s}=-\frac{2}{3}$, which corresponds to the attractive antitriplet color state, as discussed in Section 2.4 .

There is one important consideration regarding the fact that two charm quarks together is a systems of identical fermions, therefore the total wavefunction has to be antisymmetric to satisfy the Pauli exclusion principle. The flavor part is symmetric (both charm quarks). The spatial part parity will depend on the orbital angular momentum, which contributes with a factor $(-1)^{\ell}$ (this time there is no extra $(-1)$ factor, because the quarks are both particles, or both antiparticles). The color part is antisymmetric in the antitriplet color state (attractive), and symmetric in the sextet color state (repulsive).

Since we adopt the antitriplet color state, we have that for S-wave diquark $(\ell=0)$ the spatial part is symmetric too, which implies that the spin state also has to be symmetric for the total wavefunction of the diquark to be antisymmetric. Then, S-wave $c c$ diquarks in antitriplet color state can only be in the spin triplet state $(S=1)$, which is symmetric.

In general, if we consider the diquark in the antitriplet color state, when the orbital part is symmetric $(\ell=2 k, k=0,1,2, \ldots)$, the spin state has to be symmetric too (spin triplet, $S=1)$, and when the orbital part is antisymmetric $(\ell=2 k+1, k=0,1,2, \ldots)$, the spin state has to be antisymmetric too (spin singlet, $S=0$ ). A P-wave diquark in antitriplet color configuration, for example, has to be in the spin singlet $S=0$, and so on.

Some models adopt trial wavefunctions that also have parity related to the radial excitations, and the parity restrictions can be different. In general, from the relativistic point of view, diquarks with spin 0 have a scalar structure, and diquarks with spin 1 have an axial-vector structure. Since we adopt a simplified description of non-relativistic quantum mechanics, we will treat angular momentum considering $S, \ell$ and $J$ (total spin, orbital and total angular momentum, respectively) as good quantum numbers, as we discussed in 
Chapter 3.

Notice that going from the color factor $-4 / 3$ (for quark-antiquark meson in the singlet color state) to the color factor $-2 / 3$ (for quark-quark in the antitriplet color state) we introduced a factor $1 / 2$ in this change. Because of that, it is very common to adopt a general rule of extending this factor $1 / 2$ to the whole potential describing the quark-quark interaction. This rule is inspired in the interactions inside baryons, where two quarks can also be considered to form an antitriplet-color diquark, which then can interact with the third quark and form a singlet color baryon. Since this seems to give satisfactory results in baryon spectroscopy, it has been also extended to diquarks inside tetraquarks. The prescription is the following:

$$
V_{q q}=\frac{1}{2} V_{q \bar{q}}
$$

So based on that, many authors with different tetraquark models (for instance, Refs. $[84,85]$ also divide the meson potential by 2 in order to adapt it to the diquark case. Then in our models, besides the change in the color factor, the string tension $b$ would also be divided by 2 (and even any constant term like $V_{0}$ ).

The gaussian in our second model is automatically divided by two because it is proportional to the color factor. In fact all the spin corrections of vector origin (due to one gluon exchange) are proportional to the color factor. However the second term of the spin-orbit interaction (Thomas precession), which is proportional to the string tension $b$ and comes from the assumption of scalar structure of confinement, is not proportional to the color factor, what might suggest again that one should also divide the string tension by 2 . This way, not just the zeroth-order potential, but also the first-order spin dependent potential has a overall factor $1 / 2$ if compared to the meson one (in model 1 and 2).

This prescription could be questionable. The color factor due to one gluon exchange is very clear, but the origin of the string tension parameter is mainly phenomenological. In some models (for instance, Refs. [62, 84]) it is assumed that part of the confinement comes from a vector contribution, so applying the factor $1 / 2$ is better justified.

As discussed along the text, we are interested in building a tetraquark composed of diquarks in the lowest level of the attractive antitriplet-color configuration, which is more likely to form a compact and stable diquark. Then it should be an $1 S$ state, with no orbital nor radial excitation. Taking into account the antisymmetry of identical quarks, it should be a spin triplet $(S=1)$, so it is the diquark state analogous to the $J / \psi\left(1^{3} S_{1}\right)$.

Just to add a little more information, we will also calculate the P-wave diquark (which needs to be in the spin singlet state $(S=0)$ to respect the antisymmetry), and the first radial excitation of both $1 S$ and $1 P$.

We have chosen three of the results for the charmonium parameters and models that seem more appropriate to adapt to the diquark and tetraquark context, which will be discussed in the following sections.

\section{$5.1 \quad$ Model 1}

Since we are going to build the tetraquark with diquarks in the ground state with total spin 1 , analogous to the $J / \psi\left(1^{3} S_{1}\right)$, we can't choose any parameter set that gives very bad results for the spin-spin splitting of the S-wave states, and it would also be desirable that the splittings of the orbitally excited states and of the radial excitations were acceptable (even though in higher charmonia the effects that are being neglected in both models could have a more substantial contribution). 
With that in mind, we can already discard the fit of model 1 with 11 states (made just to compare how the model 2 fixes the spin-spin problem).

The fits with 13 states (with and without a constant term $V_{0}$ ) weren't bad in general aspects, but none of the spin-dependent splittings were very accurate. Since the use of a constant term $V_{0}$ only increased the charm quark mass without any improvement on the agreement with experimental data, we will discard this set.

Then as our first choice we will use the first fit with model 1 and $13 c \bar{c}$ as input, because it could be reasonable to study of the whole spectrum of the tetraquark, and also a good example of the complete model 1, even though it is not the best choice to get the ground state diquark.

First we use the common prescription of equation (5.1), so besides changing the color factor to $\kappa_{s}=-\frac{2}{3}$, we also divide the string tension by 2 (from the meson potential, so we write explicitly $\left.b_{c \bar{c}}\right)$ :

$$
V_{1, c c}^{(0)}=\frac{1}{2} V_{1, c \bar{c}}^{(0)} \quad \rightarrow \quad V_{1, c c}^{(0)}=-\frac{2}{3} \frac{\alpha_{s}}{r}+\left(\frac{b_{c \bar{c}}}{2}\right) r
$$

The strong coupling constant is considered to be the same used in $c \bar{c}$ charmonium, and the first-order (spin-dependent) potential for the quark-quark interaction will be:

$$
\left\langle V_{1, S p i n, c c}^{(1)}(r)\right\rangle=\frac{1}{m^{2}}\left[\frac{4 \alpha_{s}}{9}|R(0)|^{2}\left\langle\mathbf{S}_{\mathbf{1}} \cdot \mathbf{S}_{\mathbf{2}}\right\rangle+\left(\alpha_{s}\left\langle\frac{1}{r^{3}}\right\rangle-\frac{\left(b_{c \bar{c}} / 2\right)}{2}\left\langle\frac{1}{r}\right\rangle\right)\langle\mathbf{L} \cdot \mathbf{S}\rangle+\frac{\alpha_{s}}{6}\left\langle\frac{1}{r^{3}}\right\rangle\left\langle\mathbf{S}_{\mathbf{1 2}}\right\rangle\right]
$$

In Tables 5.1 and 5.2 we present the results for the diquark wavefunctions and masses, respectively, using the complete model 1 and the set of parameters obtained with $13 c \bar{c}$ as input.

Table 5.1: Results for diquark cc wavefunctions with model 1. Parameters from charmonium in Sec. 4.1.1: $m_{c}=1.4155 \mathrm{GeV}, \alpha_{s}=0.4116$ and $b=$ $b_{c \bar{c}} / 2=0.1517 / 2$ Ge $V^{2}$.

\begin{tabular}{lrrrr}
\hline \hline$N \ell$ & $M^{(0)}[\mathrm{GeV}]$ & $|R(0)|^{2}\left[\mathrm{GeV}^{3}\right]$ & $\left\langle r^{2}\right\rangle^{1 / 2}[\mathrm{fm}]$ & $\left\langle\frac{v^{2}}{c^{2}}\right\rangle$ \\
\hline $\mathbf{1 S}$ & $\mathbf{3 . 0 8 4 2}$ & $\mathbf{0 . 2 6 2 4}$ & $\mathbf{0 . 6 1 3}$ & $\mathbf{0 . 1 2 2}$ \\
\hline $1 P$ & 3.2982 & 0 & 0.926 & 0.144 \\
\hline $2 S$ & 3.4042 & 0.2022 & 1.164 & 0.174 \\
\hline $2 P$ & 3.5557 & 0 & 1.412 & 0.197 \\
\hline \hline
\end{tabular}


Table 5.2: Results for diquark cc masses with model 1. Parameters from charmonium in Sec. 4.1.1: $m_{c}=1.4155 \mathrm{GeV}, \alpha_{s}=0.4116$ and $b=b_{c \bar{c}} / 2=$ $0.1517 / 2 \mathrm{GeV}^{2}$.

\begin{tabular}{crrrrrrrrr}
\hline \hline$N^{2 S+1} \ell_{J}$ & $\langle T\rangle$ & $\left\langle V_{V}^{(0)}\right\rangle$ & $\left\langle V_{S}^{(0)}\right\rangle$ & $E^{(0)}$ & $M^{(0)}[\mathrm{MeV}]$ & $\left\langle V_{S S}^{(1)}\right\rangle$ & $\left\langle V_{L S}^{(1)}\right\rangle$ & $\left\langle V_{T}^{(1)}\right\rangle$ & $M_{1}^{f}[\mathrm{MeV}]$ \\
\hline $\mathbf{1}^{\mathbf{3}} \mathbf{S}_{\mathbf{1}}$ & $\mathbf{1 7 2 . 4}$ & $-\mathbf{1 3 2 . 0}$ & $\mathbf{2 1 2 . 9}$ & $\mathbf{2 5 3 . 3}$ & $\mathbf{3 0 8 4 . 2}$ & $\mathbf{6 . 0}$ & $\mathbf{0}$ & $\mathbf{0}$ & $\mathbf{3 0 9 0 . 2}$ \\
\hline $1^{1} P_{1}$ & 203.6 & -71.9 & 335.4 & 467.2 & 3298.2 & 0 & 0 & 0 & 3298.2 \\
\hline $2^{3} S_{1}$ & 246.1 & -82.5 & 409.7 & 573.3 & 3404.2 & 4.6 & 0 & 0 & 3408.8 \\
\hline $2^{1} P_{1}$ & 279.0 & -56.1 & 501.9 & 724.8 & 3555.7 & 0 & 0 & 0 & 3555.7 \\
\hline \hline
\end{tabular}

Notice that the factor $1 / 2$ in the potential caused an attenuation of the interaction. In comparison with the results for charmonium mesons with the same model and parameters, the diquarks have lower mass, value of the wavefunction at the origin, velocity of constituents, spin-dependent interactions and higher root mean square radius. The ground state $1^{3} S$ is very similar to the $J / \psi$, but the radial and orbital excitations grow slower. These general remarks are similar in the next two models for diquarks.

\subsection{Model 1 with modified Spin-Orbit}

Within the remaining sets of model 1 for charmonium, we can also discard the fit with only 6 input states $(1 S$ and $1 P)$ and the complete model 1, which didn't fit well neither the $1 S$ splitting nor the $1 P$ splitting.

But the same fit (with 6 input and model 1) where the term proportional to the string tension in the spin-orbit interaction was removed, actually resulted in a much better agreement with experimental values of these 6 states, and it also has the interesting aspect of low value of the charm quark mass and strong coupling constant $\alpha_{s}$. So even though the higher excitations were overestimated, this is a good set to get the diquark mass, since we are interested in the low lying diquarks. Also, the spin-orbit and tensor interactions vanish for these particular 4 states of diquark we are calculating, so the modification of the spin-orbit interaction will only affect the tetraquark splitting later, when we employ this version of model 1 to describe it.

In Tables 5.3 and 5.4 we present the results for the diquark wavefunctions and masses, respectively, using the model 1 with modified spin-orbit and the set of parameters obtained with it, using $6 c \bar{c}$ as input $(1 S$ and $1 P)$. Notice that mainly due to the low charm quark mass, the diquark mass is smaller than in the previous section. 
Table 5.3: Results for diquark cc wavefunctions with model 1 and modified spin-orbit. Parameters from charmonium in Sec. 4.1.4: $m_{c}=1.2819 \mathrm{GeV}$, $\alpha_{s}=0.3289, b=b_{c \bar{c}} / 2=0.2150 / 2 G e V^{2}$.

\begin{tabular}{rrrrr}
\hline \hline$N \ell$ & $M^{(0)}[\mathrm{GeV}]$ & $|R(0)|^{2}\left[\mathrm{GeV}^{3}\right]$ & $\left\langle r^{2}\right\rangle^{1 / 2}[\mathrm{fm}]$ & $\left\langle\frac{v^{2}}{c^{2}}\right\rangle$ \\
\hline $\mathbf{1 S}$ & $\mathbf{2 . 9 4 9 8}$ & $\mathbf{0 . 2 5 1 5}$ & $\mathbf{0 . 5 9 4}$ & $\mathbf{0 . 1 5 6}$ \\
\hline $1 P$ & 3.2041 & 0 & 0.870 & 0.198 \\
\hline $2 S$ & 3.3469 & 0.2120 & 1.095 & 0.240 \\
\hline $2 P$ & 3.5333 & 0 & 1.315 & 0.277 \\
\hline \hline
\end{tabular}

Table 5.4: Results for diquark cc masses with model 1 with modified spinorbit and parameters from charmonium in Sec. 4.1.4: $m_{c}=1.2819 \mathrm{GeV}, \alpha_{s}=$ $0.3289, b=b_{c \bar{c}} / 2=0.2150 / 2 G e V^{2}$.

\begin{tabular}{crrrrrrrrr}
\hline \hline$N^{2 S+1} \ell_{J}$ & $\langle T\rangle$ & $\left\langle V_{V}^{(0)}\right\rangle$ & $\left\langle V_{S}^{(0)}\right\rangle$ & $E^{(0)}$ & $M^{(0)}[\mathrm{MeV}]$ & $\left\langle V_{S S}^{(1)}\right\rangle$ & $\left\langle V_{L S}^{(1)}\right\rangle$ & $\left\langle V_{T}^{(1)}\right\rangle$ & $M_{1}^{f}[\mathrm{MeV}]$ \\
\hline $\mathbf{1}^{\mathbf{3}} \mathbf{S}_{\mathbf{1}}$ & $\mathbf{2 0 0 . 2}$ & $\mathbf{- 1 0 7 . 3}$ & $\mathbf{2 9 3 . 1}$ & $\mathbf{3 8 6 . 0}$ & $\mathbf{2 9 4 9 . 8}$ & $\mathbf{5 . 6}$ & $\mathbf{0}$ & $\mathbf{0}$ & $\mathbf{2 9 5 5 . 4}$ \\
\hline $1^{1} P_{1}$ & 254.0 & -60.9 & 447.1 & 640.2 & 3204.1 & 0 & 0 & 0 & 3204.1 \\
\hline $2^{3} S_{1}$ & 307.5 & -69.7 & 545.3 & 783.1 & 3346.9 & 4.7 & 0 & 0 & 3351.6 \\
\hline $2^{1} P_{1}$ & 355.2 & -48.0 & 662.3 & 969.4 & 3533.3 & 0 & 0 & 0 & 3533.3 \\
\hline \hline
\end{tabular}

\subsection{Model 2}

In the second model we consider the zeroth-order potential of the form Coulomb plus linear plus smeared spin-spin. Again we separate spin triplet $(\mathrm{S}=1)$ and spin singlet $(\mathrm{S}=0)$, before solving the Schrödinger equation. But now we use $\kappa_{s}=-\frac{2}{3}$, and also divide the string tension by 2 :

$$
\begin{array}{ll}
V_{2, c c}^{(0)}(r)=-\frac{2}{3} \frac{\alpha_{s}}{r}+\left(\frac{b_{c \bar{c}}}{2}\right) r-\frac{4 \pi \alpha_{s}}{3 m^{2}}\left(\frac{\sigma}{\sqrt{\pi}}\right)^{3} \mathrm{e}^{-\sigma^{2} r^{2}}, \quad(S=0) \\
V_{2, c c}^{(0)}(r)=-\frac{2}{3} \frac{\alpha_{s}}{r}+\left(\frac{b_{c \bar{c}}}{2}\right) r+\frac{4 \pi \alpha_{s}}{9 m^{2}}\left(\frac{\sigma}{\sqrt{\pi}}\right)^{3} \mathrm{e}^{-\sigma^{2} r^{2}}, \quad(S=1)
\end{array}
$$

The perturbative spin-dependent potential now is only the spin-orbit and tensor:

$$
\left\langle V_{2, \text { Spin }, c c}^{(1)}(r)\right\rangle=\frac{1}{m^{2}}\left[\left(\alpha_{s}\left\langle\frac{1}{r^{3}}\right\rangle-\frac{\left(b_{c \bar{c}} / 2\right)}{2}\left\langle\frac{1}{r}\right\rangle\right)\langle\mathbf{L} \cdot \mathbf{S}\rangle+\frac{\alpha_{s}}{6}\left\langle\frac{1}{r^{3}}\right\rangle\left\langle\mathbf{S}_{\mathbf{1 2}}\right\rangle\right]
$$

We will adopt the parameter set we obtained fitting this model with $13 c \bar{c}$ as input. Remember that with this model we got a very satisfying agreement between predictions and 
experimental data for the whole spectrum.

In Tables 5.5 and 5.6 we present the results for the diquark wavefunctions and masses, respectively, using this model 2 .

Table 5.5: Results for diquark cc wavefunctions with model 2. Parameters from charmonium in Sec. 4.2.1: $m_{c}=1.4622 \mathrm{GeV}, \alpha_{s}=0.5202, b=b_{c \bar{c}} / 2=$ $0.1463 / 2 \mathrm{GeV}^{2}, \sigma=1.0831 \mathrm{GeV}$.

\begin{tabular}{crrrr}
\hline \hline$N^{2 S+1} \ell$ & $M^{(0)}[\mathrm{GeV}]$ & $|R(0)|^{2}\left[\mathrm{GeV}^{3}\right]$ & $\left\langle r^{2}\right\rangle^{1 / 2}[\mathrm{fm}]$ & $\left\langle\frac{v^{2}}{c^{2}}\right\rangle$ \\
\hline $\mathbf{1}^{\mathbf{3}} \mathbf{S}$ & $\mathbf{3 . 1 3 3 4}$ & $\mathbf{0 . 3 2 9 6}$ & $\mathbf{0 . 5 9 3}$ & $\mathbf{0 . 1 2 3}$ \\
\hline $1^{1} P$ & 3.3530 & 0 & 0.906 & 0.141 \\
\hline $2^{3} S$ & 3.4560 & 0.2370 & 1.147 & 0.167 \\
\hline $2^{1} P$ & 3.6062 & 0 & 1.395 & 0.190 \\
\hline \hline
\end{tabular}

Table 5.6: Results for diquark cc masses with model 2. Parameters from charmonium in Sec. 4.2.1: $m_{c}=1.4622 \mathrm{GeV}, \alpha_{s}=0.5202, b=b_{c \bar{c}} / 2=$ $0.1463 / 2 \mathrm{GeV}^{2}, \sigma=1.0831 \mathrm{GeV}$.

\begin{tabular}{rrrrrrrrrr}
\hline \hline$N^{2 S+1} \ell_{J}$ & $\langle T\rangle$ & $\left\langle V_{V}^{(0)}\right\rangle$ & $\left\langle V_{S}^{(0)}\right\rangle$ & $\left\langle V_{S S}^{(0)}\right\rangle$ & $E^{(0)}$ & $M^{(0)}[\mathrm{MeV}]$ & $\left\langle V_{L S}^{(1)}\right\rangle$ & $\left\langle V_{T}^{(1)}\right\rangle$ & $M_{2}^{f}[\mathrm{MeV}]$ \\
\hline $\mathbf{1}^{\mathbf{3}} \mathbf{S}_{\mathbf{1}}$ & $\mathbf{1 8 0 . 4}$ & $-\mathbf{1 7 3 . 9}$ & $\mathbf{1 9 7 . 9}$ & $\mathbf{4 . 7}$ & $\mathbf{2 0 9 . 0}$ & $\mathbf{3 1 3 3 . 4}$ & $\mathbf{0}$ & $\mathbf{0}$ & $\mathbf{3 1 3 3 . 4}$ \\
\hline $1^{1} P_{1}$ & 206.7 & -93.3 & 316.2 & -0.9 & 428.7 & 3353.0 & 0 & 0 & 3353.0 \\
\hline $2^{3} S_{1}$ & 244.8 & -105.7 & 389.8 & 2.9 & 531.7 & 3456.0 & 0 & 0 & 3456.0 \\
\hline $2^{1} P_{1}$ & 277.5 & -72.3 & 477.9 & -1.2 & 681.9 & 3606.2 & 0 & 0 & 3606.2 \\
\hline \hline
\end{tabular}

Notice that the ground state diquark $1^{3} S_{1}$ has a mass about $35 \mathrm{MeV}$ higher than the $J / \psi$.

\subsection{Comparisons with the Literature}

In the Table 5.7 we show a few results of other works about $c c$ diquarks to be compared with our results in the previous sections. Due to differences in the models and presentation in each reference, we show only the information that can be compared to our results. In particular, we select only the ones that correspond to the (attractive) antitriplet-color configuration. Some models also consider the (repulsive) sextet color configuration.

As we can see clearly, the $1 S$ diquark is very similar in all the models, with mass around $3 \mathrm{GeV}$. 
Table 5.7: Results for diquark cc from literature.

\begin{tabular}{ccccc}
\hline \hline$N \ell$ & $M_{c c}[\mathrm{GeV}]$ & $|R(0)|^{2}\left[\mathrm{GeV}^{3}\right]$ & $\left\langle r^{2}\right\rangle^{1 / 2}[\mathrm{fm}]$ & Ref. \\
\hline $\mathbf{1 S}$ & $\mathbf{3 . 1 3}$ & $(\mathbf{0 . 5 2 3})^{\mathbf{2}}=\mathbf{0 . 2 7 3 5}$ & $\mathbf{0 . 5 8}$ & {$[82]$} \\
$2 S$ & 3.47 & $(0.424)^{2}=0.1798$ & 1.12 & {$[82]$} \\
$2 P$ & 3.35 & - & 0.88 & {$[82]$} \\
\hline $\mathbf{1 S}$ & $\mathbf{3 . 2 2 6}$ & - & - & {$[84]$} \\
\hline $\mathbf{1 S}$ & $\mathbf{3 . 0 6 7}$ & - & - & {$[4]$ mod. I } \\
$\mathbf{1 S}$ & $\mathbf{3 . 0 8 2}$ & - & - & {$[4]$ mod. II } \\
$1 P$ & 3.523 & - & - & {$[4]$ mod. I } \\
$1 P$ & 3.513 & - & - & {$[4]$ mod. II } \\
\hline \hline
\end{tabular}

In order to compare our own results, in Tables 5.8 and 5.9 we present the results for the diquark $1^{3} S_{1}$ used as the building blocks of the tetraquark, with model 1 , model 1 with modified spin-orbit and model 2.

Notice that even though there are differences in the models and respective parameters, the results for the $1^{3} S_{1}$ diquarks are very similar.

Table 5.8: Results for cc $1^{3} S_{1}$ diquark wavefunctions with model 1, model 1 with modified spin-orbit and model 2. Only in model 2 the wavefunction depend on the spin.

\begin{tabular}{ccccccccc}
\hline \hline Model & $m_{c}[\mathrm{GeV}]$ & $\alpha_{s}$ & $b_{c \bar{c}} / 2\left[\mathrm{GeV}^{2}\right]$ & $\sigma[\mathrm{GeV}]$ & $M^{(0)}[\mathrm{GeV}]$ & $|R(0)|^{2}\left[\mathrm{GeV}^{3}\right]$ & $\left\langle r^{2}\right\rangle^{1 / 2}[\mathrm{fm}]$ & $\left\langle\frac{v^{2}}{c^{2}}\right\rangle$ \\
\hline 1 & 1.4155 & 0.4116 & $0.1517 / 2$ & - & $\mathbf{3 . 0 8 4 2}$ & $\mathbf{0 . 2 6 2 4}$ & $\mathbf{0 . 6 1 3}$ & $\mathbf{0 . 1 2 2}$ \\
\hline 1 LS & 1.2819 & 0.3289 & $0.2150 / 2$ & - & $\mathbf{2 . 9 4 9 8}$ & $\mathbf{0 . 2 5 1 5}$ & $\mathbf{0 . 5 9 4}$ & $\mathbf{0 . 1 5 6}$ \\
\hline 2 & 1.4622 & 0.5202 & $0.1463 / 2$ & 1.0831 & $\mathbf{3 . 1 3 3 4}$ & $\mathbf{0 . 3 2 9 6}$ & $\mathbf{0 . 5 9 3}$ & $\mathbf{0 . 1 2 3}$ \\
\hline \hline
\end{tabular}

Table 5.9: Results for $c c 1^{3} S_{1}$ diquark masses with model 1, model 1 with modified spin-orbit and model 2.

\begin{tabular}{ccrrrrrrrr}
\hline \hline Model & $\langle T\rangle$ & $\left\langle V_{V}^{(0)}\right\rangle$ & $\left\langle V_{S}^{(0)}\right\rangle$ & $E^{(0)}$ & $M^{(0)}[\mathrm{MeV}]$ & $\left\langle V_{S S}^{(1)}\right\rangle$ & $\left\langle V_{L S}^{(1)}\right\rangle$ & $\left\langle V_{T}^{(1)}\right\rangle$ & $M_{1}^{f}[\mathrm{MeV}]$ \\
\hline 1 & $\mathbf{1 7 2 . 4}$ & $\mathbf{- 1 3 2 . 0}$ & $\mathbf{2 1 2 . 9}$ & $\mathbf{2 5 3 . 3}$ & $\mathbf{3 0 8 4 . 2}$ & $\mathbf{6 . 0}$ & $\mathbf{0}$ & $\mathbf{0}$ & $\mathbf{3 0 9 0 . 2}$ \\
\hline 1 LS & $\mathbf{2 0 0 . 2}$ & $\mathbf{- 1 0 7 . 3}$ & $\mathbf{2 9 3 . 1}$ & $\mathbf{3 8 6 . 0}$ & $\mathbf{2 9 4 9 . 8}$ & $\mathbf{5 . 6}$ & $\mathbf{0}$ & $\mathbf{0}$ & $\mathbf{2 9 5 5 . 4}$ \\
\hline \hline Model & $\langle T\rangle$ & $\left\langle V_{V}^{(0)}\right\rangle$ & $\left\langle V_{S}^{(0)}\right\rangle$ & $\left\langle V_{S S}^{(0)}\right\rangle$ & $E^{(0)}$ & $M^{(0)}[\mathrm{MeV}]$ & $\left\langle V_{L S}^{(1)}\right\rangle$ & $\left\langle V_{T}^{(1)}\right\rangle$ & $M_{2}^{f}[\mathrm{MeV}]$ \\
\hline 2 & $\mathbf{1 8 0 . 4}$ & $\mathbf{- 1 7 3 . 9}$ & $\mathbf{1 9 7 . 9}$ & $\mathbf{4 . 7}$ & $\mathbf{2 0 9 . 0}$ & $\mathbf{3 1 3 3 . 4}$ & $\mathbf{0}$ & $\mathbf{0}$ & $\mathbf{3 1 3 3 . 4}$ \\
\hline \hline
\end{tabular}




\section{Chapter 6}

\section{The All-Charm Tetraquark}

In this chapter we will consider the diquark-antidiquark tetraquark, treated as a 2-body problem. We calculate the $T_{4 c}$ tetraquark $[c c][\overline{c c}]$, considering the diquarks as point-like structures, with the masses obtained in Chapter 5. For each of the three sets which we used for the diquarks, we now use the same set with the charmonium meson potential, but instead of the charm quark mass, we use the diquark mass $m_{c c}$ as input.

There is one big difference besides the constituents masses: the spins. In mesons and diquarks we had two spin $1 / 2$ particles interacting. Now, since we will consider diquarks in the lowest level of antitriplet color state $\left(1^{3} S_{1}\right)$, the tetraquark will be described with the coupling of two spin 1 particles, so the spin-dependent interactions have more possibilities and different results. We extensively discussed that in Chapter 3, in particular the lengthy calculation of the tensor factor in P-wave states, a theme that is sometimes avoided by the tetraquark works in literature, which tend to consider only ground states where this interaction vanish.

One of the main characteristics of diquark-antidiquark tetraquarks, is their compactness. Also, in contrast with meson molecules, they offer the possibility of radial and orbital excitations.

Based on all the theory developed until here, we finally present the spectrum of the all-charm tetraquark considering the ground states $1 S$, the first orbital excitations $1 P$ (relative to the diquark-antidiquark system), with all the possible combinations of total spin $S=0,1,2$ and total angular momentum $J$ (as discussed in Chapter 3), and both radial excitations $2 S$ and $2 P$, in a total of $20 T_{4 c}$ states built with antitriplet color state with spin 1 cc diquark and antidiquark. These 20 pure states were built considering the coupling of the total spin of the tetraquark $S_{T}$ (composed by the coupling of the total spins of the diquark $S_{d}$ and antidiquark $S_{\bar{d}}$ ) with the relative orbital angular momentum $L_{T}$ between diquark and antidiquark, resulting in a total angular momentum $J_{T}$ of the tetraquark. The corresponding parity and charge-conjugation quantum numbers of each combination can be found in Table 2.2.

\subsection{Model 1}

Just like presented for diquarks, we first used the complete model 1 with parameters obtained from charmonium in a fit with $13 c \bar{c}$ states as input.

In Table 6.1 and 6.2 we present the results for the $T_{4 c}$ wavefunctions and masses, respectively, and in Figure 6.1 we show the mass spectrum including the thresholds for spontaneous decay into pairs of $\eta_{c} \eta_{c}$ or $J / \psi J / \psi$. 
All-Charm Tetraquark Mass Spectrum with Model 1

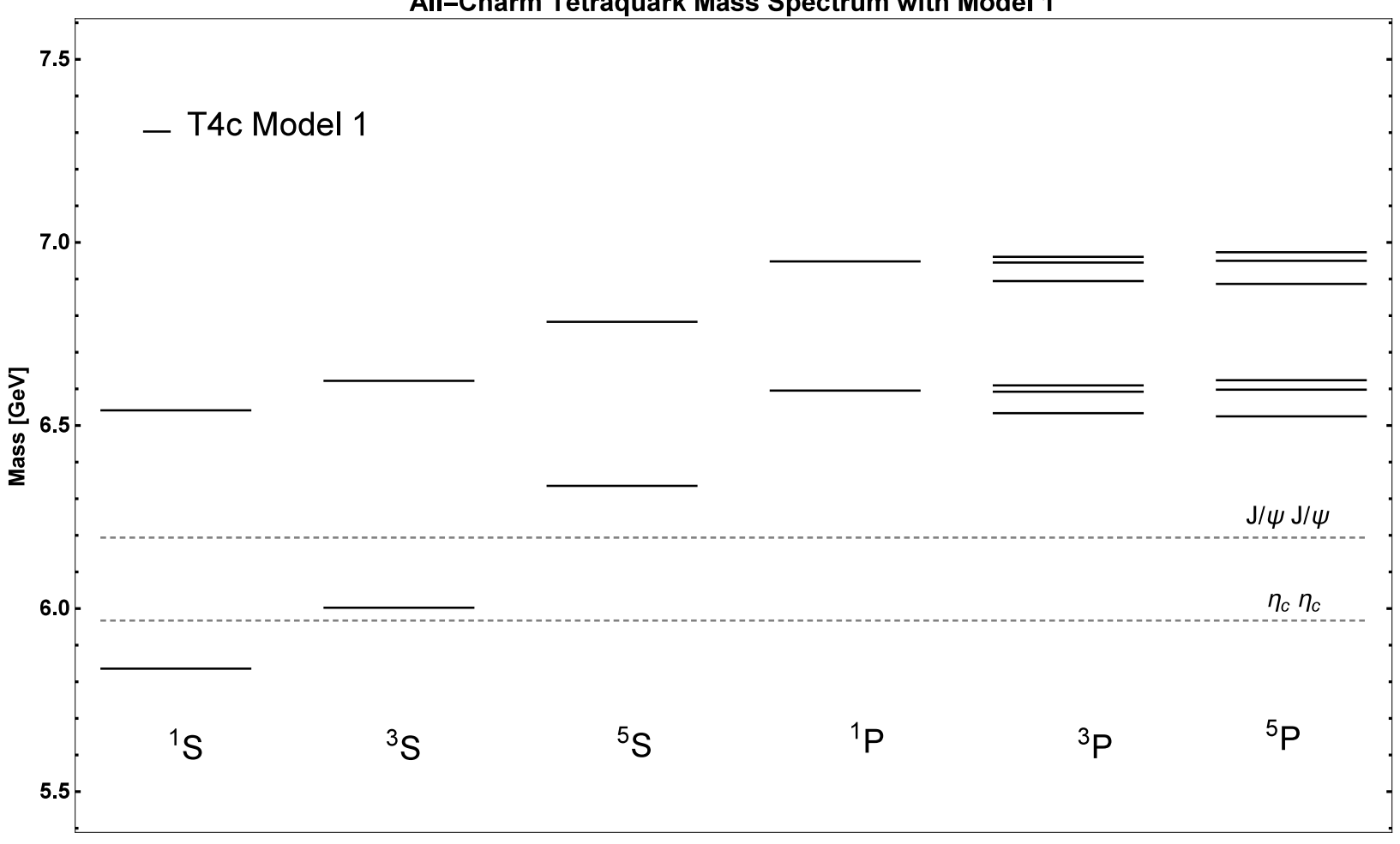

Figure 6.1: Spectrum of $T_{4 c}$ obtained with model 1 and ground state $\left(1^{3} S\right)$ diquark and antidiquark. Parameters are $m_{c c}=3.0902 \mathrm{GeV}, \alpha_{s}=0.4116$ and $b=0.1517 \mathrm{GeV}^{2}$.

Table 6.1: Results for $T_{4 c}$ wavefunctions obtained with model 1 and ground state $\left(1^{3} S\right)$ diquark and antidiquark. Parameters are $m_{c c}=3.0902 \mathrm{GeV}, \alpha_{s}=$ 0.4116 and $b=0.1517 \mathrm{GeV}^{2}$.

\begin{tabular}{lrrrr}
\hline \hline$N \ell$ & $M^{(0)}[\mathrm{GeV}]$ & $|R(0)|^{2}\left[\mathrm{GeV}^{3}\right]$ & $\left\langle r^{2}\right\rangle^{1 / 2}[\mathrm{fm}]$ & $\left\langle\frac{v^{2}}{c^{2}}\right\rangle$ \\
\hline $1 S$ & 6.1687 & 4.3437 & 0.285 & 0.130 \\
\hline $1 P$ & 6.5954 & 0 & 0.507 & 0.102 \\
\hline $2 S$ & 6.7026 & 2.1029 & 0.634 & 0.122 \\
\hline $2 P$ & 6.9481 & 0 & 0.813 & 0.125 \\
\hline \hline
\end{tabular}

Notice how small is the tetraquark. The $1 S$ has a root mean square radius about 0.3 $\mathrm{fm}$, and even the $2 P$, with one radial and one orbital excitation is smaller than $1 \mathrm{fm}$. The mean square velocities of the diquarks inside the tetraquark (within this approximation of 2-body system) are also very small, close to 0.1 of $c^{2}$, smaller than the charm quark inside the charmonium mesons, which is probably due to the fact that the diquark mass is about twice the charm quark mass.

One interesting point is how huge is the value of the wavefunction at the origin, and therefore also the spin-spin interaction. It is even comparable to the zeroth-order contributions of the potential. Another reason for its huge value is that the factor $\left\langle\mathbf{S}_{\mathbf{1}} \cdot \mathbf{S}_{\mathbf{2}}\right\rangle$ 
is much higher for the interaction of two spin 1 particles than in the case of two spin $1 / 2$ particles (see Section 3.1). One could question if the perturbative treatment is still acceptable.

The contribution of the vector (Coulomb) term due to one gluon exchange is also very big, being predominant for the S-wave states. One modification that would make both the spin-spin and Coulomb contribution smaller would be to reduce the value of the coupling constant $\alpha_{s}$, since both interactions are proportional to it. Actually, it is expected to decrease as the energy scale increase, so rescaling it to the tetraquark level could be an appropriate correction. It is also curious that the energy eigenvalue is small but negative for the $1 S$ states.

Another interesting fact is that almost all the $T_{4 c}$ states are above the thresholds for spontaneous decays in charmonium meson pairs (around $5.97 \mathrm{GeV}$ for an $\eta_{c}$ pair and 6.19 $\mathrm{GeV}$ for a $J / \psi$ pair), indicating that these could be appropriate channels to search for these states in experimental measures.

Table 6.2: Results for $T_{4 c}$ masses obtained with model 1 and ground state and $\left(1^{3} S\right)$ diquark and antidiquark. Parameters are $m_{c c}=3.0902$ GeV, $\alpha_{s}=$ 0.4116 and $b=0.1517 \mathrm{GeV}^{2}$.

\begin{tabular}{crrrrrrrrrr}
\hline \hline$N^{2 S+1} \ell_{J}$ & $\langle T\rangle$ & $\left\langle V_{V}^{(0)}\right\rangle$ & $\left\langle V_{S}^{(0)}\right\rangle$ & $E^{(0)}$ & $M^{(0)}[\mathrm{MeV}]$ & $\left\langle V_{S S}^{(1)}\right\rangle$ & $\left\langle V_{L S}^{(1)}\right\rangle$ & $\left\langle V_{T}^{(1)}\right\rangle$ & $M_{1}^{f}[\mathrm{MeV}]$ & $J^{P C}$ \\
\hline $1^{1} S_{0}$ & 400.9 & -607.2 & 194.6 & -11.8 & 6168.7 & -333.0 & 0 & 0 & 5835.9 & $0^{++}$ \\
$1^{3} S_{1}$ & 400.9 & -607.2 & 194.6 & -11.8 & 6168.7 & -166.0 & 0 & 0 & 6002.3 & $1^{+-}$ \\
$1^{5} S_{2}$ & 400.9 & -607.2 & 194.6 & -11.8 & 6168.7 & 166.0 & 0 & 0 & 6335.2 & $2^{++}$ \\
\hline $1^{1} P_{1}$ & 316.6 & -267.4 & 365.8 & 414.9 & 6595.4 & 0 & 0 & 0 & 6595.4 & $1^{--}$ \\
\hline $1^{3} P_{0}$ & 316.6 & -267.4 & 365.8 & 414.9 & 6595.4 & 0 & -33.9 & -27.8 & 6533.6 & $0^{-+}$ \\
$1^{3} P_{1}$ & 316.6 & -267.4 & 365.8 & 414.9 & 6595.4 & 0 & -17.0 & 13.9 & 6592.3 & $1^{-+}$ \\
$1^{3} P_{2}$ & 316.6 & -267.4 & 365.8 & 414.9 & 6595.4 & 0 & 17.0 & -2.8 & 6609.6 & $2^{-+}$ \\
\hline $1^{5} P_{1}$ & 316.6 & -267.4 & 365.8 & 414.9 & 6595.4 & 0 & -50.9 & -19.5 & 6525.0 & $1^{--}$ \\
$1^{5} P_{2}$ & 316.6 & -267.4 & 365.8 & 414.9 & 6595.4 & 0 & -17.0 & 19.5 & 6597.8 & $2^{--}$ \\
$1^{5} P_{3}$ & 316.6 & -267.4 & 365.8 & 414.9 & 6595.4 & 0 & 33.9 & -5.6 & 6623.7 & $3^{--}$ \\
\hline $2^{1} S_{0}$ & 376.3 & -303.4 & 449.2 & 522.1 & 6702.6 & -161.0 & 0 & 0 & 6541.4 & $0^{++}$ \\
$2^{3} S_{1}$ & 376.3 & -303.4 & 449.2 & 522.1 & 6702.6 & -80.6 & 0 & 0 & 6622.0 & $1^{+-}$ \\
$2^{5} S_{2}$ & 376.3 & -303.4 & 449.2 & 522.1 & 6702.6 & 80.6 & 0 & 0 & 6783.1 & $2^{++}$ \\
\hline $2^{1} P_{1}$ & 387.8 & -197.9 & 577.8 & 767.7 & 6948.1 & 0 & 0 & 0 & 6948.1 & $1^{--}$ \\
\hline $2^{3} P_{0}$ & 387.8 & -197.9 & 577.8 & 767.7 & 6948.1 & 0 & -29.9 & -23.7 & 6894.5 & $0^{-+}$ \\
$2^{3} P_{1}$ & 387.8 & -197.9 & 577.8 & 767.7 & 6948.1 & 0 & -14.9 & 11.9 & 6945.1 & $1^{-+}$ \\
$2^{3} P_{2}$ & 387.8 & -197.9 & 577.8 & 767.7 & 6948.1 & 0 & 14.9 & -2.4 & 6960.7 & $2^{-+}$ \\
\hline $2^{5} P_{1}$ & 387.8 & -197.9 & 577.8 & 767.7 & 6948.1 & 0 & -44.8 & -16.7 & 6886.7 & $1^{--}$ \\
$2^{5} P_{2}$ & 387.8 & -197.9 & 577.8 & 767.7 & 6948.1 & 0 & -14.9 & 16.6 & 6949.8 & $2^{--}$ \\
$2^{5} P_{3}$ & 387.8 & -197.9 & 577.8 & 767.7 & 6948.1 & 0 & 29.9 & -4.7 & 6973.3 & $3^{--}$ \\
\hline \hline
\end{tabular}




\subsection{Model 1 with modified Spin-Orbit}

Here we present the results for the $T_{4 c}$ using model 1 with the second term of the spin-orbit interaction removed. The parameters are the ones obtained with the fit of the charmonium spectrum (the 6 input states $1 S$ and $1 P$ ), and the respective diquark mass obtained with the same model and parameter set.

In Table 6.3 and 6.4 we present the results for the $T_{4 c}$ wavefunctions and masses, respectively, and in Figure 6.2 we show the mass spectrum.

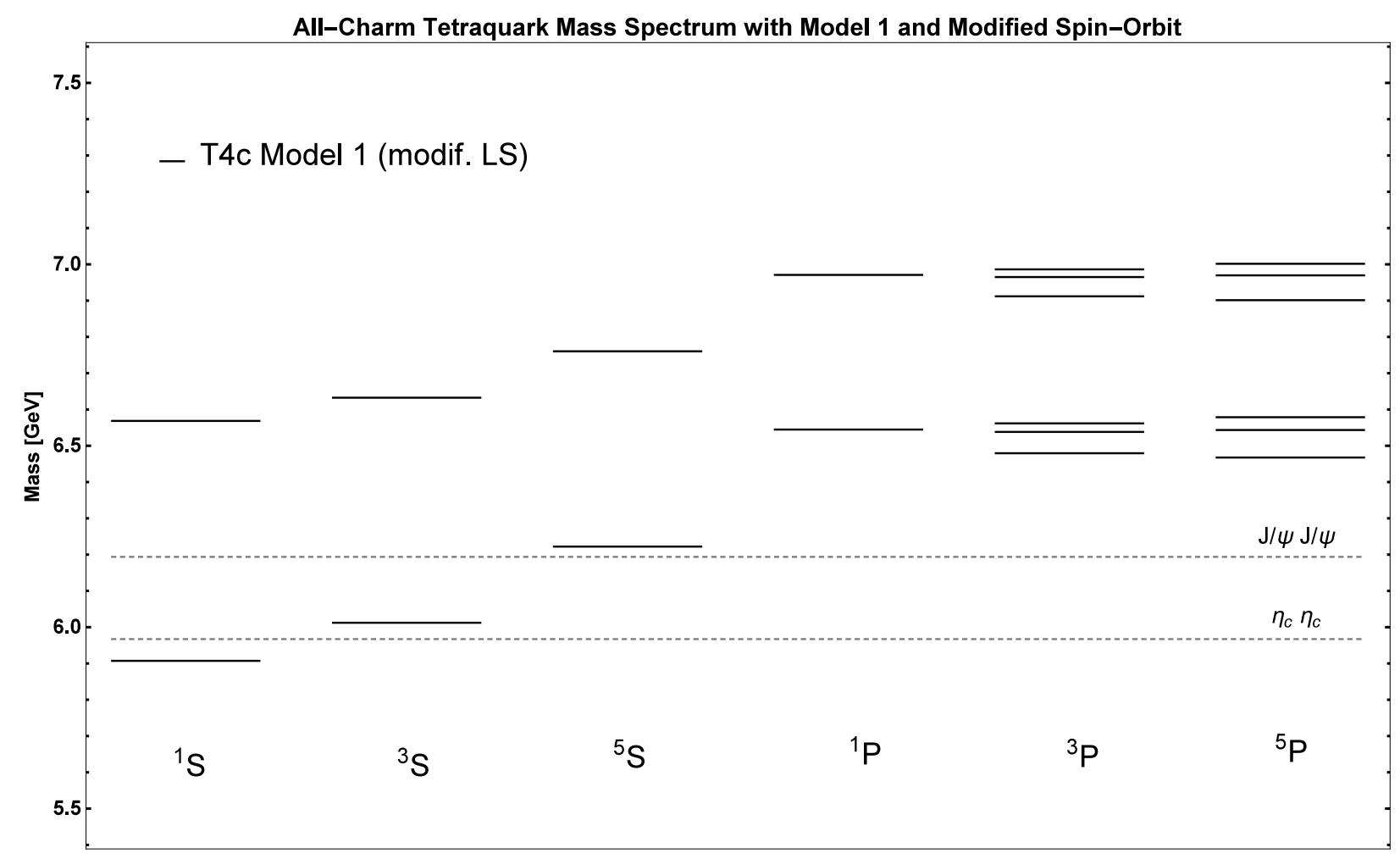

Figure 6.2: Spectrum of $T_{4 c}$ obtained with model 1 with modified spin-orbit and ground state $\left(1^{3} S\right)$ diquark and antidiquark. Parameters are $m_{c c}=2.9554$ $\mathrm{GeV}, \alpha_{s}=0.3289, b=0.2150 \mathrm{GeV}^{2}$.

Table 6.3: Results for $T_{4 c}$ wavefunctions obtained with model 1 with modified spin-orbit and ground state $\left(1^{3} S\right)$ diquark and antidiquark. Parameters are $m_{c c}=2.9554 \mathrm{GeV}, \alpha_{s}=0.3289, b=0.2150 \mathrm{GeV}^{2}$.

\begin{tabular}{lrrrr}
\hline \hline$N \ell$ & $M^{(0)}[\mathrm{GeV}]$ & $|R(0)|^{2}\left[\mathrm{GeV}^{3}\right]$ & $\left\langle r^{2}\right\rangle^{1 / 2}[\mathrm{fm}]$ & $\left\langle\frac{v^{2}}{c^{2}}\right\rangle$ \\
\hline $1 S$ & 6.1172 & 3.1355 & 0.296 & 0.126 \\
\hline $1 P$ & 6.5447 & 0 & 0.485 & 0.121 \\
\hline $2 S$ & 6.6964 & 1.9106 & 0.608 & 0.146 \\
\hline $2 P$ & 6.9708 & 0 & 0.757 & 0.158 \\
\hline \hline
\end{tabular}


Notice that the results are very similar to the ones in the previous section. The only changes are analogous to the ones that happened in charmonium: the P-wave splitting is a bit wider, while the spin-spin correction is smaller (and also the contribution of the Coulomb term), what was expected since the strong coupling constant is smaller in this set. The energy eigenvalue of the $1 S$ states is positive and large now, in contrast with the results of the previous section.

It is interesting that the wavefunction properties and the range of masses of the $T_{4 c}$ aren't so different from the ones in the previous section. The lowest state is also below $6 \mathrm{GeV}$, and all the others are within the range $6-7 \mathrm{GeV}$ too. Again, the lowest state is below the $\eta_{c}$ pair threshold and the second-lowest one is above this threshold but below the one for decay in a $J / \psi$ pair.

Table 6.4: Results for $T_{4 c}$ masses obtained with model 1 with modified spinorbit and ground state $\left(1^{3} S\right)$ diquark and antidiquark. Parameters are $m_{c c}=$ $2.9554 \mathrm{GeV}, \alpha_{s}=0.3289, b=0.2150 \mathrm{GeV}^{2}$.

\begin{tabular}{crrrrrrrrrr}
\hline \hline$N^{2 S+1} \ell_{J}$ & $\langle T\rangle$ & $\left\langle V_{V}^{(0)}\right\rangle$ & $\left\langle V_{S}^{(0)}\right\rangle$ & $E^{(0)}$ & $M^{(0)}[\mathrm{MeV}]$ & $\left\langle V_{S S}^{(1)}\right\rangle$ & $\left\langle V_{L S}^{(1)}\right\rangle$ & $\left\langle V_{T}^{(1)}\right\rangle$ & $M_{1}^{f}[\mathrm{MeV}]$ & $J^{P C}$ \\
\hline $1^{1} S_{0}$ & 370.9 & -453.2 & 288.7 & 206.4 & 6117.2 & -210.0 & 0 & 0 & 5907.3 & $0^{++}$ \\
$1^{3} S_{1}$ & 370.9 & -453.2 & 288.7 & 206.4 & 6117.2 & -105.0 & 0 & 0 & 6012.2 & $1^{+-}$ \\
$1^{5} S_{2}$ & 370.9 & -453.2 & 288.7 & 206.4 & 6117.2 & 105.0 & 0 & 0 & 6222.1 & $2^{++}$ \\
\hline $1^{1} P_{1}$ & 359.0 & -221.5 & 496.5 & 633.9 & 6544.7 & 0 & 0 & 0 & 6544.7 & $1^{--}$ \\
\hline $1^{3} P_{0}$ & 359.0 & -221.5 & 496.5 & 633.9 & 6544.7 & 0 & -39.2 & -26.1 & 6479.4 & $0^{-+}$ \\
$1^{3} P_{1}$ & 359.0 & -221.5 & 496.5 & 633.9 & 6544.7 & 0 & -19.6 & 13.1 & 6538.2 & $1^{-+}$ \\
$1^{3} P_{2}$ & 359.0 & -221.5 & 496.5 & 633.9 & 6544.7 & 0 & 19.6 & -2.6 & 6561.7 & $2^{-+}$ \\
\hline $1^{5} P_{1}$ & 359.0 & -221.5 & 496.5 & 633.9 & 6544.7 & 0 & -58.8 & -18.3 & 6467.6 & $1^{--}$ \\
$1^{5} P_{2}$ & 359.0 & -221.5 & 496.5 & 633.9 & 6544.7 & 0 & -19.6 & 18.3 & 6543.4 & $2^{--}$ \\
$1^{5} P_{3}$ & 359.0 & -221.5 & 496.5 & 633.9 & 6544.7 & 0 & 39.2 & -5.2 & 6578.7 & $3^{--}$ \\
\hline $2^{1} S_{0}$ & 431.1 & -253.8 & 608.3 & 785.6 & 6696.4 & -128.0 & 0 & 0 & 6568.5 & $0^{++}$ \\
$2^{3} S_{1}$ & 431.1 & -253.8 & 608.3 & 785.6 & 6696.4 & -64.0 & 0 & 0 & 6632.4 & $1^{+-}$ \\
$2^{5} S_{2}$ & 431.1 & -253.8 & 608.3 & 785.6 & 6696.4 & 64.0 & 0 & 0 & 6760.4 & $2^{++}$ \\
\hline $2^{1} P_{1}$ & 465.7 & -168.5 & 762.8 & 1060.1 & 6970.8 & 0 & 0 & 0 & 6970.8 & $1^{--}$ \\
\hline $2^{3} P_{0}$ & 465.7 & -168.5 & 762.8 & 1060.1 & 6970.8 & 0 & -35.4 & -23.6 & 6911.9 & $0^{-+}$ \\
$2^{3} P_{1}$ & 465.7 & -168.5 & 762.8 & 1060.1 & 6970.8 & 0 & -17.7 & 11.8 & 6965.0 & $1^{-+}$ \\
$2^{3} P_{2}$ & 465.7 & -168.5 & 762.8 & 1060.1 & 6970.8 & 0 & 17.7 & -2.4 & 6986.2 & $2^{-+}$ \\
\hline $2^{5} P_{1}$ & 465.7 & -168.5 & 762.8 & 1060.1 & 6970.8 & 0 & -53.1 & -16.5 & 6901.2 & $1--$ \\
$2^{5} P_{2}$ & 465.7 & -168.5 & 762.8 & 1060.1 & 6970.8 & 0 & -17.7 & 16.5 & 6969.7 & $2^{--}$ \\
$2^{5} P_{3}$ & 465.7 & -168.5 & 762.8 & 1060.1 & 6970.8 & 0 & 35.4 & -4.7 & 7001.5 & $3^{--}$ \\
\hline \hline
\end{tabular}




\section{3 $\quad$ Model 2}

In this model 2 the spin-spin interaction is treated non-perturbatively. In mesons and diquarks we had only two possibilities of total spin when combining two spin $1 / 2$ particles $S=0,1$. Now, since we consider spin 1 diquark and antidiquark, we have three possibilities of total spin $S=0,1,2$, and therefore three different zeroth-order potentials (and consequently three wavefunctions for each $N \ell$ state):

$$
\begin{array}{ll}
V_{2, T 4 c}^{(0)}(r)=-\frac{4}{3} \frac{\alpha_{s}}{r}+b r-\frac{64 \pi \alpha_{s}}{9 m^{2}}\left(\frac{\sigma}{\sqrt{\pi}}\right)^{3} \mathrm{e}^{-\sigma^{2} r^{2}}, & (S=0) \\
V_{2, T 4 c}^{(0)}(r)=-\frac{4}{3} \frac{\alpha_{s}}{r}+b r-\frac{32 \pi \alpha_{s}}{9 m^{2}}\left(\frac{\sigma}{\sqrt{\pi}}\right)^{3} \mathrm{e}^{-\sigma^{2} r^{2}}, \quad(S=1) \\
V_{2, T 4 c}^{(0)}(r)=-\frac{4}{3} \frac{\alpha_{s}}{r}+b r+\frac{32 \pi \alpha_{s}}{9 m^{2}}\left(\frac{\sigma}{\sqrt{\pi}}\right)^{3} \mathrm{e}^{-\sigma^{2} r^{2}}, \quad(S=2)
\end{array}
$$

We use the set of parameters and diquark mass obtained using the fit of the charmonium spectrum with 13 input and model 2. In Tables 6.5 and 6.6 we present the wavefunction and masses of the $T_{4 c}$, respectively, and in Figure 6.3 the mass spectrum.

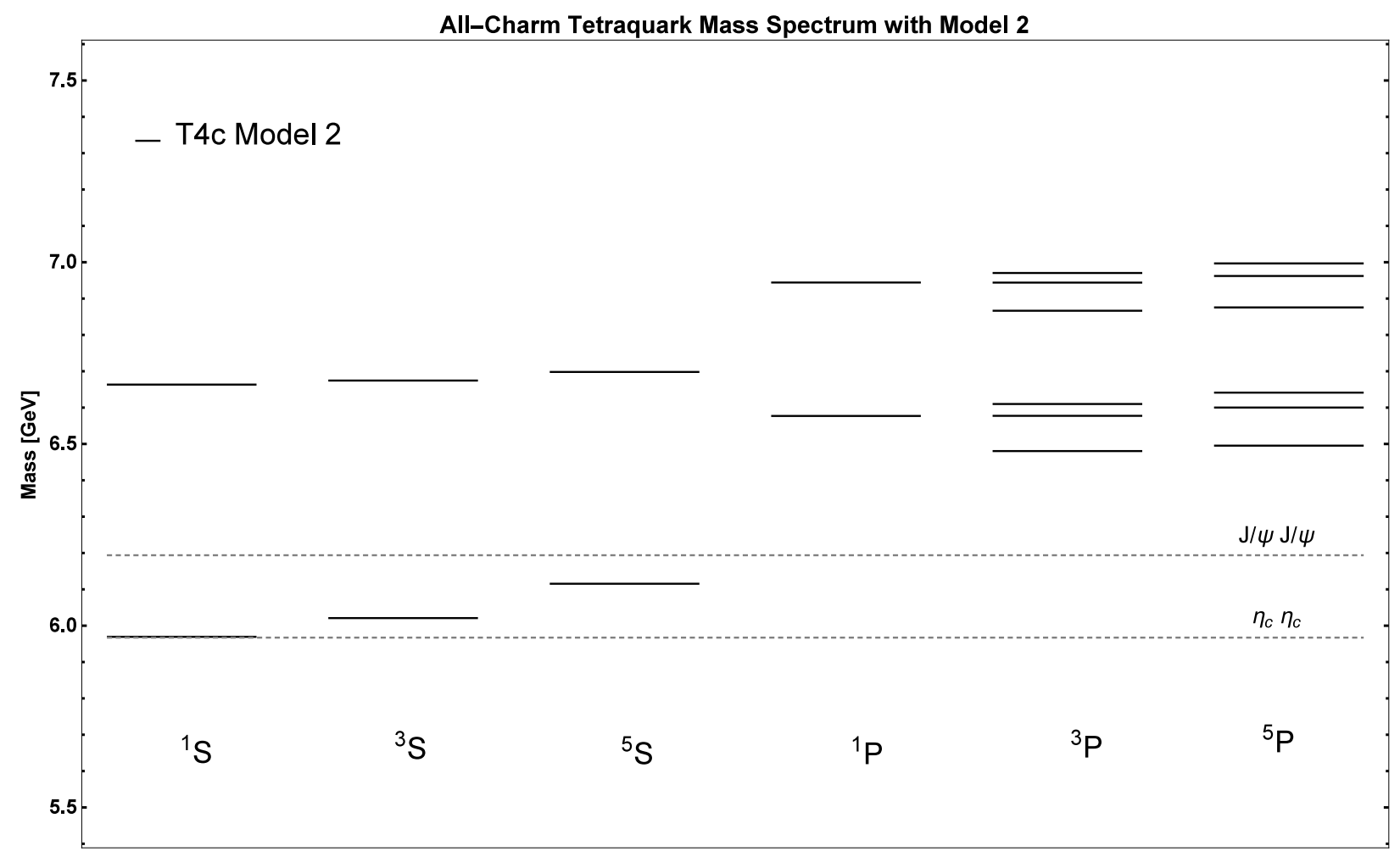

Figure 6.3: Spectrum of $T_{4 c}$ obtained with model 2 and ground state $\left(1^{3} S\right.$ ) diquark and antidiquark. Parameters are $m_{c c}=3133.4 \mathrm{GeV}, \alpha_{s}=0.5202$, $b=0.1463 \mathrm{GeV}^{2}, \sigma=1.0831 \mathrm{GeV}$. 
Table 6.5: Results for $T_{4 c}$ wavefunctions with model 2 and ground state $\left(1^{3} S\right)$ diquark and antidiquark. Parameters are $m_{c c}=3133.4 \mathrm{GeV}, \alpha_{s}=0.5202$, $b=0.1463 \mathrm{GeV}^{2}, \sigma=1.0831 \mathrm{GeV}$.

\begin{tabular}{crrrr}
\hline \hline$N^{2 S+1} \ell$ & $M^{(0)}[\mathrm{GeV}]$ & $|R(0)|^{2}\left[\mathrm{GeV}^{3}\right]$ & $\left\langle r^{2}\right\rangle^{1 / 2}[\mathrm{fm}]$ & $\left\langle\frac{v^{2}}{c^{2}}\right\rangle$ \\
\hline $1^{1} S$ & 5.9694 & 8.4219 & 0.232 & 0.199 \\
$1^{3} S$ & 6.0209 & 7.8384 & 0.241 & 0.183 \\
$1^{5} S$ & 6.1154 & 6.6727 & 0.264 & 0.153 \\
\hline $1^{1} P$ & 6.5771 & 0 & 0.471 & 0.119 \\
$1^{3} P$ & 6.5847 & 0 & 0.478 & 0.115 \\
$1^{5} P$ & 6.5984 & 0 & 0.491 & 0.107 \\
\hline $2^{1} S$ & 6.6633 & 2.8414 & 0.588 & 0.131 \\
$2^{3} S$ & 6.6745 & 2.8528 & 0.595 & 0.130 \\
$2^{5} S$ & 6.6981 & 2.8616 & 0.610 & 0.129 \\
\hline $2^{1} P$ & 6.9441 & 0 & 0.785 & 0.132 \\
$2^{3} P$ & 6.9500 & 0 & 0.790 & 0.130 \\
$2^{5} P$ & 6.9610 & 0 & 0.800 & 0.126 \\
\hline \hline
\end{tabular}

Now we obtain some very interesting changes. First, the masses of the 20 states are still in the same range between 6 and $7 \mathrm{GeV}$. It is surprising how this pattern holds in the three sets. But now there is a significant change in the $1 S$ states: the lowest one is right on the line of the $\eta_{c}$ pair threshold, and now the other two are within this threshold and the one of $J / \psi$ pair, while in the previous sets, only the second-lowest was below the $J / \psi$ pair threshold.

The most peculiar result is regarding the hyperfine interaction. The wavefunction at the origin of the $1 S$ states is much larger than the ones of model 1. The spin-spin interaction is very curious, because it is smaller than in the results with model 1 , even though the wavefunction at the origin is much larger. The attenuation of the spin-spin interaction seems to be an effect of the gaussian function. Another interesting point is that the Coulomb contribution in the $1 S$ states is predominant over the confinement term, what causes the energy eigenvalue to be large and negative for the three $1 S$ states, indicating that the gluon exchange between diquarks is the dominant binding mechanism. However, we have to take into account that the $\alpha_{s}$ parameter is very large in model 2. Also, it is important to notice that the spin-spin interaction is now part of the energy eigenvalue. 
Table 6.6: Results for $T_{4 c}$ masses with model 2 and ground state $\left(1^{3} S\right)$ diquark and antidiquark. Parameters are $m_{c c}=3133.4 \mathrm{GeV}, \alpha_{s}=0.5202, b=0.1463$ $\mathrm{GeV}^{2}, \sigma=1.0831 \mathrm{GeV}$.

\begin{tabular}{crrrrrrrrrr}
\hline \hline$N^{2 S+1} \ell_{J}$ & $\langle T\rangle$ & $\left\langle V_{V}^{(0)}\right\rangle$ & $\left\langle V_{S}^{(0)}\right\rangle$ & $\left\langle V_{S S}^{(0)}\right\rangle$ & $E^{(0)}$ & $M^{(0)}[\mathrm{MeV}]$ & $\left\langle V_{L S}^{(1)}\right\rangle$ & $\left\langle V_{T}^{(1)}\right\rangle$ & $M_{2}^{f}[\mathrm{MeV}]$ & $J^{P C}$ \\
\hline $1^{1} S_{0}$ & 624.0 & -966.6 & 151.1 & -106.0 & -297.3 & 5969.4 & 0 & 0 & 5969.4 & $0^{++}$ \\
$1^{3} S_{1}$ & 574.8 & -928.0 & 157.6 & -50.2 & -245.8 & 6020.9 & 0 & 0 & 6020.9 & $1^{+-}$ \\
$1^{5} S_{2}$ & 479.4 & -847.5 & 172.5 & 44.3 & -151.3 & 6115.4 & 0 & 0 & 6115.4 & $2^{++}$ \\
\hline $1^{1} P_{1}$ & 372.6 & -371.8 & 325.3 & -15.8 & 310.3 & 6577.1 & 0 & 0 & 6577.1 & $1^{--}$ \\
\hline $1^{3} P_{0}$ & 358.9 & -364.3 & 330.7 & -7.4 & 318.0 & 6584.7 & -59.4 & -44.8 & 6480.4 & $0^{-+}$ \\
$1^{3} P_{1}$ & 358.9 & -364.3 & 330.7 & -7.4 & 318.0 & 6584.7 & -29.7 & 22.4 & 6577.4 & $1^{-+}$ \\
$1^{3} P_{2}$ & 358.9 & -364.3 & 330.7 & -7.4 & 318.0 & 6584.7 & 29.7 & -4.5 & 6609.9 & $2^{-+}$ \\
\hline $1^{5} P_{1}$ & 335.4 & -350.8 & 340.7 & 6.4 & 331.7 & 6598.4 & -75.9 & -27.2 & 6495.4 & $1^{--}$ \\
$1^{5} P_{2}$ & 335.4 & -350.8 & 340.7 & 6.4 & 331.7 & 6598.4 & -25.3 & 27.1 & 6600.2 & $2^{--}$ \\
$1^{5} P_{3}$ & 335.4 & -350.8 & 340.7 & 6.4 & 331.7 & 6598.4 & 50.6 & -7.7 & 6641.2 & $3^{--}$ \\
\hline $2^{1} S_{0}$ & 410.8 & -397.0 & 404.6 & -21.8 & 396.6 & 6663.3 & 0 & 0 & 6663.3 & $0^{++}$ \\
$2^{3} S_{1}$ & 408.7 & -398.2 & 408.7 & -11.4 & 407.8 & 6674.5 & 0 & 0 & 6674.5 & $1^{+-}$ \\
$2^{5} S_{2}$ & 403.0 & -400.7 & 416.8 & 12.3 & 431.4 & 6698.1 & 0 & 0 & 6698.1 & $2^{++}$ \\
\hline $2^{1} P_{1}$ & 414.9 & -262.9 & 537.5 & -12.0 & 677.4 & 6944.1 & 0 & 0 & 6944.1 & $1^{--}$ \\
\hline $2^{3} P_{0}$ & 407.8 & -260.0 & 541.2 & -5.7 & 683.3 & 6950.0 & -47.9 & -35.6 & 6866.5 & $0^{-+}$ \\
$2^{3} P_{1}$ & 407.8 & -260.0 & 541.2 & -5.7 & 683.3 & 6950.0 & -23.9 & 17.8 & 6943.9 & $1^{-+}$ \\
$2^{3} P_{2}$ & 407.8 & -260.0 & 541.2 & -5.7 & 683.3 & 6950.0 & 23.9 & -3.6 & 6970.4 & $2^{-+}$ \\
\hline $2^{5} P_{1}$ & 394.5 & -254.2 & 548.7 & 5.2 & 694.3 & 6961.0 & -63.1 & -22.2 & 6875.6 & $1^{--}$ \\
$2^{5} P_{2}$ & 394.5 & -254.2 & 548.7 & 5.2 & 694.3 & 6961.0 & -21.0 & 22.2 & 6962.1 & $2^{--}$ \\
$2^{5} P_{3}$ & 394.5 & -254.2 & 548.7 & 5.2 & 694.3 & 6961.0 & 42.1 & -6.3 & 6996.7 & $3^{--}$ \\
\hline \hline
\end{tabular}




\subsection{Comparisons with the Literature}

In this section we restrict ourselves to point out a few results of other works that also investigate the existence and properties of this state composed of four charm quarks. A historical overview of the evolution of the literature on this subject can be found in the introduction of our work, in Section 1.3, where these and other works were commented. Some of these references also consider the sextet structure of the diquarks (which can also lead to a color singlet tetraquark). In the following tables we try to compile the essential results of some of them. First, we show the results of Ref. [4] in Tables 6.7 and 6.8.

Table 6.7: Results for the $T_{4 c}$ mass (without spin-corrections) from Ref. [4].

\begin{tabular}{cccc}
\hline \hline$N \ell$ & $M_{T 4 c}^{(0)}[\mathrm{GeV}]$ & Model & Color \\
\hline $1 S$ & 6.437 & I & $\overline{3}-3$ \\
$1 S$ & 6.450 & II & $\overline{3}-3$ \\
$1 S$ & 6.383 & I & $6-\overline{6}$ \\
$1 S$ & 6.400 & II & $6-\overline{6}$ \\
$1 S$ & 6.276 & Bag & $\overline{3}-3$ \\
$1 S$ & 6.252 & Bag & $6-\overline{6}$ \\
\hline $1 P$ & 6.718 & I & $\overline{3}-3$ \\
$1 P$ & 6.714 & II & $\overline{3}-3$ \\
$1 P$ & 6.832 & I & $6-\overline{6}$ \\
$1 P$ & 6.822 & II & $6-\overline{6}$ \\
\hline \hline
\end{tabular}

Table 6.8: Results for the spin shifts of the $T_{4 c}$ from Ref. [4].

\begin{tabular}{cclrccc}
\hline \hline$N \ell$ & $M_{T 4 c}^{(0)}[\mathrm{GeV}]$ & $J^{P(C)}$ & $\mathrm{SS}[\mathrm{GeV}]$ & $\mathrm{LS}+\mathrm{T}[\mathrm{GeV}]$ & Model & Color \\
\hline $1 S$ & 6.383 & $0^{+}$ & 0.017 & - & $\mathrm{I}$ & $6-\overline{6}$ \\
$1 S$ & 6.437 & $0^{+}$ & -0.011 & - & $\mathrm{I}$ & $\overline{3}-3$ \\
$1 S$ & 6.437 & $1^{+}$ & 0.003 & - & $\mathrm{I}$ & $\overline{3}-3$ \\
$1 S$ & 6.437 & $2^{+}$ & 0.032 & - & $\mathrm{I}$ & $\overline{3}-3$ \\
\hline $1 P$ & 6.832 & $1^{--}$ & 0.011 & 0 & $\mathrm{I}$ & $6-\overline{6}$ \\
$1 P$ & 6.718 & $0^{-+}$ & 0.010 & -0.023 & $\mathrm{I}$ & $\overline{3}-3$ \\
$1 P$ & 6.718 & $1^{--}$ & 0.020 & -0.024 & $\mathrm{I}$ & $\overline{3}-3$ \\
\hline \hline
\end{tabular}

In this work a variational method with Gaussian trial wavefunctions was employed to study all-heavy tetraquarks, using a 4-body coordinate system. The interactions were described with potential due to exchange of color octets in 2-body forces. Two potentials were used: the model I is a Cornell-type (Coulomb plus linear) and the model II is of the form $A+B r^{\beta}$. Also a version of the MIT Bag model was used with the Born-Oppenheimer 
approximation. Both color structures were considered, $\overline{3}-3$ and $6-\overline{6}$. S-wave and P-wave were considered with both potentials, and spin shifts were calculated with the Cornell-like potential.

In Table 6.9 we compile the results of Refs. [47, 48], where the $T_{4 c}$ production was studied. The estimates for the $T_{4 c}$ are very similar to our work, where the tetraquark is considered as a diquark-antidiquark in the $\overline{3}-3$ configuration. They used the diquark results of Ref. [82], where the $c c$ diquark was calculated as a baryon constituent (we also compared these diquark results with ours in Section 5.4). In this work the same strategy of dividing the problem in 2body systems is used, but only S-wave are calculated, and the spin-spin splitting is considered between each spin $1 / 2$ constituent pair, using the wavefunction at the origin of the diquark or of the charmonium, depending on the pair interacting.

Table 6.9: Results for the $T_{4 c}$ from Refs. [47, 48].

\begin{tabular}{ccccccc}
\hline \hline$N \ell$ & $M_{T 4 c}^{(0)}[\mathrm{GeV}]$ & $|\Psi(0)|\left[\mathrm{GeV}^{3 / 2}\right]$ & $\langle r\rangle[\mathrm{fm}]$ & $J^{P C}$ & $M_{T 4 c}^{f}[\mathrm{GeV}]$ & Color \\
\hline $1 S$ & 6.12 & 0.47 & 0.29 & $0^{++}$ & 5.97 & $\overline{3}-3$ \\
$1 S$ & 6.12 & 0.47 & 0.29 & $1^{+-}$ & 6.05 & $\overline{3}-3$ \\
$1 S$ & 6.12 & 0.47 & 0.29 & $2^{++}$ & 6.22 & $\overline{3}-3$ \\
\hline \hline
\end{tabular}

In Table 6.10 we now compare our results for the $\mathrm{S}$-wave $T_{4 c}$ with a few of the recent diquark-antidiquark works, the one with antitriplet diquark from 2012 [47, 48], and the two from 2016, the color-magnetic model [50] and the Sum Rules [51]:

Table 6.10: Comparison of our results for the $S$-wave $T_{4 c}$.

\begin{tabular}{ccccccc}
\hline \hline$J^{P C}$ & $M_{1}^{f}[\mathrm{GeV}]$ & $M_{1-L S}^{f}[\mathrm{GeV}]$ & $M_{2}^{f}[\mathrm{GeV}]$ & Ref. [47, 48] & Ref. [50] & Ref. [51] \\
\hline $0^{++}$ & 5.8359 & 5.9073 & 5.9694 & 5.966 & $5.617-6.254$ & $6.44-7.15$ \\
$1^{+-}$ & 6.0023 & 6.0122 & 6.0209 & 6.051 & $5.720-6.137$ & $6.37-6.51$ \\
$2^{++}$ & 6.3352 & 6.2221 & 6.1154 & 6.223 & $5.777-6.194$ & $6.51-6.37$ \\
\hline \hline
\end{tabular}

At last, in Table 6.11 we compare our results for the $\mathrm{P}$-wave $T_{4 c}$ with a the diquarkantidiquark predictions of Chao [32] from 1981, the recent diquark-antidiquark Sum Rules [51] from 2016 and the lattice results [44] from 2006:

Table 6.11: Comparison of our results for the P-wave $T_{4 c}$.

\begin{tabular}{cccccc|cc}
\hline \hline$J^{P C}$ & $N^{2 S+1} \ell_{J}$ & $M_{1}^{f}[\mathrm{GeV}]$ & $M_{1-L S}^{f}[\mathrm{GeV}]$ & $M_{2}^{f}[\mathrm{GeV}]$ & Ref. [32] & Ref. [51] & Ref. [44] \\
\hline $1^{--}$ & $1^{1} P_{1}$ & 6.5954 & 6.5447 & 6.5771 & $6.55-6.82$ & $6.83-6.84$ & 6.420 \\
$1^{--}$ & $1^{5} P_{1}$ & 6.5250 & 6.4676 & 6.4954 & 6.39 & & \\
\hline \hline
\end{tabular}




\section{Conclusion}

This work started as an undergraduate research project to investigate exotic states containing charm quarks [86]. During its development, we dealt with some elements of Quantum Chromodynamics, Quantum Mechanics, numerical methods, charmonium spectroscopy, effective models of heavy quark bound states and the multiquark model of diquarkantidiquark tetraquark.

Even tough we did not dive deep in the deduction of the effective potentials from Relativistic Quantum Field Theory, we managed to trace a consistence trail from a satisfactory reproduction of the charmonium spectrum to the all-charm tetraquark. During that path we learned a lot about these fields of research, and tasted the excitement of keeping track of the recent developments in the area.

Regarding the objectives of the work, we believe to have succeed in our goals. We had the initial proposal of using non-relativistic quarkonium models to study tetraquarks with charm. We faced technical difficulties when for example we confronted the tensor interaction between diquarks. We couldn't find any comprehensive work in the literature that dealt with that in a simple but satisfactory way. So we studied carefully how the tensor works in other contexts and after great discussions with colleagues and professors, we developed a strategy to calculate it with a method that gave results consistent with the results known for two spin $1 / 2$ particles. It might not be the best approach (and we really welcome any critic or alternative), but it made it possible to consider P-wave tetraquark without running away from the problem.

The same effort was made to find a reliable potential and set of parameters. We studied the methods commonly adopted in the simple charmonium models and the experimental progress on its spectroscopy. We were not satisfied with simply picking up any model and set of parameters from literature without probing it and understanding how it worked and the limitations it had. After many preliminary calculations, we concluded that a fitting procedure was our best alternative. Then when the initially adopted model 1 proved to have a few problems in accommodating the experimental data, we looked for a improved version that could do that without changing the whole framework. The model 2 proved itself as a great alternative, and allowed some interesting comparisons between models.

This work could be extended by considering other possibilities, like the color structure of sextet diquarks, different alternatives on how to deal with the string tension and strong coupling constant in the diquark and tetraquark interaction, the inclusion of more terms in the potential like the relativistic correction in the kinetic energy, or even adopting other models to describe the quarks interactions. Also, applying the same model to the case of four bottom quarks is natural extension of this work.

About the all-charm tetraquark, we managed to obtain results compatible with the the literature, and we applied this diquark-antidiquark model to it with some originality, since this detailed analysis of the spin interactions, in particular of the tensor, applied specifically to the tetraquark composed of four charm quarks had never been done before, as it is of our knowledge. The fact that it is relatively simple compared to the 4-body treatment 
and relativistic models allows one to study many of its properties with clarity. Also, it is not just a speculative state, but a great testing probe for the forthcoming higher energy experiments, which makes it one key to understanding the nature of the quickly growing fauna of exotic multiquark candidates. The evolution of our comprehension of these new structures of matter opens new paths towards the understanding of the fundamental forces and particles that compose our reality. 


\section{Appendix A}

\section{Extra Tables: Charmonium}

Here present a few extra results we omitted from the main text.

\section{A.1 Model 1: FIT with 11 input}

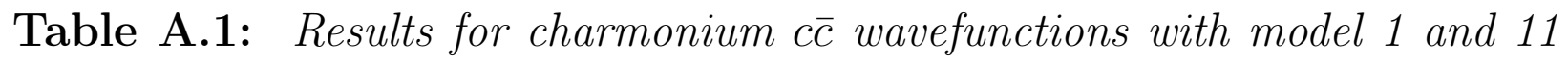
input states. Parameters are $m_{c}=1.4803, \mathrm{GeV}, \alpha_{s}=0.5659, b=0.1451$ $\mathrm{GeV}^{2}$.

\begin{tabular}{crrrr}
\hline \hline$N \ell$ & $M^{(0)}[\mathrm{GeV}]$ & $|R(0)|^{2}\left[\mathrm{GeV}^{3}\right]$ & $\left\langle r^{2}\right\rangle^{1 / 2}[\mathrm{fm}]$ & $\left\langle\frac{v^{2}}{c^{2}}\right\rangle$ \\
\hline $1 S$ & 3.0517 & 1.4909 & 0.395 & 0.288 \\
\hline $1 P$ (c.o.g.) & 3.5230 & 0 & 0.676 & 0.250 \\
\hline $2 S$ & 3.6625 & 0.8037 & 0.846 & 0.299 \\
\hline $1 D$ (c.o.g.) & 3.8062 & 0 & 0.892 & 0.277 \\
\hline $2 P$ (c.o.g.) & 3.9485 & 0 & 1.070 & 0.315 \\
\hline $3 S$ & 4.0726 & 0.6533 & 1.213 & 0.357 \\
\hline $2 D$ (c.o.g.) & 4.1702 & 0 & 1.252 & 0.344 \\
\hline $4 S$ & 4.4138 & 0.5827 & 1.531 & 0.416 \\
\hline \hline
\end{tabular}




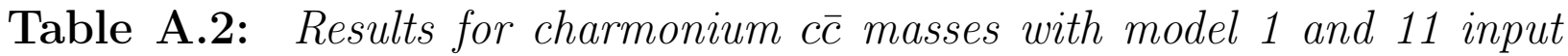
states. Parameters are $m_{c}=1.4803, \mathrm{GeV}, \alpha_{s}=0.5659, b=0.1451 \mathrm{GeV}^{2}$.

\begin{tabular}{crrrrrrrrr}
\hline \hline$N^{2 S+1} \ell_{J}$ & $\langle T\rangle$ & $\left\langle V_{V}^{(0)}\right\rangle$ & $\left\langle V_{S}^{(0)}\right\rangle$ & $E^{(0)}$ & $M^{(0)}[\mathrm{MeV}]$ & $\left\langle V_{S S}^{(1)}\right\rangle$ & $\left\langle V_{L S}^{(1)}\right\rangle$ & $\left\langle V_{T}^{(1)}\right\rangle$ & $M_{1}^{f}[\mathrm{MeV}]$ \\
\hline $1^{1} S_{0}$ & 426.6 & -594.3 & 258.8 & 91.1 & 3051.7 & -257.0 & 0 & 0 & 2795.0 \\
$1^{3} S_{1}$ & 426.6 & -594.3 & 258.8 & 91.1 & 3051.7 & 85.6 & 0 & 0 & 3137.2 \\
\hline $1^{3} P_{0}$ & 370.7 & -274.8 & 466.5 & 562.4 & 3523.0 & 0 & -78.5 & -34.2 & 3410.3 \\
$1^{3} P_{1}$ & 370.7 & -274.8 & 466.5 & 562.4 & 3523.0 & 0 & -39.2 & 17.1 & 3500.9 \\
$1^{1} P_{1}$ (c.o.g.) & 370.7 & -274.8 & 466.5 & 562.4 & 3523.0 & 0 & 0 & 0 & 3523.0 \\
$1^{3} P_{2}$ & 370.7 & -274.8 & 466.5 & 562.4 & 3523.0 & 0 & 39.2 & -3.4 & 3558.8 \\
\hline $2^{1} S_{0}$ & 442.9 & -313.4 & 572.5 & 701.9 & 3662.5 & -138.0 & 0 & 0 & 3524.2 \\
$2^{3} S_{1}$ & 442.9 & -313.4 & 572.5 & 701.9 & 3662.5 & 46.1 & 0 & 0 & 3708.7 \\
\hline $1^{3} D_{1}$ & 410.5 & -192.9 & 628.1 & 845.6 & 3806.2 & 0 & -13.0 & -4.3 & 3789.0 \\
$1^{3} D_{2}$ & 410.5 & -192.9 & 628.1 & 845.6 & 3806.2 & 0 & -4.3 & 4.3 & 3806.2 \\
$1^{1} D_{2}$ (c.o.g.) & 410.5 & -192.9 & 628.1 & 845.6 & 3806.2 & 0 & 0 & 0 & 3806.2 \\
$1^{3} D_{3}$ & 410.5 & -192.9 & 628.1 & 845.6 & 3806.2 & 0 & 8.7 & -1.2 & 3813.7 \\
\hline $2^{3} P_{0}$ & 466.7 & -206.0 & 727.3 & 987.9 & 3948.5 & 0 & -71.9 & -30.0 & 3846.6 \\
$2^{3} P_{1}$ & 466.7 & -206.0 & 727.3 & 987.9 & 3948.5 & 0 & -36.0 & 15.0 & 3927.6 \\
$2^{1} P_{1}$ (c.o.g.) & 466.7 & -206.0 & 727.3 & 987.9 & 3948.5 & 0 & 0 & 0 & 3948.5 \\
$2^{3} P_{2}$ & 466.7 & -206.0 & 727.3 & 987.9 & 3948.5 & 0 & 36.0 & -3.0 & 3981.5 \\
\hline $3^{1} S_{0}$ & 528.4 & -236.7 & 820.2 & 1112.0 & 4072.6 & -112.0 & 0 & 0 & 3960.1 \\
$3^{3} S_{1}$ & 528.4 & -236.7 & 820.2 & 1112.0 & 4072.6 & 37.5 & 0 & 0 & 4110.1 \\
\hline $2^{3} D_{1}$ & 509.8 & -160.0 & 859.7 & 1209.6 & 4170.2 & 0 & -15.4 & -4.1 & 4150.7 \\
$2^{3} D_{2}$ & 509.8 & -160.0 & 859.7 & 1209.6 & 4170.2 & 0 & -5.1 & 4.1 & 4169.1 \\
$2^{1} D_{2}$ (c.o.g.) & 509.8 & -160.0 & 859.7 & 1209.6 & 4170.2 & 0 & 0 & 0 & 4170.2 \\
$2^{3} D_{3}$ & 509.8 & -160.0 & 859.7 & 1209.6 & 4170.2 & 0 & 10.3 & -1.2 & 4179.3 \\
\hline $4^{1} S_{0}$ & 616.1 & -197.6 & 1035.0 & 1453.2 & 4413.8 & -100.0 & 0 & 0 & 4313.5 \\
$4^{3} S_{1}$ & 616.1 & -197.6 & 1035.0 & 1453.2 & 4413.8 & 33.4 & 0 & 0 & 4447.2 \\
\hline \hline
\end{tabular}




\section{A.2 Model 2: FIT with 11 input}

Table A.3: Results for charmonium c $\bar{c}$ wavefunctions with model 2 and 11 input states. Parameters are $m_{c}=1.4685 \mathrm{GeV}, \alpha_{s}=0.5285, b=0.1458$ $\mathrm{GeV}^{2}, \sigma=1.1779 \mathrm{GeV}$.

\begin{tabular}{crrrr}
\hline \hline$N^{2 S+1} \ell$ & $M^{(0)}[\mathrm{GeV}]$ & $|R(0)|^{2}\left[\mathrm{GeV}^{3}\right]$ & $\left\langle r^{2}\right\rangle^{1 / 2}[\mathrm{fm}]$ & $\left\langle\frac{v^{2}}{c^{2}}\right\rangle$ \\
\hline $1^{1} S$ & 2.9816 & 1.7151 & 0.366 & 0.349 \\
$1^{3} S$ & 3.0900 & 1.2956 & 0.415 & 0.258 \\
\hline $1^{1} P$ & 3.5156 & 0 & 0.674 & 0.254 \\
$1^{3} P$ (c.o.g.) & 3.5246 & 0 & 0.686 & 0.243 \\
\hline $2^{1} S$ & 3.6303 & 0.7961 & 0.833 & 0.304 \\
$2^{3} S$ & 3.6718 & 0.7482 & 0.863 & 0.289 \\
\hline $1^{1} D$ & 3.7994 & 0 & 0.899 & 0.274 \\
$1^{3} D$ (c.o.g.) & 3.8001 & 0 & 0.900 & 0.272 \\
\hline $2^{1} P$ & 3.9336 & 0 & 1.070 & 0.318 \\
$2^{3} P$ (c.o.g.) & 3.9432 & 0 & 1.082 & 0.308 \\
\hline $3^{1} S$ & 4.0432 & 0.6353 & 1.208 & 0.356 \\
$3^{3} S$ & 4.0716 & 0.6174 & 1.229 & 0.349 \\
\hline $2^{1} D$ & 4.1576 & 0 & 1.260 & 0.341 \\
$2^{3} D$ (c.o.g.) & 4.1589 & 0 & 1.262 & 0.339 \\
\hline $4^{1} S$ & 4.3837 & 0.5634 & 1.531 & 0.413 \\
$4^{3} S$ & 4.4061 & 0.5543 & 1.548 & 0.409 \\
\hline \hline
\end{tabular}




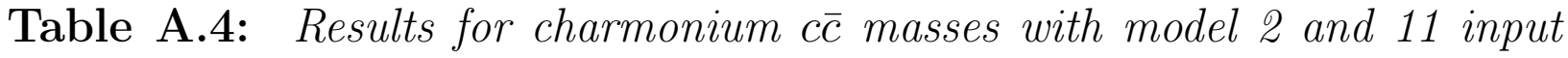
states. Parameters are $m_{c}=1.4685 \mathrm{GeV}, \alpha_{s}=0.5285, b=0.1458 \mathrm{GeV}^{2}$, $\sigma=1.1779 \mathrm{GeV}$.

\begin{tabular}{crrrrrrrrr}
\hline \hline$N^{2 S+1} \ell_{J}$ & $\langle T\rangle$ & $\left\langle V_{V}^{(0)}\right\rangle$ & $\left\langle V_{S}^{(0)}\right\rangle$ & $\left\langle V_{S S}^{(0)}\right\rangle$ & $E^{(0)}$ & $M^{(0)}[\mathrm{MeV}]$ & $\left\langle V_{L S}^{(1)}\right\rangle$ & $\left\langle V_{T}^{(1)}\right\rangle$ & $M_{2}^{f}[\mathrm{MeV}]$ \\
\hline $1^{1} S_{0}$ & 515.7 & -632.5 & 233.5 & -93.9 & 22.8 & 2981.6 & 0 & 0 & 2981.6 \\
$1^{3} S_{1}$ & 381.4 & -540.7 & 267.4 & 23.1 & 131.2 & 3090.0 & 0 & 0 & 3090.0 \\
\hline $1^{3} P_{0}$ & 358.8 & -260.4 & 465.3 & 2.1 & 565.8 & 3524.6 & -69.3 & -30.9 & 3424.4 \\
$1^{3} P_{1}$ & 358.8 & -260.4 & 465.3 & 2.1 & 565.8 & 3524.6 & -34.6 & 15.4 & 3505.4 \\
$1^{1} P_{1}$ & 375.7 & -267.7 & 456.2 & -7.4 & 556.8 & 3515.6 & 0 & 0 & 3515.6 \\
$1^{3} P_{2}$ & 358.8 & -260.4 & 465.3 & 2.1 & 565.8 & 3524.6 & 34.6 & -3.1 & 3556.1 \\
\hline $2^{1} S_{0}$ & 449.6 & -302.7 & 555.6 & -30.9 & 671.5 & 3630.3 & 0 & 0 & 3630.3 \\
$2^{3} S_{1}$ & 427.1 & -297.1 & 572.7 & 10.3 & 713.0 & 3671.8 & 0 & 0 & 3671.8 \\
\hline $1^{3} D_{1}$ & 402.9 & -184.4 & 622.6 & 0.2 & 841.3 & 3800.1 & -11.2 & -4.0 & 3785.0 \\
$1^{3} D_{2}$ & 402.9 & -184.4 & 622.6 & 0.2 & 841.3 & 3800.1 & -3.7 & 4.0 & 3800.4 \\
$1^{1} D_{2}$ & 404.7 & -184.9 & 621.4 & -0.6 & 840.6 & 3799.4 & 0 & 0 & 3799.4 \\
$1^{3} D_{3}$ & 402.9 & -184.4 & 622.6 & 0.2 & 841.3 & 3800.1 & 7.4 & -1.1 & 3806.4 \\
\hline $2^{3} P_{0}$ & 455.3 & -195.7 & 722.6 & 2.2 & 984.4 & 3943.2 & -63.8 & -27.1 & 3852.3 \\
$2^{3} P_{1}$ & 455.3 & -195.7 & 722.6 & 2.2 & 984.4 & 3943.2 & -31.9 & 13.6 & 3924.9 \\
$2^{1} P_{1}$ & 470.1 & -200.9 & 713.3 & -7.8 & 974.8 & 3933.6 & 0 & 0 & 3933.6 \\
$2^{3} P_{2}$ & 455.3 & -195.7 & 722.6 & 2.2 & 984.4 & 3943.2 & 31.9 & -2.7 & 3972.4 \\
\hline $3^{1} S_{0}$ & 527.0 & -225.9 & 804.0 & -20.7 & 1084.4 & 4043.2 & 0 & 0 & 4043.2 \\
$3^{3} S_{1}$ & 516.1 & -226.1 & 815.6 & 7.2 & 1112.8 & 4071.6 & 0 & 0 & 4071.6 \\
\hline $2^{3} D_{1}$ & 501.3 & -152.9 & 851.4 & 0.3 & 1200.1 & 4158.9 & -13.6 & -3.8 & 4141.5 \\
$2^{3} D_{2}$ & 501.3 & -152.9 & 851.4 & 0.3 & 1200.1 & 4158.9 & -4.5 & 3.8 & 4158.2 \\
$2^{1} D_{2}$ & 504.1 & -153.7 & 849.4 & -1.0 & 1198.8 & 4157.6 & 0.0 & 0.0 & 4157.6 \\
$2^{3} D_{3}$ & 501.3 & -152.9 & 851.4 & 0.3 & 1200.1 & 4158.9 & 9.1 & -1.1 & 4166.9 \\
\hline $4^{1} S_{0}$ & 611.6 & -188.0 & 1018.0 & -16.3 & 1424.9 & 4383.7 & 0.0 & 0.0 & 4383.7 \\
$4^{3} S_{1}$ & 604.5 & -189.4 & 1026.0 & 5.8 & 1447.3 & 4406.1 & 0.0 & 0.0 & 4406.1 \\
\hline \hline
\end{tabular}




\section{Bibliography}

[1] D. Griffiths, Introduction to Elementary Particles, Second Revised Edition, Wiley-VCH (2008). 1, 15, 24, 58

[2] R. L. Jaffe, Multi-Quark Hadrons. 1. The Phenomenology of (2 Quark 2 anti-Quark) Mesons, Phys. Rev. D 15, 267 (1977). 3

[3] R. L. Jaffe, Multi-Quark Hadrons. 2. Methods, Phys. Rev. D 15, 281 (1977). 3

[4] J.-P. Ader, J.-M. Richard, P. Taxil, Do Narrow Heavy Multi - Quark States Exist?, Phys. Rev. D 25, 2370 (1982). 3, 5, 83, 93

[5] J. D. Weinstein, N. Isgur, The q q anti-q anti-q System in a Potential Model, Phys. Rev. D 27, 588 (1983). 3

[6] J. D. Weinstein, N. Isgur, K anti-K Molecules, Phys. Rev. D 41, 2236 (1990). 3

[7] S. L. Olsen, A New Hadron Spectroscopy, Front. Phys. 10, 101401 (2015). 4

[8] A. Esposito, A. L. Guerrieri, F. Piccinini, A. Pilloni, A. D. Polosa, Four-Quark Hadrons: an Updated Review, Int. J. Mod. Phys. A 30, 1530002 (2014). 4, 5

[9] R. Aaij et al., LHCb Collaboration, Observation of the resonant character of the Z(4430)state, Phys. Rev. Lett. 112, no. 22, 222002 (2014). 4

[10] M. Nielsen, F. S. Navarra, Charged Exotic Charmonium States, Mod. Phys. Lett. A 29, 1430005 (2014). 4

[11] N. Brambilla et al., Heavy Quarkonium Working Group, Heavy quarkonium: progress, puzzles, and opportunities, Eur. Phys. J. C 71, 1534 (2011). 4

[12] M. Nielsen, F. S. Navarra, S. H. Lee, New Charmonium States in QCD Sum Rules: a Concise Review, Phys. Rept. 497, 41 (2010). 4

[13] R. L. Jaffe, F. Willczek, Diquarks and exotic spectroscopy, Phys. Rev. Lett. 91, 232003 (2003). 4

[14] M. B. Voloshin, L. B. Okun, Hydronic molecules and the charmonium atom, [Pisma Zh. Eksp. Teor. Fiz. 23, 369 (1976)], JETP Lett. 23, 333 (1976). 4

[15] A. De Rujula, H. Georgi, S. L. Glashow, Molecular Charmonium: A New Spectroscopy?, Phys. Rev. Lett. 38, 317 (1977). 4

[16] A. Le Yaouanc, L. Oliver, O. Pene, J. C. Raynal, Strong Decays of psi-prime-prime (4.028) as a Radial Excitation of Charmonium, Phys. Lett. B 71, 397 (1977). 4

[17] A. Le Yaouanc, L. Oliver, O. Pene, J. C. Raynal, Why is psi-prime-prime-prime (4.414) So Narrow?, Phys. Lett. B 72, 57 (1977). 4 
[18] N. A. Tornqvist, From the deuteron to deusons, an analysis of deuteron - like meson meson bound states, Z. Phys. C 61, 525 (1994). 4

[19] T. E. O. Ericson, G. Karl, Strength of pion exchange in hadronic molecules, Phys. Lett. B 309, 426 (1993). 4

[20] A. V. Manohar, M. B. Wise, Exotic $Q Q$ anti-q anti-q states in $Q C D$, Nucl. Phys. B 399, 17 (1993). 4

[21] E. S. Swanson, The New heavy mesons: A Status report, Phys. Rept. 429, 243 (2006). 4

[22] J. Vijande, A. Valcarce, J. M. Richard, N. Barnea, Four-quark stability, Few Body Syst. 45, 99 (2009). 4

[23] L. Maiani, F. Piccinini, A. D. Polosa, V. Riquer, Diquark-antidiquarks with hidden or open charm and the nature of X(3872), Phys. Rev. D 71, 014028 (2005). 4, 32

[24] L. Maiani, A. D. Polosa, V. Riquer, The Charged Z(4433): Towards a New Spectroscopy, arXiv:0708.3997 [hep-ph] (2007). 4

[25] L. Maiani, A. D. Polosa, V. Riquer, The charged Z(4430) in the diquark-antidiquark picture, New J. Phys. 10, 073004 (2008). 4

[26] L. Maiani, F. Piccinini, A. D. Polosa, V. Riquer, The Z(4430) and a New Paradigm for Spin Interactions in Tetraquarks, Phys. Rev. D 89, 114010 (2014). 4, 32

[27] A. Ali, L. Maiani, A. D. Polosa, V. Riquer, Hidden-Beauty Charged Tetraquarks and Heavy Quark Spin Conservation, Phys. Rev. D 91, no. 1, 017502 (2015). 4

[28] F. S. Navarra, M. Nielsen, J. M. Richard, Exotic Charmonium and Bottomonium-like Resonances, J. Phys. Conf. Ser. 348, 012007 (2012). 4

[29] F.S. Navarra, M. Nielsen, S. H. Lee, QCD sum rules study of $Q Q$ - anti-u anti-d mesons, Phys. Lett. B 649, 166 (2007). 4

[30] Y. Iwasaki, A Possible Model for New Resonances-Exotics and Hidden Charm, Prog. Theor. Phys. 54, 492 (1975) 5

[31] Y. Iwasaki, 'How to Find eta (C) and a Possible State Charm anti-Charm Charm anti-Charm,, Phys. Rev. D 16, 220 (1977). 5

[32] K. T. Chao, The (c c) - (anti-c anti-c) (Diquark - anti-Diquark) States in e+e-Annihilation, Z. Phys. C 7, 317 (1981). 5, 32, 94

[33] J. L. Ballot, J.-M. Richard, Four Quark States In Additive Potentials, Phys. Lett. B 123, 449 (1983). 5

[34] H. J. Lipkin, A Model Independent Approach To Multi - Quark Bound States, Phys. Lett. B 172, 242 (1986). 5

[35] L. Heller, J. A. Tjon, On Bound States of Heavy $Q^{2} \bar{Q}^{2}$ Systems, Phys. Rev. D 32, 755 (1985). 5

[36] L. Heller, J. A. Tjon, On the Existence of Stable Dimesons, Phys. Rev. D 35, 969 (1987). 5

[37] B. Silvestre-Brac, Systematics of $Q^{* * 2}$ (anti-Q**2) systems with a chromomagnetic interaction, Phys. Rev. D 46, 2179 (1992). 5 
[38] B. Silvestre-Brac, C. Semay, Systematics of $L=0$ q-2 anti-q-2 systems, Z. Phys. C 57, 273 (1993). 5

[39] B. Silvestre-Brac, C. Semay, Spectrum and decay properties of diquonia, Z. Phys. C 59,457 (1993). 5

[40] B. Silvestre-Brac, C. Semay, Diquonia and potential models, Z. Phys. C 61, 271 (1994). 5

[41] R. J. Lloyd, J. P. Vary, All charm tetraquarks, Phys. Rev. D 70, 014009 (2004). 5

[42] N. Barnea, J. Vijande, A. Valcarce, Four-quark spectroscopy within the hyperspherical formalism, Phys. Rev. D 73, 054004 (2006). 5

[43] W. Heupel, G. Eichmann, C. S. Fischer, Tetraquark Bound States in a Bethe-Salpeter Approach, Phys. Lett. B 718, 545 (2012). 5

[44] T. W. Chiu et al. [TWQCD Collaboration], Y(4260) on the lattice, Phys. Rev. D 73, 094510 (2006) 5,94

[45] M. Wagner, A. Abdel-Rehim, C. Alexandrou, M. Dalla Brida, M. Gravina, G. Koutsou, L. Scorzato, C. Urbach, Investigation of light and heavy tetraquark candidates using lattice QCD, J. Phys. Conf. Ser. 503, 012031 (2014). 5

[46] P. Bicudo, K. Cichy, A. Peters, B. Wagenbach, M. Wagner, Evidence for the existence of $u d \bar{b} \bar{b}$ and the non-existence of $s s \bar{b} \bar{b}$ and $c c \bar{b} \bar{b}$ tetraquarks from lattice $Q C D$, Phys. Rev. D 92, 014507 (2015). 5

[47] A. V. Berezhnoy, A. K. Likhoded, A. V. Luchinsky, A. A. Novoselov, Double J/psi-meson Production at LHC and 4c-tetraquark state, Phys. Rev. D 84, 094023 (2011). 5, 94

[48] A. V. Berezhnoy, A. V. Luchinsky, A. A. Novoselov, Heavy tetraquarks production at the LHC, Phys. Rev. D 86, 034004 (2012). 5, 32, 94

[49] F. Carvalho, E. R. Cazaroto, V. P. Gonçalves, F. S. Navarra, Tetraquark Production in Double Parton Scattering, Phys. Rev. D 93, no. 3, 034004 (2016). 6

[50] J. Wu, Y. R. Liu, K. Chen, X. Liu and S. L. Zhu, Heavy-flavored tetraquark states with the $Q Q \bar{Q} \bar{Q}$ configuration, arXiv:1605.01134 [hep-ph]. 6, 17, 94

[51] W. Chen, H. X. Chen, X. Liu, T. G. Steele, S. L. Zhu, Hunting for exotic doubly hiddencharm/bottom tetraquark states, arXiv:1605.01647 [hep-ph] (2016). 6, 94

[52] K. A. Olive et al., Particle Data Group Collaboration, Review of Particle Physics, Chin. Phys. C 38, 090001 (2014); 2015 update on charmonium: http://pdg.lbl.gov/2015/tables/rpp2015tab-mesons-c-cbar.pdf $6,25,58,60,64,69,70,74,75$

[53] F. K. Guo, U. G. Meissner, Where is the $\chi_{c 0}(2 P)$ ?, Phys. Rev. D 86, 091501 (2012). 6, 75

[54] S. L. Olsen, Is the $X(3915)$ the $\chi_{c 0}(2 P)$ ?, Phys. Rev. D 91, no. 5, 057501 (2015). 6, 75

[55] R. F. Lebed, A. D. Polosa, $\chi_{c 0}(3915)$ As the Lightest c $\bar{c} s \bar{s}$ State, arXiv:1602.08421 [hep-ph] (2016). 6, 75

[56] J. Zhang, Experimental overview of charmonium spectroscopy, arXiv:1311.3370 [hep-ex] (2013). 6, 70, 71

[57] E. Eichten, K. Gottfried, T. Konoshita, K. D. Lane, T. M. Yan, Charmonium: The model, Phys. Rev. D 17, 3090 (1978); Erratum: Phys. Rev. D 21, 313 (1980). 9 
[58] E. Eichten, K. Gottfried, T. Konoshita, K. D. Lane, T. M. Yan, Charmonium: Comparison with experiment, Phys. Rev. D 21, 203 (1980). 9, 63, 65

[59] W. Lucha, F. F. Schoberl, Effective potential models for hadrons, arXiv:hep-ph/9601263 (1995). 9, 12, 13, 14, 15, 64

[60] W. Lucha, F. F. Schoberl, D. Gromes, Bound States of Quarks, Phys. Rept. 200, 127-240 (1991). 9, 12, 13, 14, 15, 27, 38, 64

[61] W. Lucha, F. F. Schöberl, Solving the Schrödinger equation for bound states with Mathematica 3.0, Int. J. Mod. Phys. C 10, 607 (1999). 11, 26

[62] M. De Sanctis, P. Quintero, Charmonium spectrum with a generalized Fermi-Breit equation, Eur. Phys. J. A 46213 (2010). 11, 57, 59, 63, 65, 78

[63] J. L. Domenech-Garret, M. A. Sanchis-Lozano, QQ-onia package: A Numerical solution to the Schrodinger radial equation for heavy quarkonium, Comput. Phys. Commun. 180, 768 (2009). 12, 25, 28, 55

[64] M. B. Voloshin, Charmonium, Prog. Part. Nucl. Phys. 61, 455 (2008). 12, 25

[65] J. Eiglsperger, Quarkonium Spectroscopy: Beyond One-Gluon Exchange, arXiv:0707.1269 [hep-ph] (2007). 12, 13, 15, 64

[66] T. Barnes, Charmonium at BES and CLEO-c, arXiv:hep-ph/0406327. 13, 14, 28, 57, 64, 67, $69,73,74$

[67] T. Barnes, S. Godfrey, E. S. Swanson, Higher charmonia, Phys. Rev. D 72, 054026 (2005). $13,14,25,28,57,59,64,65,67,69,70,73,74,75$

[68] S. Godfrey, N. Isgur, Mesons in a Relativized Quark Model with Chromodynamics, Phys. Rev. D 32, 189 (1985). 13, 25

[69] D. M. Brink and F. Stancu, Role of hidden color states in 2 q 2 anti-q systems, Phys. Rev. D 49, 4665 (1994). 13, 30

[70] T. Muta, Foundations of Quantum Chromodynamics: An Introduction to Perturbative Methods in Gauge Theories, World Scientific Lecture Notes in Physics: Vol. 78, 3rd edition (2010). 17

[71] Fayyazuddin, Riazuddin, A Modern Introduction to Particle Physics, World Scientific Publishing, 3rd edition (2011). 17

[72] F. Stancu, Group theory in subnuclear physics, Oxford Stud. Nucl. Phys. 19 (1996). 17, 30

[73] D. M. Brink, F. Stancu, Tetraquarks with heavy flavors, Phys. Rev. D 57, 6778 (1998). 17, 30,55

[74] W. Park, S. H. Lee, Color spin wave functions of heavy tetraquark states, Nucl. Phys. A 925, 161 (2014). 17, 30, 55

[75] A. Messiah, Quantum Mechanics, Vol. II, North Holland, John Wiley \& Sons (1966). 38

[76] L. Lovitch, S. Rosati, Direct Numerical Integration of the Two-Nucleon Schrodinger Equation with Tensor Forces, Phys. Rev. 140, B877 (1965). 38

[77] M. Naghdi, Nucleon-Nucleon Interaction: A Typical/Concise Review, Phys. Part. Nucl. 45, 924 (2014). 38 
[78] D. Griffiths, Introduction to Quantum Mechanics, Second Edition, Pearson Prentice Hall (2005). 38

[79] H. A. Bethe, E. E. Salpether, Quantum Mechanics of atoms of one- and two-electrons, Springer (1957). 38, 39

[80] C. Cohen-Tannoudji, B. Diu, F. Laloe, Quantum Mechanics, Vol. 2, Wiley-VHC (1978). 41

[81] Anthony Stone's Wigner coefficient calculator, http://www-stone.ch.cam.ac.uk/wigner.shtml 44

[82] V. V. Kiselev, A. K. Likhoded, O. N. Pakhomova, V. A. Saleev, Mass spectra of doubly heavy $\Omega_{Q Q^{\prime}}$ baryons, Phys. Rev. D 66, 034030 (2002). 77, 83, 94

[83] C. Gutierrez, M. De Sanctis, A study of a relativistic quark-diquark model for the nucleon, Eur. Phys. J. A 50, no. 11, 169 (2014). 77

[84] D. Ebert, R. N. Faustov, V. O. Galkin, W. Lucha, Masses of tetraquarks with two heavy quarks in the relativistic quark model, Phys. Rev. D 76, 114015 (2007) 78, 83

[85] Q. F. Lü, Y. B. Dong, Masses of open charm and bottom tetraquark states in relativized quark model, arXiv:1603.06417 [hep-ph] (2016). 78

[86] V. R. Debastiani, F. S. Navarra, Charm tetraquarks in a non-relativistic quark model, J.Phys.Conf.Ser. 630 (2015) 012047, J. Phys. Conf. Ser. 630, no. 1, 012047 (2015). 95 